\title{
Nonequilibrium steady states of ideal bosonic and fermionic quantum gases
}

\author{
Daniel Vorberg, ${ }^{1, *}$ Waltraut Wustmann, ${ }^{1,2}$ Henning Schomerus, ${ }^{1,3}$ Roland Ketzmerick, ${ }^{1,2}$ and André Eckardt ${ }^{1, \dagger}$ \\ ${ }^{1}$ Max-Planck-Institut für Physik komplexer Systeme, Nöthnitzer Strasse 38, 01187 Dresden, Germany \\ ${ }^{2}$ Technische Universität Dresden, Institut für Theoretische Physik and Center for Dynamics, 01062 Dresden, Germany \\ ${ }^{3}$ Department of Physics, Lancaster University, Lancaster LA1 4YB, United Kingdom
}

(Received 12 August 2015; published 9 December 2015)

\begin{abstract}
We investigate nonequilibrium steady states of driven-dissipative ideal quantum gases of both bosons and fermions. We focus on systems of sharp particle number that are driven out of equilibrium either by the coupling to several heat baths of different temperature or by time-periodic driving in combination with the coupling to a heat bath. Within the framework of (Floquet-)Born-Markov theory, several analytical and numerical methods are described in detail. This includes a mean-field theory in terms of occupation numbers, an augmented mean-field theory taking into account also nontrivial two-particle correlations, and quantum-jump-type Monte Carlo simulations. For the case of the ideal Fermi gas, these methods are applied to simple lattice models and the possibility of achieving exotic states via bath engineering is pointed out. The largest part of this work is devoted to bosonic quantum gases and the phenomenon of Bose selection, a nonequilibrium generalization of Bose condensation, where multiple single-particle states are selected to acquire a large occupation [Phys. Rev. Lett. 111, 240405 (2013)]. In this context, among others, we provide a theory for transitions where the set of selected states changes, describe an efficient algorithm for finding the set of selected states, investigate beyond-mean-field effects, and identify the dominant mechanisms for heat transport in the Bose-selected state.
\end{abstract}

DOI: 10.1103/PhysRevE.92.062119

PACS number(s): 05.30.Jp, 05.70.Ln, 67.10.Ba, 67.85.Jk

\section{INTRODUCTION}

There is a huge current interest in nonequilibrium phenomena of many-body systems beyond the hydrodynamic description of systems retaining approximate local equilibrium. Recent work concerns several paradigmatic scenarios, like the dynamics away from equilibrium in response to a slow or an abrupt parameter variation [1-3], the possible relaxation towards equilibrium [2,3] versus many-body localization [4,5], and the control of many-body physics by means of strong periodic forcing [6-11]. Also the possibility to achieve transient light-induced superconductivity above the equilibrium critical temperature attracted enormous interest [12].

Another fundamental scenario of many-body dynamics consists of driven-dissipative quantum systems and their nonequilibrium steady states [13-22]. These include, for example, time-periodically driven open many-body systems [23-27] and photonic many-body systems [28-33]. In contrast to equilibrium states, which depend on a few thermodynamic parameters like temperature and chemical potential only, such nonequilibrium steady states depend on the very details of the environment. On the one hand, this makes their theoretical treatment challenging. On the other hand, it offers also interesting opportunities to engineer the state and the properties of a many-body system beyond the constraints of thermal equilibrium in a robust and controlled fashion.

In this context, it was recently pointed out that already an ideal Bose gas of $N$ particles can exhibit intriguing behavior, when it is driven into a steady state far from equilibrium, e.g., by coupling it to two heat baths of different temperature or by time-periodic driving in the presence of a heat bath (see Fig. 1). In the quantum degenerate regime of

\footnotetext{
*dv@pks.mpg.de

†eckardt@pks.mpg.de
}

large densities, the Bose gas undergoes a generalized form of Bose condensation, where multiple single-particle states can be selected to acquire large occupations [23]. Namely, the single-particle states unambiguously separate into two groups: one that is called Bose selected, whose occupations increase linearly when the total particle number is increased at fixed system size, and another one whose occupations saturate. This phenomenon is a consequence of the bosonic quantum statistics. It includes standard Bose condensation into a single quantum state, fragmented Bose condensation into a small number of single-particle states, each acquiring a macroscopic occupation, and the case where a fraction of all single-particle states acquires large, but individually nonextensive occupations. The properties of the system, like its coherence or its heat conductivity, sensitively depend on which of these scenarios occurs.

The physics of driven-dissipative ideal Bose gases is intimately related also to collective effects in classical systems and processes, where bunching phenomena have been identified as analog of Bose condensation. This includes the dynamics of networks and economic models [34,35], classical transport and traffic [36-44], chemical reactions [45], as well as population dynamics and evolutionary game theory [46]. These connections have recently been discussed by Knebel et al. [47].

In this paper, we investigate nonequilibrium steady states of driven-dissipative ideal quantum gases of both bosons and fermions. We focus on systems of sharp particle number that exchange energy with the environment. These quantum gases are driven out of equilibrium either by the coupling to several heat baths of different temperature or by time-periodic driving in combination with the coupling to a heat bath (see Fig. 1). We treat the problem using (Floquet-)Born-Markov theory [48-52], which is valid in the limit of weak system-bath coupling. In Sec. II this theoretical framework is reviewed and applied to the problem of the ideal quantum gas. Moreover, 


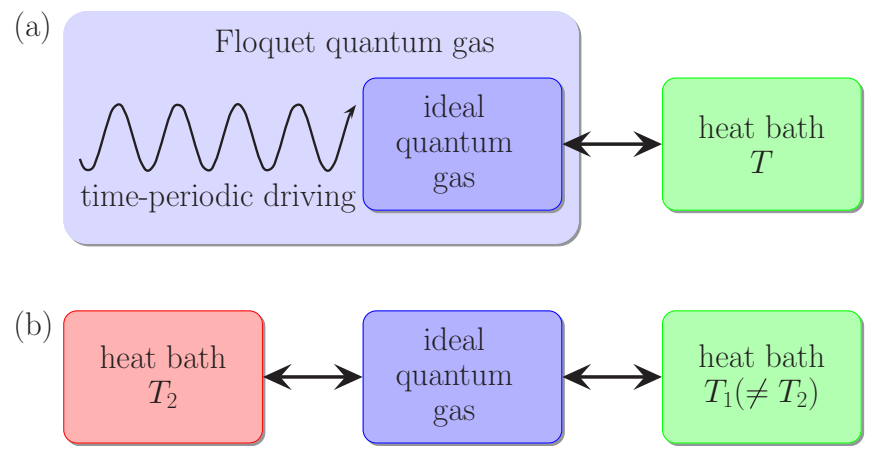

FIG. 1. (Color online) Two paradigmatic examples of drivendissipative ideal quantum gases possessing nonequilibrium steady states. (a) Periodically driven system weakly coupled to a heat bath. (b) Autonomous system weakly coupled to two heat baths of different temperature.

several model systems are introduced. In order to treat the resulting many-body master equation, we then describe analytical and numerical methods for computing the steady state (Sec. III). This includes a standard mean-field description in terms of single-particle occupation numbers. We, moreover, derive an augmented mean-field theory taking into account also nontrivial two-particle correlations and explain how to apply quantum-jump-type Monte Carlo simulations to the problem. These methods are then applied to both the ideal Bose gas (Sec. IV) and the ideal Fermi gas (Sec. V).

Our treatment of the fermionic case in Sec. V is rather brief and demonstrates the application of our theory to simple lattice models and the possibility to achieve exotic states via bath engineering. These results can be relevant, e.g., for the problem of realizing Floquet topological insulators with periodically forced electronic systems (graphene [7] or semiconductor heterostructures [53]).

The largest part of this paper is devoted to bosonic quantum gases and the phenomenon of Bose selection discussed in Sec. IV. Here we first review equilibrium Bose condensation (Sec. IV A) and Bose selection in nonequilibrium steady states (Secs. IV B to IVE give a detailed discussion of the results of Ref. [23]). After that, we derive a theory for transitions where the set of selected states changes (Sec. IV F), present an efficient algorithm for finding the set of selected states ( $\mathrm{Sec}$. IV G), discuss the possibility of approaching a preasymptotic state at intermediate densities before the true asymptotic state is reached at large densities (Sec. IV H), investigate the properties of systems described by non-fully-connected rate matrices (Sec. IV I), study the role of fluctuations and beyond mean-field effects (Sec. IV J), and identify the dominant mechanisms for heat transport in the Bose-selected state Sec. IV K.

\section{GENERAL FRAMEWORK AND MODELS}

In this section we set up the master equations for an ideal quantum gas of $N$ indistinguishable, noninteracting particles, weakly coupled to one or several heat baths. We cover both the case of an autonomous system with time-independent Hamiltonian $\hat{H}$ and the case of a Floquet system with time-periodic Hamiltonian $\hat{H}(t)=\hat{H}(t+\tau)$. This captures the nonequilibrium situations depicted in Fig. 1. In the case of the periodically driven system, we encounter the Floquet states $\left|\phi_{i}(t)\right\rangle=e^{-i \varepsilon_{i} t / \hbar}|i(t)\rangle$, which are quasistationary (i.e., timeperiodic) solutions of the dynamics generated by $\hat{H}(t)$ [54-56]. Here $|i(t)\rangle=|i(t+\tau)\rangle$ denotes time-periodic Floquet modes, while $\varepsilon_{i}$ are the quasienergies, which are defined modulo the energy quantum $\hbar \omega$ with angular driving frequency $\omega=2 \pi / \tau$. We start with the single-particle equations. In Sec. II B, we then generalize to the many-body case.

\section{A. Single-particle master equation}

We consider the time evolution of the density operator $\hat{\rho}$ in a single-particle system. In the weak-coupling limit, where the full rotating-wave approximation is valid, this time evolution is governed by a master equation of Lindblad type [48], which in the interaction picture reads

$$
\frac{d \hat{\rho}(t)}{d t}=\mathcal{D}[\hat{\rho}]=\sum_{i, j} R_{i j}\left[\hat{L}_{i j} \hat{\rho}(t) \hat{L}_{i j}^{\dagger}-\frac{1}{2}\left\{\hat{\rho}(t), \hat{L}_{i j}^{\dagger} \hat{L}_{i j}\right\}\right] .
$$

Here $\{A, B\}=A B+B A$ denotes the anticommutator. The indices enumerate the energy eigenstates of the autonomous system or the Floquet states of the periodically driven system. In practice, we restrict the number $M$ of participating single-particle states to be finite. The dissipation causes transitions from eigenstate $|j\rangle$ to eigenstate $|i\rangle$ according to the jump operator $\hat{L}_{i j}=|i\rangle\langle j|$, where $R_{i j}$ is the corresponding transition rate. This description is valid in the weak-coupling limit, where the level broadening $\hbar R_{i j}$ due to the transitions is much smaller than the (typical) energy separation of neighboring (quasi)energy levels in the spectrum of the system. The characteristic time scale $\tau_{S}$ of the unitary dynamics is then much smaller than the time scale $\tau_{R}$ of the dissipative relaxation, $\tau_{S} \ll \tau_{R}$, which makes it possible to employ the full rotating-wave approximation leading to Eq. (1) [49-52].

Since the resulting Lindblad equation (1) is diagonal in the basis of states $|i\rangle$, the dynamics of the occupation probabilities $p_{i}=\langle i|\hat{\rho}| i\rangle$ decouples from the off-diagonal elements of the density operator, which decay as one approaches the steady state. The dynamics of the diagonal elements are described by the Pauli master equation

$$
\dot{p}_{i}(t)=\sum_{j}\left[R_{i j} p_{j}(t)-R_{j i} p_{i}(t)\right]
$$

The terms of the sum correspond to the net probability flux from states $j$ to state $i$. The uniqueness of the steady state $\hat{\rho}=\sum_{i} p_{i}|i\rangle\langle i|$, obtained by requiring $\dot{p}_{i}=0$, is guaranteed by the Frobenius-Perron theorem, which holds if every state is connected with all the other states by a sequence of transitions with nonvanishing rates [57].

For the weak coupling to the environment considered here, the rates $R_{i j}$ in Eq. (2) can, in general, be determined in the Born-Markov (Floquet-Born-Markov) approximation for autonomous (time-periodically driven) systems. We consider that a bath is given by a collection of harmonic oscillators $\alpha$ with angular frequency $\omega_{\alpha}$ and annihilation operator $\hat{b}_{\alpha}$, described by the bath Hamiltonian $\hat{H}_{B}=\sum_{\alpha} \hbar \omega_{\alpha} \hat{b}_{\alpha}^{\dagger} \hat{b}_{\alpha}$. The bath is in thermal equilibrium with temperature $T$ and coupled 
to the system via the Hamiltonian $\hat{H}_{S B}=\hat{v} \sum_{\alpha} c_{\alpha}\left(\hat{b}_{\alpha}^{\dagger}+\hat{b}_{\alpha}\right)$, where $c_{\alpha}$ are the coupling parameters and $\hat{v}$ a coupling operator acting in the state space of the system.

Within the Floquet-Born-Markov approximation, the rates for the driven system are given by Fermi's golden rule [49-52],

$$
R_{j i}=\sum_{m=-\infty}^{\infty} R_{j i}^{(m)}, \quad R_{j i}^{(m)}=\frac{2 \pi}{\hbar}\left|v_{j i}(m)\right|^{2} g\left(\varepsilon_{j}-\varepsilon_{i}-m \hbar \omega\right) .
$$

Here $v_{j i}(m)=\frac{\omega}{2 \pi} \int_{0}^{2 \pi / \omega} d t e^{i m \omega t}\langle j(t)|\hat{v}| i(t)\rangle$ are the Fourier coefficients of the coupling matrix elements, where the index $m$ accounts for the absorption or emission of $|m|$ energy quanta $\hbar \omega$ due to the driving. The quantity

$$
g(E)=\frac{J(E)}{e^{\beta E}-1}=g(-E) e^{-\beta E}
$$

is the bath correlation function, determined by the inverse temperature $\beta=1 / T$ (the Boltzmann constant is set to one) and the spectral density,

$$
J(E)=\sum_{\alpha} c_{\alpha}^{2}\left[\delta\left(E-\hbar \omega_{\alpha}\right)-\delta\left(E+\hbar \omega_{\alpha}\right)\right]=-J(-E)
$$

We assume Ohmic baths characterized by a spectral density that increases linearly with $E, J(E) \propto E$.

In the autonomous system, Eq. (3) simplifies to

$$
R_{j i}=\sum_{b \in\{1,2\}} R_{j i}^{(b)}, \quad R_{j i}^{(b)}=\frac{2 \pi}{\hbar}\left|v_{j i}^{(b)}\right|^{2} g_{b}\left(E_{j}-E_{i}\right)
$$

Here $v_{j i}^{(b)}=\left\langle j\left|\hat{v}^{(b)}\right| i\right\rangle$ now denote the matrix elements of the coupling operator of heat bath $b$ with respect to the eigenstates $|i\rangle$ with energy $E_{i}$. The rate is further characterized by the correlation functions $g_{b}(E)=J_{b}(E)\left[\exp \left(\beta_{b} E\right)-1\right]^{-1}$ of both baths, with spectral density $J_{b}(E)$ and inverse temperature $\beta_{b}$.

Later we will see that the rate-asymmetry matrix

$$
A_{i j}=R_{i j}-R_{j i}
$$

plays a major role since many properties of the system depend on this matrix only. In the time-periodically driven case, it reads

$$
\begin{aligned}
A_{i j} & =\sum_{m=-\infty}^{\infty} A_{i j}^{(m)}, \\
A_{i j}^{(m)} & =R_{i j}^{(m)}-R_{j i}^{(m)}=\frac{2 \pi}{\hbar}\left|v_{j i}(m)\right|^{2} J\left(\varepsilon_{j}-\varepsilon_{i}-m \hbar \omega\right),
\end{aligned}
$$

whereas for the autonomous system one has

$$
\begin{aligned}
A_{i j} & =\sum_{b \in\{1,2\}} A_{i j}^{(b)}, \\
A_{i j}^{(b)} & =R_{i j}^{(b)}-R_{j i}^{(b)}=\frac{2 \pi}{\hbar}\left|v_{j i}^{(b)}\right|^{2} J_{b}\left(E_{j}-E_{i}\right) .
\end{aligned}
$$

Note that the rate-asymmetry matrix is independent of the bath temperature(s).

In contrast to equilibrium, a nonequilibrium steady state can retain a constant energy flow through the system. For the periodically driven system, the transition described by the rate $R_{j i}^{(m)}$ causes a change of the bath energy by $\varepsilon_{i}-\varepsilon_{j}+m \hbar \omega$.
The total energy flow from the system to the bath is thus given by

$$
Q(t)=\sum_{i j m}\left(\varepsilon_{i}-\varepsilon_{j}+m \hbar \omega\right) R_{j i}^{(m)} p_{i}(t) .
$$

Note that also pseudotransitions described by rates $R_{i i}^{(m \neq 0)}$ contribute to the heat flow [58]. These transitions change the state of the bath, but not that of the system. For the autonomous system the energy flow into bath $b$ reads

$$
Q_{b}(t)=\sum_{i j}\left(E_{i}-E_{j}\right) R_{j i}^{(b)} p_{i}(t) .
$$

\section{B. Master equation for the ideal quantum gas}

We now generalize the single-particle problem to a gas of $N$ indistinguishable, noninteracting particles. In our approach we assume the total particle number $N$ to be fixed, like in the canonical ensemble. For our considerations the canonical description poses the advantage that it contains the singleparticle case as the natural limit $N=1$, and does not require to define new terms describing the particle exchange with the bath.

The many-body Hilbert space is spanned by Fock states enumerated by the occupation numbers of the $M$ singleparticle states, $\boldsymbol{n}=\left(n_{1}, n_{2}, \ldots, n_{M}\right)$. To obtain the many-body rate equations, we replace the single-particle jump operators $\hat{L}_{i j}=|i\rangle\langle j|$ in Eq. (1) with their Fock-space representation

$$
\hat{L}_{i j}=\hat{a}_{i}^{\dagger} \hat{a}_{j} \text {. }
$$

Here $\hat{a}_{i}$ denotes the annihilation operator of a particle, boson or fermion, in the single-particle mode $i$. Quantum jumps still correspond to processes transferring a single particle from one mode to another. The validity of the full rotating-wave approximation is, thus, still determined by the single-particle problem. Moreover, the total particle number $N$ is conserved by the dynamics.

As before, the dynamics of the many-body occupation probabilities $p_{\boldsymbol{n}}=\langle\boldsymbol{n}|\hat{\rho}| \boldsymbol{n}\rangle$ decouple from the off-diagonal elements, which decay over time. The corresponding equations of motion are now given by (see Appendix A for details)

$$
\dot{p}_{\boldsymbol{n}}(t)=\sum_{i j}\left(1+\sigma n_{j}\right) n_{i}\left[R_{i j} p_{\boldsymbol{n}_{j i}}(t)-R_{j i} p_{\boldsymbol{n}}(t)\right]
$$

which is the many-body generalization of the Pauli master equation (2). Here $\boldsymbol{n}_{j i}=\left(n_{1}, \ldots, n_{i}-1, \ldots, n_{j}+1, \ldots\right)$ denotes the occupation numbers obtained from $\boldsymbol{n}$ by transferring one particle from $i$ to $j$. The effective transition rate depends on the quantum statistics via the choice of $\sigma$, with $\sigma=1$ for bosons (reflecting the enhancement of transitions into occupied states) and $\sigma=-1$ for fermions (reflecting the Pauli exclusion principle). The classical case of distinguishable (Boltzmann) particles corresponds to $\sigma=0$; here the transition rates are independent of the occupation of the final state. ${ }^{1}$

\footnotetext{
${ }^{1}$ The bosonic master equation (13) with $\sigma=1$, as well as the corresponding mean-field equation (31), also resemble rate equations that are used to describe stochastic processes in classical systems, as we mention them already in the introduction.
} 
For the periodically driven ideal gas the energy flow from the system into the bath is given by

$$
\begin{aligned}
Q(t) & =\sum_{m} \sum_{n} \sum_{i j}\left(\varepsilon_{i}-\varepsilon_{j}+m \hbar \omega\right) R_{j i}^{(m)}\left(1+\sigma n_{j}\right) n_{i} p_{\boldsymbol{n}}(t) \\
& =\sum_{m} \sum_{i j}\left(\varepsilon_{i}-\varepsilon_{j}+m \hbar \omega\right) R_{j i}^{(m)}\left[\left\langle\hat{n}_{i}\right\rangle(t)+\sigma\left\langle\hat{n}_{i} \hat{n}_{j}\right\rangle(t)\right] .
\end{aligned}
$$

Analogously, for the autonomous ideal gas the energy flow into bath $b$ reads

$$
\begin{aligned}
Q_{b}(t) & =\sum_{n} \sum_{i j}\left(E_{i}-E_{j}\right) R_{j i}^{(b)}\left(1+\sigma n_{j}\right) n_{i} p_{\boldsymbol{n}}(t) \\
& =\sum_{i j}\left(E_{i}-E_{j}\right) R_{j i}^{(b)}\left[\left\langle\hat{n}_{i}\right\rangle(t)+\sigma\left\langle\hat{n}_{i} \hat{n}_{j}\right\rangle(t)\right] .
\end{aligned}
$$

\section{Nonequilibrium steady state}

In the following we are interested in the properties of the steady state of the ideal quantum gas, whose density operator is simply denoted by $\hat{\rho} .^{2}$ It is diagonal in the occupation number basis,

$$
\hat{\rho}=\sum_{n} p_{n}|\boldsymbol{n}\rangle\langle\boldsymbol{n}|,
$$

with $p_{n}$ determined by solving Eq. (13) for $\dot{p}_{\boldsymbol{n}}=0$. The uniqueness of the steady state [57] is inherited from the single-particle system, since every Fock state is connected to every other Fock state by a sequence of allowed single-particle transitions when this is assumed for the single-particle system.

The steady-state expectation value of an arbitrary observable $\hat{o}$ is denoted by

$$
\langle\hat{o}\rangle=\operatorname{tr}(\hat{\rho} \hat{o}) .
$$

Expectation values that we consider in the following are the mean occupations that we denote by

$$
\bar{n}_{i}=\left\langle\hat{n}_{i}\right\rangle \text {, }
$$

with the number operator $\hat{n}_{i}=\hat{a}_{i}^{\dagger} \hat{a}_{i}$ and the two-particle correlations $\left\langle\hat{n}_{i} \hat{n}_{j}\right\rangle$ or, rather, their nontrivial part,

$$
\zeta_{i j}=\left\langle\hat{n}_{i} \hat{n}_{j}\right\rangle-\bar{n}_{i} \bar{n}_{j}=\left\langle\left(\hat{n}_{i}-\bar{n}_{i}\right)\left(\hat{n}_{j}-\bar{n}_{j}\right)\right\rangle .
$$

For the scenarios depicted in Fig. 1 the steady state of the system will be a nonequilibrium steady state. This can be illustrated already on the level of the single-particle problem (2). Let us first recapitulate the case of thermal equilibrium. The transitions induced by a single bath of inverse temperature $\beta$ in an autonomous system are described by rates that obey

$$
\frac{R_{j i}}{R_{i j}}=e^{-\beta\left(E_{j}-E_{i}\right)} .
$$

\footnotetext{
${ }^{2}$ Whenever we are discussing transient behavior and time-dependent quantities (which happens only a few times) this is indicated by writing out explicitly the time argument. For example, $\hat{\rho}(t)$ denotes the time-dependent density operator or $\langle\hat{o}\rangle(t)$ a time-dependent expectation value. Otherwise, i.e., when writing $\hat{\rho}$ or $\langle\hat{o}\rangle$, we are always referring to steady-state quantities.
}

This can be inferred from Eq. (6) for the case of a single bath. This condition implies that the steady state, obtained by solving Eq. (2) is given by the Gibbs state with $p_{i}=Z^{-1} e^{-\beta E_{i}}$ and $Z=\sum_{i} e^{-\beta E_{i}}$. For this equilibrium state, the sum on the right-hand side of Eq. (2) vanishes term by term. Thus, the net probability flux between two states $i$ and $j$ vanishes. This is the property of detailed balance, which is characteristic for the thermodynamic equilibrium.

The rates characterizing the periodically driven system, Eq. (3), or the autonomous system coupled to two heat baths of different temperature, Eq. (6), are a sum of rates corresponding to different energy changes in the bath or to different bath temperatures, respectively. As a consequence, they do not obey condition (20) anymore. This implies that, generally, the steady state also does not fulfill detailed balance anymore. While the net probability flux into a state $i$, determined by the right-hand side of Eq. (2), still has to vanish, the probability current from a certain state $j$ to state $i$ can be nonzero; i.e., the sum in Eq. (2) does not vanish term by term. The lack of detailed balance characterizes a nonequilibrium steady state. In contrast to the equilibrium state, which is determined by the temperature of the bath only, the nonequilibrium steady state depends on the very details of the bath(s) (the temperature, the coupling operator, and the spectral density). This makes the computation of the many-body nonequilibrium steady state a difficult problem. However, it also offers opportunities to realize states with properties that are hard (or impossible) to achieve in equilibrium.

\section{Model systems}

Throughout this paper, we illustrate our findings using three different model systems. Let us briefly define them here. Note that our results are not limited to these example systems.

The first model system is a tight-binding chain of $M$ lattice sites. It is described by the Hamiltonian

$$
\hat{H}=-J \sum_{\ell=1}^{M-1}\left(\hat{c}_{\ell}^{\dagger} \hat{c}_{\ell+1}+\text { H.c. }\right)
$$

wherein $\hat{c}_{\ell}\left(\hat{c}_{\ell}^{\dagger}\right)$ denotes the annihilation (creation) operator for a particle at site $\ell$. The single-particle eigenstates $|i\rangle$, with $i=0,1, \ldots, M-1$, are delocalized. They are described by wave functions $\langle\ell \mid i\rangle \propto \sin \left(k_{i} \ell\right)$, with wave numbers $k_{i}=(i+$ $1) \pi /(M+1)$ and possess energies $E_{i}=-2 J \cos \left(k_{i}\right)$ between $-2 J$ and $2 J$. As sketched in Fig. 2(a), the chain is coupled to two baths, on the left and right end of the chain. The left (right) bath is locally coupled to the first (next-to-last) site of the chain via the coupling operators $\hat{v}_{1}=\gamma_{1} \hat{c}_{1}^{\dagger} \hat{c}_{1}$ and $\hat{v}_{2}=\gamma_{2} \hat{c}_{M-1}^{\dagger} \hat{c}_{M-1}$, respectively. ${ }^{3}$ This coupling describes a bath-induced fluctuation of the on-site energy. The steady state will depend on the coupling strength only through their relative weight $\gamma_{2} / \gamma_{1}$, while their absolute weight determines how fast the system relaxes. The temperatures of the baths are different from each other. We will, moreover, mainly focus on the interesting case

\footnotetext{
${ }^{3}$ We avoid the choice of coupling the second bath to the last site $M$ since, for such a symmetric configuration, the generic effect of fragmented Bose condensation [23] is absent.
} 
(a)

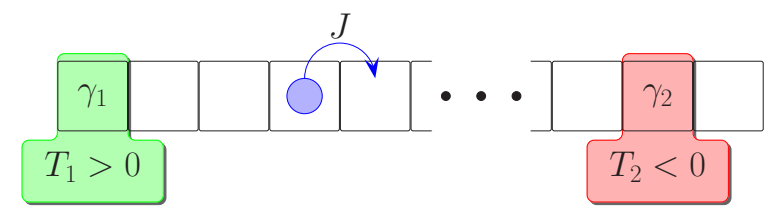

(b)

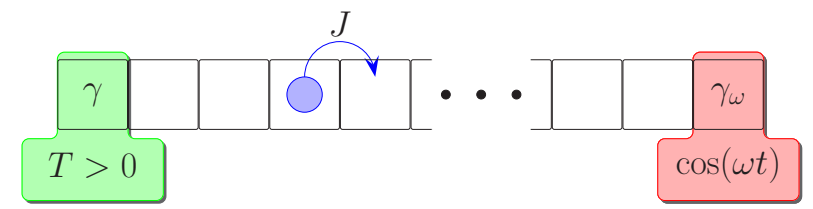

FIG. 2. (Color online) Two model systems. (a) Tight-binding chain coupled to two heat baths of respective temperatures $T_{1}$ and $T_{2}$ and coupling strengths $\gamma_{1}$ and $\gamma_{2}$. (b) Tight-binding chain subjected to a time-periodic potential modulation at one end with driving strength $\gamma_{\omega}$ and angular frequency $\omega$ and coupled to a heat bath of temperature $T$ at the other end with coupling strength $\gamma$.

where one of the baths is population inverted. For such a situation the notion of the single-particle ground state becomes meaningless, allowing for fragmented Bose condensation with multiple condensates [23], see Sec. IV below. We model the population-inverted bath by a negative temperature $T_{2}<0$ and a spectrum that is bounded from above $\left(\omega_{\alpha}<0\right)$.

The second model system is also given by a tight-binding chain of $M$ sites. However, instead of coupling it to a second bath, the chain is periodically driven in time. Its Hamiltonian is given by

$$
H(t)=-J \sum_{\ell=1}^{M-1}\left(\hat{c}_{\ell}^{\dagger} \hat{c}_{\ell+1}+\text { H.c. }\right)+\gamma_{\omega} J \cos (\omega t) \hat{c}_{M}^{\dagger} \hat{c}_{M},
$$

with the dimensionless driving strength $\gamma_{\omega}$ and angular frequency $\omega$. The coupling to a bath of inverse temperature $\beta$ is realized via the coupling operator $\hat{v}=\gamma \hat{c}_{1}^{\dagger} \hat{c}_{1}$, as depicted in Fig. 2(b). The steady state will depend on the dimensionless driving strength $\gamma_{\omega}$, which determines the single-particle Floquet modes and the structure of the rate matrix $R_{i j}$. However, the coupling strength to the heat bath $\gamma$ has no impact on the steady state, but rather determines how fast the system relaxes.

Finally, as a third model, we consider a system of $M$ single-particle states with the transition rates $R_{i j}$ given by uncorrelated random numbers, independently drawn from an exponential distribution,

$$
P\left(R_{j i}\right)=\lambda^{-1} \exp \left(-\lambda R_{j i}\right) .
$$

The parameter $\lambda$ controls the time scale of the relaxation, but does not influence the steady state. The diagonal elements $R_{i i}$ can be set to 0 as they drop out of all relevant equations [such as Eq. (2)]. This choice of rates clearly models a nonequilibrium situation, since detailed balance is violated almost surely. It is motivated by the rates computed for fully chaotic periodically driven quantum systems coupled to a heat bath [59]. A concrete example is given by the kicked rotor coupled to a bath, which is discussed for single particles in Ref. [60] and for many particles in the supplemental material of Ref. [23].

\section{METHODS}

In this paper we are interested in the properties of nonequilibrium steady states (16) of driven dissipative ideal quantum gases of $N$ particles, described by the master equation (2) with jump operators (12) or, equivalently, by the rate equation (13). Even though the particles are noninteracting, finding the steady state is a true many-body problem. Unlike in equilibrium, the many-particle solution cannot be obtained from the single-particle solution in a straightforward manner. This is a consequence of the interaction with the bath and reflected in the fact that the right-hand side of the master equation (2) is quadratic in the jump operators (12) and, thus, quartic in the bosonic or fermionic field operators $\hat{a}_{i}^{(\dagger)}$. As a consequence, Eq. (13) quickly becomes intractable when the particle number is increased. Therefore, it is crucial to develop and apply suitable methods for the approximate treatment of the problem. This is done in this section.

In the following, we first describe quantum-jump-type Monte Carlo simulations based on averaging over random walks in the classical space of sharp occupation numbers. This numerical method is quasiexact (the statistical error is controlled) and allows for the treatment of moderately large systems. In order to treat even larger systems and to obtain an intuitive picture of the dynamics, we then describe a mean-field theory, which is based on a description in terms of the mean occupations $\bar{n}_{i}$. Finally, we augment the mean-field theory by taking into account fluctuations given by nontrivial two-particle correlations.

\section{A. Monte Carlo simulations}

Quantum-jump Monte Carlo simulations [61,62] are an efficient method for computing the time evolution of open quantum systems described by a Markovian master equation of Lindblad form. Instead of integrating the time evolution of the full density matrix, the method is based on integrating the time evolution of single states (the Monte Carlo wave function). In doing so, the dissipative effect of the environment is included by interrupting the continuous time evolution by a sudden quantum jump, described by one of the jump operators. When such a quantum jump occurs, and which one, is drawn from a suitable probability distribution. The time evolution of expectation values can then be obtained by averaging over an ensemble of Monte Carlo wave functions. The error depends on the ensemble size and can, in principle, be made arbitrarily small.

When treating the master equation (2) with jump operators (12) we encounter a convenient situation. The dissipation can be described by jump operators (12) that transfer a particle from one single-particle eigenstate (or Floquet state) to another one, i.e., between two states of sharp occupation numbers $\boldsymbol{n}$. At the same time, these occupation numbers are conserved by the evolution generated by the system Hamiltonian, since we are dealing with a system of noninteracting particles. Therefore, the time evolution is exhausted by taking into account quantum jumps. This corresponds to a random walk in the classical space spanned by the Fock states $|\boldsymbol{n}\rangle$ (not their superpositions). The Monte Carlo wave function $|\boldsymbol{n}(t)\rangle$ jumps between Fock states $\left|\boldsymbol{n}_{k}\right\rangle$, in which it resides for time intervals of 
length $t_{k}$,

$$
|\boldsymbol{n}(t)\rangle=\left|\boldsymbol{n}_{k}\right\rangle \quad \text { with } k \text { such that } T_{k-1} \leqslant t<T_{k},
$$

where $T_{k}=\sum_{l=1}^{k} t_{l}$.

We use the Gillespie algorithm [63] in order to compute the time evolution. At the beginning, the system is prepared according to the chosen initial conditions. Then the algorithm alternates between the following two steps. (i) The time interval $t_{k}$ determining how long the system will remain in the current state is drawn randomly from an exponential distribution $P\left(t_{k}\right) \propto \exp \left[-t_{k} / \bar{t}\left(\boldsymbol{n}_{k}\right)\right]$ with mean dwell time,

$$
\bar{t}\left(\boldsymbol{n}_{k}\right)=\frac{1}{\sum_{i, j} R_{i j}\left(1+\sigma n_{i}\right) n_{j}} .
$$

(ii) The new state with occupation $\boldsymbol{n}_{k+1}$ is drawn randomly with branching probability reflecting the many-body transition rates $R_{j i}\left(1+\sigma n_{j}\right) n_{i}$. Since only single-particle jumps are involved in Eq. (13), the next state is obtained from the current state by transferring a particle from a randomly drawn departure state $i$ to the randomly drawn target state $j$. This single-particle jump has the probability

$$
P\left(i \rightarrow j, \boldsymbol{n}_{k}\right)=\bar{t}\left(\boldsymbol{n}_{k}\right) R_{j i}\left(1+\sigma n_{j}\right) n_{i} .
$$

These two steps are repeated until $T_{k}=\sum_{l=1}^{k} t_{l}$ exceeds the desired evolution time $t_{\text {fin }}$.

From an ensemble of $L$ Monte Carlo wave functions $\left|\boldsymbol{n}^{(\alpha)}(t)\right\rangle$ labeled by $\alpha=1,2, \ldots, L$, one can then compute the expectation value of an observable $\hat{o}$,

$$
\langle\hat{o}\rangle_{\text {ensemble }}(t)=\frac{1}{L} \sum_{\alpha=1}^{L}\left\langle\boldsymbol{n}^{(\alpha)}(t)|\hat{o}| \boldsymbol{n}^{(\alpha)}(t)\right\rangle .
$$

Figure 3 shows the time evolution of the mean occupations $\left\langle\hat{n}_{i}\right\rangle(t)$ for $N=100$ particles on $M=5$ states for a single Monte Carlo wave function (thin lines) and for an ensemble

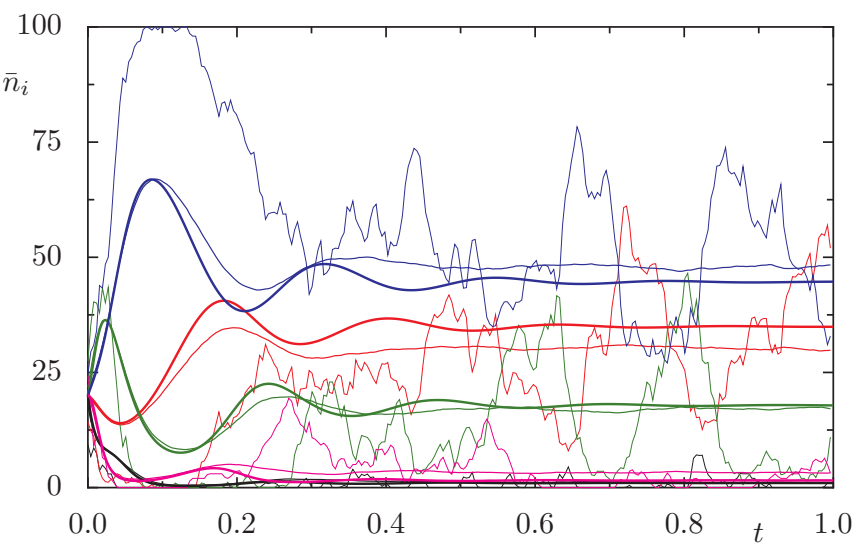

FIG. 3. (Color online) Time evolution of the mean occupations $\bar{n}_{i}(t)$ for one realization of the random-rate model for $M=5$ states and $N=100$ particles. Time is measured in units of the inverse mean rate $\lambda$ [see Eq. (23)]. Initially, each single-particle state is occupied with the same probability. The thin lines are obtained from a single Monte Carlo wave function, the intermediate lines from an ensemble of $L=1000$ Monte Carlo wave functions, and the thick lines from mean-field theory. The mean-field results show small systematic deviations from the Monte Carlo result. with $L=1000$ (intermediate lines). One can clearly observe the relaxation to a steady state reached after a relaxation time of $\tau_{r} \approx 0.5$. Slight temporal fluctuations observed for times $t>\tau_{r}$ decrease with ensemble size $L$. The mean-field theory (thick lines) described below predicts the occupations rather well, but with small systematic deviations from the Monte Carlo result.

When computing steady-state expectation values $\langle\hat{o}\rangle$, the effect of temporal fluctuations can be reduced by combining ensemble averaging with time averaging,

$$
\langle\hat{o}\rangle=\frac{1}{L} \sum_{\alpha=1}^{L} \sum_{k} \frac{\left\langle\boldsymbol{n}_{k}^{(\alpha)}|\hat{o}| \boldsymbol{n}_{k}^{(\alpha)}\right\rangle}{t_{k}} .
$$

Here it is useful to constrain the inner sum to $k>k_{r}^{(\alpha)}$, with $k_{r}^{(\alpha)}$ such that $T_{k_{r}^{(\alpha)}}>t_{r}$, in order to exclude the transient relaxation process from the time average. Since we assume that every state is connected with all the other states by a sequence of transitions with nonvanishing rates, one can obtain accurate steady-state expectation values from a single Monte Carlo trajectory, provided $t_{\text {fin }}$ is sufficiently large so that the system forgets its initial state after a certain correlation time. Averaging over a long time is, therefore, equivalent to averaging over an ensemble. We determine these uncertainties according to the Gelman-Rubin criterion [64], generally setting the relative uncertainties below $1 \%$ (small enough to make statistical fluctuations barely noticeable in any figure). For a bosonic system, this allows us to access particle numbers $N \sim 10^{5}$ for $M=100$ single-particle states.

\section{B. Mean-field theory}

In order to treat even larger systems and to gain some intuitive understanding of the nonequilibrium steady state of ideal quantum gases, it is desirable to use also analytical methods. One of them is a mean-field description of the system in terms of the mean occupations $\bar{n}_{i}$ [23].

The time evolution of the mean occupations is given by the equations

$$
\begin{aligned}
\frac{d}{d t} \bar{n}_{i}(t)= & \operatorname{tr}\left[\hat{n}_{i} \frac{d}{d t} \hat{\rho}(t)\right]=\sum_{j} R_{i j}\left\{\left[\bar{n}_{j}(t)+\sigma\left\langle\hat{n}_{i} \hat{n}_{j}\right\rangle(t)\right]\right. \\
& \left.-R_{j i}\left[\bar{n}_{i}(t)+\sigma\left\langle\hat{n}_{i} \hat{n}_{j}\right\rangle(t)\right]\right\}
\end{aligned}
$$

for all $i$ (see Appendix B). Here we encounter the typical hierarchy: The time evolution of single-particle correlations (expectation values of operators that are quadratic in the field operators) is governed by two-particle correlations (expectation values of operators that are quartic in the field operators). The evolution of the latter will, in turn, be determined by three-particle correlations, and so on.

In order to obtain a closed set of equations in terms of the mean occupations, we employ the factorization approximation

$$
\left\langle\hat{n}_{i} \hat{n}_{j}\right\rangle(t)=\bar{n}_{i}(t) \bar{n}_{j}(t)+\zeta_{i j}(t) \approx \bar{n}_{i}(t) \bar{n}_{j}(t)
$$

for $i \neq j$. Here nontrivial correlations are neglected, $\zeta_{i j}(t) \approx$ 0 , so that two-particle correlations are approximated by a product of single-particle expectation values as if Wick's theorem was valid. In this way we arrive at the set of nonlinear 
mean-field equations

$$
\frac{d}{d t} \bar{n}_{i}(t) \approx \sum_{j}\left\{R_{i j} \bar{n}_{j}(t)\left[1+\sigma \bar{n}_{i}(t)\right]-R_{j i} \bar{n}_{i}(t)\left[1+\sigma \bar{n}_{j}(t)\right]\right\} .
$$

In the classical case of distinguishable particles, which can be shown to be captured by $\sigma=0$, the mean-field equation is exact. In this case, the equations of motion for the mean occupations $\bar{n}_{i}(t)$ are of the same form as the single-particle master equation (2) for the probabilities $p_{i}(t)$. Therefore, in the classical system the mean occupations are determined by the single-particle problem and read $\bar{n}_{i}(t)=p_{i}(t) N$. In contrast, for quantum gases of indistinguishable bosons or fermions the dynamics and the steady state will depend in a nontrivial way on the total particle number. In this case, the classical solution can still be an approximate solution of the quantum system as long as $\bar{n}_{i} \ll 1$ for all $i$, so that two-particle correlations $\left\langle\hat{n}_{i} \hat{n}_{j}\right\rangle$ are negligible. However, as soon as the quantum degenerate regime is reached, where $\bar{n}_{i} \gtrsim 1$ at least for some $i$, quantum statistics and with that the particle number will matter.

The mean-field equations of motion can also be obtained by making a Gaussian ansatz,

$$
\hat{\rho}_{g}=\frac{1}{Z} \exp \left[-\sum_{i} \eta_{i} \hat{n}_{i}\right]
$$

with partition function $Z$ for the many-body density operator. For this ansatz the mean occupations are given by

$$
\left\langle\hat{n}_{i}\right\rangle_{g}=\frac{1}{e^{\eta_{i}}-\sigma} .
$$

Thus, the $M$ parameters defining the Gaussian state are determined completely by the $M$ mean occupations, $\eta_{i}=$ $\ln \left(\left\langle\hat{n}_{i}\right\rangle_{g}^{-1}+\sigma\right)$, as they can be obtained by solving the mean-field equations (31). Nontrivial correlations vanish and multiparticle correlation functions can be decomposed into products of single-particle correlations determined by Wick decomposition. For the two-particle correlations the Gaussian ansatz gives [65]

$$
\left\langle\hat{n}_{i} \hat{n}_{j}\right\rangle_{g}= \begin{cases}\left\langle\hat{n}_{i}\right\rangle_{g}\left[(1+\sigma)\left\langle\hat{n}_{i}\right\rangle_{g}+1\right] & \text { for } i=j, \\ \left\langle\hat{n}_{i}\right\rangle_{g}\left\langle\hat{n}_{j}\right\rangle_{g} & \text { for } i \neq j,\end{cases}
$$

for bosons $(\sigma=1)$ and fermions $(\sigma=-1)$. For $i \neq j$ we find $\left\langle\hat{n}_{i} \hat{n}_{j}\right\rangle_{g}=\left\langle\hat{n}_{i}\right\rangle_{g}\left\langle\hat{n}_{j}\right\rangle_{g}$. Therefore, starting from Eq. (29) and making the Gaussian ansatz for the density operator, we recover the mean-field equations of motion (31) with $\bar{n}_{i}(t)=\left\langle\hat{n}_{i}\right\rangle_{g}$.

With the quantities $\left\langle\hat{n}_{i}^{2}\right\rangle_{g}$, the Gaussian ansatz also determines the fluctuations of the occupations $\hat{n}_{i}$ as well as of the total particle number $\hat{N}=\sum_{i} \hat{n}_{i}$. One finds

$$
\left\langle\left(\hat{n}_{i}-\left\langle\hat{n}_{i}\right\rangle_{g}\right)^{2}\right\rangle_{g}=\left\langle\hat{n}_{i}^{2}\right\rangle_{g}-\left\langle\hat{n}_{i}\right\rangle_{g}^{2}=\left\langle\hat{n}_{i}\right\rangle_{g}+\sigma\left\langle\hat{n}_{i}\right\rangle_{g}^{2}
$$

and

$$
\begin{aligned}
\left\langle\left(\hat{N}-\langle\hat{N}\rangle_{g}\right)^{2}\right\rangle_{g}= & \sum_{i}\left(\left\langle\hat{n}_{i}^{2}\right\rangle_{g}-\left\langle\hat{n}_{i}\right\rangle_{g}^{2}\right) \\
& +\sum_{i, j \neq i}\left(\left\langle\hat{n}_{i} \hat{n}_{j}\right\rangle_{g}-\left\langle\hat{n}_{i}\right\rangle_{g}\left\langle\hat{n}_{j}\right\rangle_{g}\right) \\
= & \sum_{i}\left\langle\left(\hat{n}_{i}-\left\langle\hat{n}_{i}\right\rangle_{g}\right)^{2}\right\rangle_{g} .
\end{aligned}
$$

The Gaussian state does not describe a system with a sharp particle number, so we can only require that the mean particle number obeys

$$
\langle\hat{N}\rangle_{g}=N
$$

Fluctuations of the total particle number are an immediate consequence of enforcing trivial correlations $\left\langle\hat{n}_{i} \hat{n}_{j}\right\rangle=\bar{n}_{i} \bar{n}_{j}$ for $i \neq j$ (unless also the occupations of the individual states $i$ are sharp so that their number fluctuations $\left\langle\hat{n}_{i}^{2}\right\rangle-\bar{n}_{i}^{2}$ vanish). This can be seen from Eq. (36), where we have not yet used the properties of the Gaussian state like in Eq. (37). It is intuitively clear that a sharp total particle number induces nontrivial correlations among the occupations. If the measurement of the occupation $\hat{n}_{i}$ gives a value $n_{i}$ that is smaller (larger) than the expectation value $\bar{n}_{i}$, a sharp total particle number implies that the number of particles in all other states is given by $N-n_{i}$ and, thus, larger (smaller) than the original expectation value $N-\bar{n}_{i}$. As a consequence, the probability of measuring a certain value $n_{j}$ of the occupation $\hat{n}_{j}$ with $j \neq i$ will depend on the value $n_{i}$ measured for the occupation $\hat{n}_{i}$.

The role played by fluctuations of the total particle number becomes less and less important in large systems. Namely, the variance of the total particle number (37) is the sum over the variances of the occupations of individual modes (35), which are intensive. Thus, the fluctuations of the total particle number grow in a subextensive fashion like the square root of the system size. That is, the relative fluctuations of the total particle number vanish in the limit of large systems. This is the mechanism underlying the equivalence of the canonical and the grand-canonical ensembles. There is one important exception, however. This is the case of Bose-Einstein condensation, where in a bosonic system a mode $i$ acquires a macroscopic occupation. If the total particle number is not conserved, also the number fluctuations of the condensate mode will be as large as the number of condensed particles; in this case the right-hand side of Eq. (35) is dominated by the second term. The extensive number fluctuations in the condensate mode will then dominate the sum of Eq. (37) and give rise to extensive total number fluctuations, which are non-negligible in large systems. This phenomenon is know as the grand-canonical fluctuation catastrophe [66].

However, one should note that the dynamics of the mean occupations $\bar{n}_{i}(t)$ described by Eq. (29) do not depend on the occupation number fluctuations of the modes (the term $j=i$ vanishes so that $\left\langle\hat{n}_{i}^{2}\right\rangle$ does not enter on the right-hand side). The mean-field equations of motion (31) can, therefore, provide a good approximation to the mean occupations $\bar{n}_{i}$ also in systems featuring Bose condensation (see Ref. [23]). This can be seen also in Fig. 3, where, despite the fact that half of the particles occupy a single mode, mean-field theory accurately describes both the transient and the long-time behavior of the mean occupations.

The grand-canonical ensemble of an ideal quantum gas in equilibrium with inverse temperature $\beta$ and chemical potential $\mu$ is described by a Gaussian density operator (32) with $\eta_{i}=\beta\left(E_{i}-\mu\right)$. The mean occupations Eq. 33 follow the Bose-Einstein (Fermi-Dirac) distribution for $\sigma=1(\sigma=-1)$. The grand-canonical ideal gas is thus described exactly within the mean-field theory. This can be seen explicitly by plugging the Gaussian state $p_{\boldsymbol{n}} \propto \prod_{i} e^{-\beta\left(E_{i}-\mu\right) n_{i}}$ (solving the mean-field 
equation) into the full many-body rate equations (13). By employing condition (20), which is fulfilled in an equilibrium situation, one can see that the sum on the right-hand side vanishes term by term. This implies also that the equilibrium state obeys detailed balance as it should. Deviations from mean-field theory occur as a consequence of two factors: (i) the assumption of a sharp total particle number and (ii) the violation of the detailed-balance condition (20).

Both factors (i) and (ii) are independent of each other, as can be illustrated using two examples. The canonical equilibrium state with sharp particle number is characterized by the nonGaussian probabilities

$$
p_{\boldsymbol{n}}= \begin{cases}\frac{1}{Z_{N}} \exp \left(-\sum_{i} \beta E_{i} n_{i}\right) & \text { if } \sum_{i} n_{i}=N, \\ 0 & \text { otherwise }\end{cases}
$$

with the partition function $Z_{N}$. This state can be obtained by projecting the Gaussian state onto the subspace of sharp total particle number $N$. As a consequence of the sharp particle number, it does not solve the mean-field equation, as discussed above. However, it still obeys detailed balance. Namely, plugging it into Eq. (13) the sum on the right-hand side vanishes term by term as long as the condition (20) is fulfilled. On the other hand, we can allow the particle number to fluctuate freely, but violate condition (20). Then it will generally not be possible to find a solution of the mean-field form (32) that solves the many-body rate equations (13), because the number of independent equations exceeds the number of parameters $\eta_{i}$. In the following, we are interested in the situation where a system of sharp particle number is driven into a steady state far away from equilibrium, so that both factors (i) and (ii) are present. Here the mean-field theory can still provide a good approximation, as can be checked by comparing it to quasiexact results obtained from Monte Carlo simulations.

Within the mean-field approximation, the heat flow for the autonomous system to bath $b$, given by Eq. (15), takes the form

$$
Q^{(b)}(t)=\sum_{i, j \neq i}\left(E_{i}-E_{j}\right) R_{j i}^{(b)} \bar{n}_{i}(t)\left[1+\sigma \bar{n}_{j}(t)\right] .
$$

The heat flow from the periodically driven ideal gas into the heat bath (14) reads

$$
\begin{aligned}
Q(t)= & \sum_{m} \sum_{i, j \neq i}\left(\varepsilon_{i}-\varepsilon_{j}+m \hbar \omega\right) R_{j i}^{(m)} \bar{n}_{i}(t)\left[1+\sigma \bar{n}_{j}(t)\right] \\
& +\sum_{m} \sum_{i} m \hbar \omega R_{i i}^{(m)}\left[\bar{n}_{i}(t)+\sigma\left\langle\hat{n}_{i}^{2}\right\rangle(t)\right] .
\end{aligned}
$$

Here the second sum captures the heat flow related to pseudotransitions [see discussion below Eq. (10)]. Their contribution depends on $\left\langle\hat{n}_{i}^{2}\right\rangle$ and, thus, on the occupation number fluctuations of the modes. However, as discussed above, in a bosonic system of sharp total particle number and where some modes feature macroscopic occupation, the Gaussian expectation value $\left\langle\hat{n}_{i}^{2}\right\rangle_{g}=\left\langle\hat{n}_{i}\right\rangle_{g}\left[2\left\langle\hat{n}_{i}\right\rangle_{g}+1\right]$ does generally not provide a good approximation for the condensate mode(s). Therefore, it might be useful to introduce another approximation for $\left\langle\hat{n}_{i}^{2}\right\rangle$ in an ad hoc fashion. Another possibility is to augment the mean-field theory such that it is able to treat systems with sharp particle number and, thus, with nontrivial two-particle correlations. Such a method is presented in the following section.

\section{Augmented mean-field theory}

By construction, the mean-field theory fails to take into account nontrivial two-particle correlations $\zeta_{i j}$ as they result from having a sharp total particle number and from driving the system out of equilibrium, so that the detailed-balance condition (20) is violated. The effects of a fluctuating total number of particles can be assessed by projecting the Gaussian state onto the subspace of $N$-particle states, $\hat{\rho}_{\text {proj }} \propto \hat{P}_{N} \hat{\rho}_{g} \hat{P}_{N}$ with $\hat{P}_{N}=\sum_{\boldsymbol{n} \mid \sum_{i} \hat{n}_{i}=N}|\boldsymbol{n}\rangle\langle\boldsymbol{n}|$. This introduces nontrivial correlations, which can be obtained from $\left\langle\hat{n}_{i} \hat{n}_{j}\right\rangle=\operatorname{tr}\left(\rho_{\text {proj }} \hat{n}_{i} \hat{n}_{j}\right)$. However, evaluating this matrix element is an onerous task even within efficient algorithms (see Appendix $\mathrm{C}$ for an example), since all $N$-particle Fock states have to be accounted for. Moreover, such an approach still does not include effects related to the breaking of detailed balance.

In order to include the effects of nontrivial occupation correlations and fluctuations by analytic means, we introduce an augmented mean-field theory. This approach includes the two-point correlation functions $\left\langle\hat{n}_{k} \hat{n}_{i}\right\rangle$ into the hierarchy of equations of motions. In the original full hierarchy, the corresponding equations of motion take the form

$$
\begin{aligned}
\frac{d}{d t}\left\langle\hat{n}_{k} \hat{n}_{i}\right\rangle= & \sum_{j}\left\{\sigma\left(A_{k j}+A_{i j}\right)\left\langle\hat{n}_{k} \hat{n}_{i} \hat{n}_{j}\right\rangle+R_{k j}\left\langle\hat{n}_{i} \hat{n}_{j}\right\rangle\right. \\
& +R_{i j}\left\langle\hat{n}_{k} \hat{n}_{j}\right\rangle-\left(R_{j k}+R_{j i}\right)\left\langle\hat{n}_{k} \hat{n}_{i}\right\rangle \\
& +\delta_{i k}\left[R_{k j}\left(\bar{n}_{j}+\sigma\left\langle\hat{n}_{k} \hat{n}_{j}\right\rangle\right)\right. \\
& \left.\left.+R_{j k}\left(\bar{n}_{k}+\sigma\left\langle\hat{n}_{k} \hat{n}_{j}\right\rangle\right)\right]\right\} \\
& -R_{i k}\left(\bar{n}_{k}+\sigma\left\langle\hat{n}_{k} \hat{n}_{i}\right\rangle\right)-R_{k i}\left(\bar{n}_{i}+\sigma\left\langle\hat{n}_{k} \hat{n}_{i}\right\rangle\right)
\end{aligned}
$$

Here, as well as in the rest of this section, we suppress time arguments. This equation still involves the third-order correlations $\left\langle\hat{n}_{k} \hat{n}_{i} \hat{n}_{j}\right\rangle$.

The hierarchy can be closed by assuming trivial threeparticle correlations. For that purpose we separate the number operators like $\hat{n}_{i}=\bar{n}_{i}+\hat{\zeta}_{i}$ into their mean values $\bar{n}_{i}$ and their fluctuations

$$
\hat{\zeta}_{i}=\hat{n}_{i}-\bar{n}_{i} \quad \text { with } \quad\left\langle\hat{\zeta}_{i}\right\rangle=0
$$

We now approximate

$$
\left\langle\hat{\zeta}_{k} \hat{\zeta}_{i} \hat{\zeta}_{j}\right\rangle=0
$$

while allowing, in contrast to mean-field theory, for nontrivial two-particle correlations $\zeta_{k i}=\left\langle\hat{\zeta}_{k} \hat{\zeta}_{i}\right\rangle$ [Eq. (19)]. Thus, the equations of motion for the mean occupations are given by

$$
\begin{aligned}
\frac{d \bar{n}_{k}}{d t}= & \sigma \sum_{j} A_{k j}\left[\bar{n}_{k} \bar{n}_{j}+\zeta_{k j}\right] \\
& +\sum_{j}\left(R_{k j} \bar{n}_{j}-R_{j k} \bar{n}_{k}\right),
\end{aligned}
$$

which is equivalent to the exact equation (29). The equations of motion for the nontrivial two-particle correlations are obtained from Eq. (42) by employing the approximation (44). It is 
nonlinear and reads (see Appendix D for details)

$$
\begin{aligned}
\frac{d \zeta_{k i}}{d t} \approx & \sum_{j}\left\{\sigma\left[A_{k j} \bar{n}_{k} \zeta_{i j}+A_{i j} \bar{n}_{i} \zeta_{k j}+\left(A_{k j}+A_{i j}\right) \bar{n}_{j} \zeta_{k i}\right]\right. \\
& +R_{k j} \zeta_{i j}+R_{i j} \zeta_{k j}-\left(R_{j k}+R_{j i}\right) \zeta_{k i} \\
& +\sigma\left(\delta_{k i}-\delta_{j i}\right)\left(R_{k j}+R_{j k}\right)\left(\bar{n}_{k} \bar{n}_{j}+\zeta_{k j}\right) \\
& \left.+\left(\delta_{k i}-\delta_{j i}\right)\left(R_{k j} \bar{n}_{j}+R_{j k} \bar{n}_{k}\right)\right\}
\end{aligned}
$$

The steady-state values of $\bar{n}_{k}$ and $\zeta_{k i}$ have to be determined by solving Eqs. (45) and (46) with the left-hand side set to zero.

Within the augmented mean-field theory the state is not only described in terms of the mean occupations $\bar{n}_{i}$, but also in terms of nontrivial two-particle correlations $\zeta_{k i}$. As a consequence, we cannot only fix the mean total particle number to a value $N$ by requiring

$$
\langle\hat{N}\rangle=\sum_{i} \bar{n}_{i}=N .
$$

Also, the fluctuation of the total particle number can be fixed to a value $\Delta N$,

$$
\left\langle\hat{N}^{2}\right\rangle-\langle\hat{N}\rangle^{2}=\sum_{i j} \zeta_{i j}=\Delta N^{2} .
$$

This includes the choice

$$
\Delta N=0
$$

for a system of sharp particle number. Whereas the mean-field theory was found to be equivalent to a Gaussian ansatz for the density operator, we cannot give an analytical expression for the density operator corresponding to the augmented theory.

\section{IDEAL BOSE GASES AND BOSE SELECTION}

In this section we discuss in detail the steady state of noninteracting bosonic quantum gases. Let us first recapitulate the case of thermodynamic equilibrium.

\section{A. Equilibrium and Bose condensation}

Under equilibrium conditions, where the rates obey the condition (20), the mean-field equations of motion (31) with $\sigma=1$ for bosons are solved by a steady state characterized by the mean occupations

$$
\bar{n}_{i}=\frac{1}{e^{\beta\left(E_{i}-\mu\right)}-1},
$$

corresponding to Eq. (33) with $\eta_{i}=\beta\left(E_{i}-\mu\right)$. For this solution the right-hand side of Eq. (31) vanishes term by term, indicating detailed balance. The occupation numbers (50) obtained from the non-number-conserving mean-field theory correspond to the exact grand-canonical mean occupations [67] and provide a good approximation also for the canonical ensemble with sharp particle number $N$. In the latter case, the chemical potential has to be chosen such that

$$
\sum_{i} \bar{n}_{i}=N
$$

Assuming the states of the system to be labeled such that

$$
E_{0}<E_{1} \leqslant E_{2} \leqslant \cdots,
$$

meaningful positive occupation numbers correspond to values of the chemical potential below the ground-state energy, $\mu<$ $E_{0}$. The chemical potential increases either when $\beta$ is increased at fixed $N$ or when $N$ is increased at fixed $\beta$.

When in a system of finite extent, with discrete energies $E_{i}$, the particle number $N$ is increased at fixed $\beta$, the chemical potential will eventually approach the ground-state energy so that $E_{0}-\mu \ll E_{1}-E_{0}$. Once this happens at a characteristic particle number $N^{*}$ specified below, the mean occupations of the excited states can be approximated by

$$
\bar{n}_{i} \simeq \frac{1}{e^{\beta\left(E_{i}-E_{0}\right)}-1} \quad \text { for } \quad i \geqslant 1 .
$$

Thus, for $N \gg N^{*}$ the occupations of excited states become independent of $\mu$ (therefore also of $N$ ) and saturate. The occupation of the single-particle ground-state still depends on the chemical potential; assuming $\beta\left(E_{0}-\mu\right) \ll 1$, one finds

$$
\bar{n}_{0} \simeq \frac{1}{\beta\left(E_{0}-\mu\right)} \equiv N_{0},
$$

with

$$
N_{0} \simeq N-\sum_{i \geqslant 1} \frac{1}{e^{\beta\left(E_{i}-E_{0}\right)}-1}
$$

such that $\mu \simeq E_{0}-T / N_{0}$. All particles that cannot be "accommodated" in the excited states will occupy the ground state. This is the phenomenon of Bose-Einstein condensation (or, strictly speaking, its finite size precursor).

In a finite system Bose-Einstein condensation is a crossover, occurring when $N$ becomes comparable to the characteristic value $N^{*}$, which is directly given by the depletion of the condensate,

$$
N^{*}=\sum_{i \geqslant 1} \frac{1}{e^{\beta\left(E_{i}-E_{0}\right)}-1} .
$$

In the thermodynamic limit, defined by taking particle number $N$ and volume $V$ to infinity while holding the density $n=N / V$ at a constant finite value, Bose condensation is a sharp phase transition. At a critical density $n_{c}=N^{*} / V$, the occupation of the ground state becomes macroscopic and the ratio $N_{0} / N$, the condensate fraction, assumes a nonzero value. At the transition $E_{0}-\mu=T / N_{0}$ becomes zero. However, Bose condensation does not necessarily survive the thermodynamic limit. For a homogeneous Bose gas of spatial dimensionality $D \leqslant 2$, the ratio $N^{*} / V$ diverges in the thermodynamic limit due to large occupations of low-energy states, so that no phase transition exists. In this case Bose condensation can still be observed as a crossover in systems of finite size. This is illustrated in Fig. 4(a), where we plot the mean occupations of a bosonic one-dimensional tight-binding chain of $M=20$ sites versus the particle number $N$. In this system $M$ plays the role of a dimensionless volume $V$ so that the density is given by the dimensionless filling factor $n=N / M$. One can observe a sharp crossover: For $N>N^{*}$ the occupations of the excited states saturate so that newly added particles will all become part of the condensate in the ground state, as described by Eqs. (53), (54), and (55). 


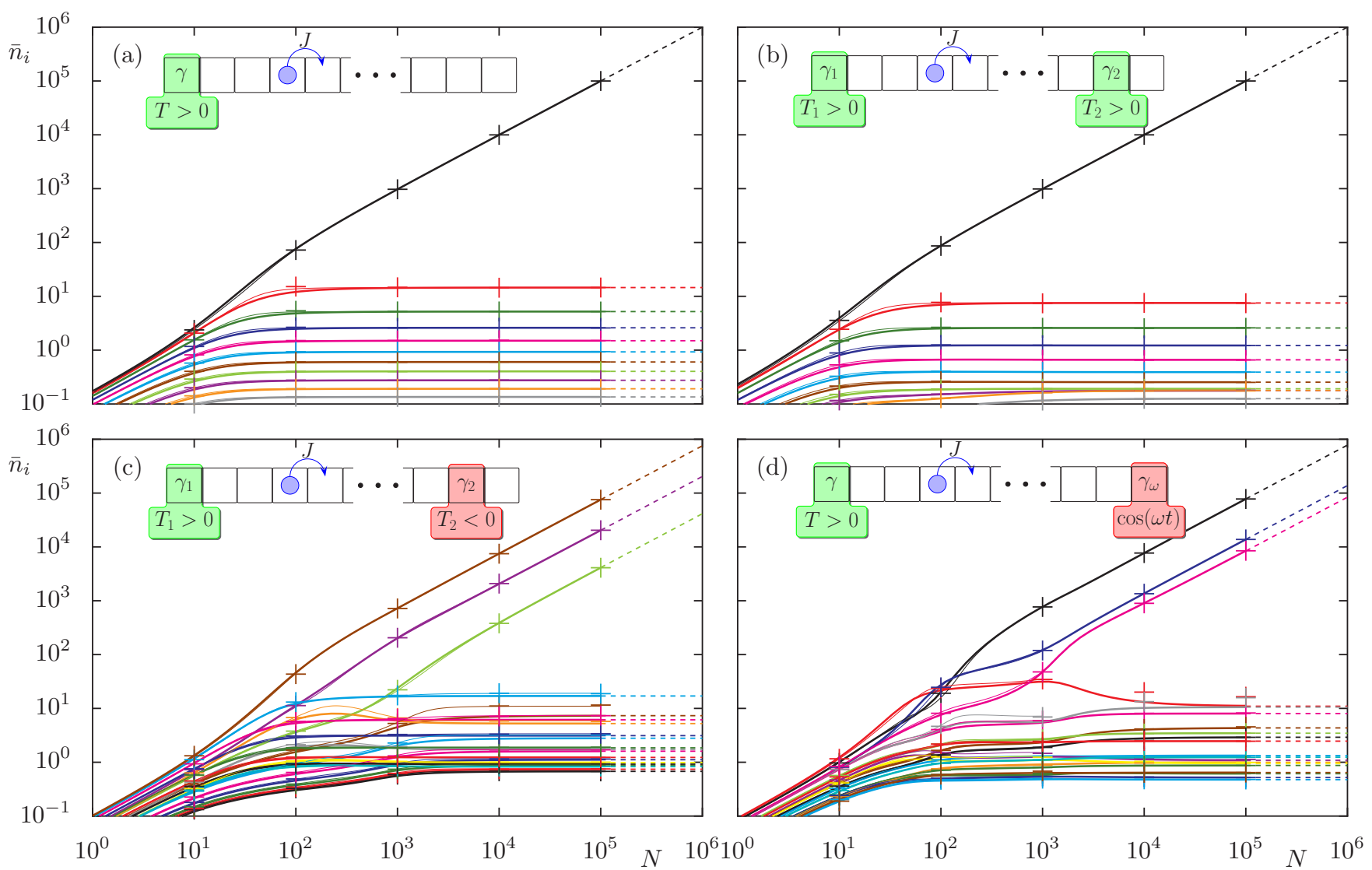

FIG. 4. (Color online) Mean occupations versus total number of bosons for the steady state of a tight-binding chain of $M=20$ sites and tunneling parameter $J>0$. The data are obtained from mean-field theory (thick solid lines), asymptotic mean-field theory (dashed lines), augmented mean-field theory (thin solid lines), and exact Monte Carlo simulations (crosses). (a) Equilibrium situation; the chain is coupled to one bath of temperature $T=1 \mathrm{~J}$. (b) The chain is driven away from equilibrium by two heat baths of different positive temperature $\left(T_{1}=1 \mathrm{~J}\right.$ and $T_{2}=0.5 \mathrm{~J}$ ), coupled to the first and the next to last site with $\gamma_{1}=\gamma_{2}$. (c) Same as in (b), but now the second bath is population inverted and described by the negative temperature $T_{2}=-J$. The color code is the same as in panels (a) and (b), where the occupations decrease with increasing energy. (d) The chain is driven away from equilibrium by a periodic potential modulation at the last site with amplitude $\gamma_{\omega}=2.3 \mathrm{~J}$ and frequency $\hbar \omega=1.5 \mathrm{~J}$. The Floquet states are colored like the stationary states (a)-(c) from which they evolve adiabatically when the driving is switched on (see Fig. 14).

\section{B. Driven-dissipative Bose gas and Bose selection}

The other panels of Fig. 4 show the mean occupations $\bar{n}_{i}$ versus $N$ for situations where the tight-binding chain is driven into a steady state far from equilibrium, either by coupling it to a second bath of different temperature or by time-periodic forcing (see Sec. II D). In each of these panels, we can again identify a sharp crossover. When the particle number $N$ reaches a characteristic value $N^{*}$, many occupations saturate as in equilibrium. However, as a striking effect, newly added particles can now occupy a whole group of states [Figs. 4(c) and 4(d)], with constant relative occupations among these states. These selected states take over the role played by the condensate mode in equilibrium. This phenomenon has been termed Bose selection [23]. It turns out to be the generic behavior in the ultradegenerate regime of large density at fixed finite system size.

As becomes apparent from Fig. 4, we can distinguish two scenarios. In the first, a single state becomes selected. This includes the case of equilibrium Bose condensation depicted in panel (a), but also the nonequilibrium situation shown in panel (b), where a Bose gas is driven out of equilibrium by the coupling to two heat baths of different positive temperature. In the second scenario, multiple states become selected, as can be seen in panels (c) and (d), corresponding to situations where a system is driven out of equilibrium by an additional population-inverted bath of negative temperature or by periodic forcing. As we see in the following, the essential difference between both scenarios is that in the situations (a) and (b) the notion of the single-particle ground state is still meaningful. In panel (b) both baths favor larger occupations in states of lower energy and thus the largest occupation occurs in the ground state. This is not the case anymore for the situations (c) and (d). The population-inverted negative-temperature bath of the system of panel (c) favors larger occupations in states of higher energy counteracting the effect of the positive-temperature bath. For the periodically driven system of panel (d), the quasienergies of the single-particle Floquet states are determined modulo $\hbar \omega$ only, so that a ground state is not even defined.

Within the scenario of having multiple selected states we can, furthermore, distinguish two possibilities. For that purpose we have to consider systems of a large number of states 


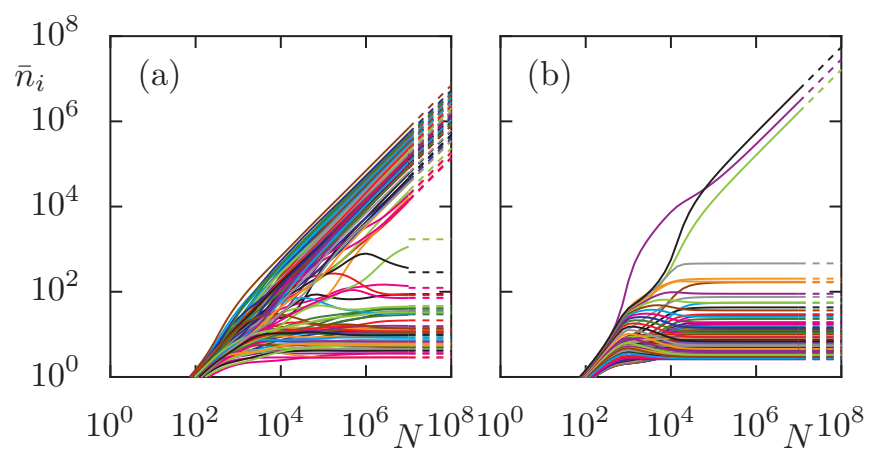

FIG. 5. (Color online) Mean occupations versus total number of bosons for (a) one realization of the random-rate model with $M=$ 100 states and (b) a tight-binding chain of $M=100$ sites coupled to two heat baths, namely one with temperature $T_{1}=10 \mathrm{~J}$ at the first site and a population-inverted bath described by the negative temperature $T_{2}=-10 \mathrm{~J}$ at the fifth-to-last site, with equal coupling strength, $\gamma_{1}=\gamma_{2}$ [see inset of Fig. 4(c)].

$M$. In Fig. 5 we plot the mean occupations for two systems with $M=100$ states. Panel (a) corresponds to one realization of the random-rate model and panel (b) is obtained for a tight-binding chain coupled to a second population-inverted bath like in Fig. 4(c). For the random-rate model (a) the number of selected states $M_{S}$ is of the order of the system size $M$, roughly half of the states become selected for sufficiently large $N$. This implies that none of the selected states acquires a macroscopic occupation of the order of the total particle number. For the tight-binding chain (b) we find that the number of selected states $M_{S}$ is still of the order of one; namely, three states are selected. As a consequence, each selected state acquires a macroscopic occupation of the order of the total particle number and hosts a Bose condensate. This corresponds to fragmented Bose condensation, ${ }^{4}$ which is therefore a generic situations for driven Bose gas, unlike in equilibrium where this requires a rare ground-state degeneracy. Thus, all in all, we can distinguish three generic types of Bose selection occurring in the ultradegenerate regime of driven-dissipative ideal Bose gases: standard Bose condensation where a single state acquires a macroscopic occupation, fragmented Bose condensation where a small number (of order one) of selected states each acquires macroscopic occupation, and the selection of a large number of states with nonextensive individual occupations that together attract most particles of the system.

In the following we provide a theory for Bose selection based on mean-field theory in the asymptotic limit of large $N$.

\footnotetext{
${ }^{4}$ Note that the system does not feature a single condensate in a state being a coherent superposition of the highly occupied selected modes, but independent condensates in each mode. Namely, according to the Penrose-Onsager criterion Bose-Einstein condensation is defined by a macroscopic eigenvalue of the single-particle density matrix $\left\langle a_{i}^{\dagger} a_{j}\right\rangle$ [68]. In the situation discussed here, the off-diagonal elements of $\left\langle a_{i}^{\dagger} a_{j}\right\rangle$ are negligible as a consequence of the weak coupling to the bath. Therefore, each macroscopic mean occupation $\bar{n}_{i}=\left\langle a_{i}^{\dagger} a_{i}\right\rangle$ corresponds to a macroscopic eigenvalue of the single-particle density matrix and an independent Bose condensate.
}

It can be viewed as a generalization of the Eqs. (53), (54) and (55) describing equilibrium Bose condensation to the case of driven-dissipative ideal Bose gases. Later, also effects beyond mean field will be discussed in terms of the augmented meanfield theory.

\section{Asymptotic mean-field theory}

A theoretical description of Bose selection can be based on mean-field theory, given by Eq. (31) with $\sigma=1$. For the steady state this equation reads

$$
0=\sum_{j}\left[R_{i j} \bar{n}_{j}\left(1+\bar{n}_{i}\right)-R_{j i} \bar{n}_{i}\left(1+\bar{n}_{j}\right)\right]
$$

for all $i$. Since Bose selection occurs in the asymptotic limit of large densities, it appears natural to approximate

$$
1+\bar{n}_{k} \approx \bar{n}_{k}
$$

in this equation. One then obtains the equations ${ }^{5}$

$$
0=\bar{n}_{i} \sum_{j}\left(R_{i j}-R_{j i}\right) \bar{n}_{j}=\bar{n}_{i} \sum_{j} A_{i j} \bar{n}_{j} .
$$

One can immediately see that some of the mean occupations $\bar{n}_{i}$ have to vanish on this level of approximation. Namely, if we assume that a subset $\mathcal{S}$ of single-particle states possesses nonzero occupations, these states have to obey the linear equations

$$
0=\sum_{j \in \mathcal{S}} A_{i j} \bar{n}_{j}, \quad i \in \mathcal{S},
$$

which directly follow from Eq. (59). However, without fine tuning of the skew-symmetric asymmetry matrix $A_{i j}=-A_{j i}$, these equations have a solution only if $\mathcal{S}$ contains an odd number of states (since a skew-symmetric matrix generically possesses an eigenvalue zero only when acting in an odddimensional space). Moreover, even if a formal solution can be found for a certain set $\mathcal{S}$, it is not guaranteed that this solution will correspond to physically meaningful solutions, where all occupation numbers are non-negative. Both conditions constrain the set $\mathcal{S}$, so that, generically, it will not contain all states. Those states contained in the (yet to be determined) set $\mathcal{S}$ correspond to the Bose-selected states.

In order to compute the occupations of the nonselected states, we have to include another level of approximation. For that purpose we use that the occupation of a nonselected state is determined predominantly by transitions from or into selected states. The large occupations of the selected states enhances the corresponding rates with respect to the rates for transitions from or into other nonselected states. Thus, neglecting transitions among nonselected states and still

\footnotetext{
${ }^{5}$ It is interesting to note that these equations correspond to the conservative Lotka-Volterra equations $\dot{\bar{n}}_{i}=\bar{n}_{i} \sum_{j} A_{i j} \bar{n}_{j}$ as they are used to model population dynamics. Indeed, for fully connected rate matrices, the selected states correspond directly to those species that will not be extinct, but survive $[46,47,69]$. Differences appear, however, for not fully connected rate matrices, as discussed at the end of Sec. IV I.
} 
assuming $n_{j}+1 \approx n_{j} \forall j \in \mathcal{S}$, from Eqs. (57) for nonselected states $i$ we obtain

$$
\bar{n}_{i}=\frac{1}{g_{i}-1} \quad \text { with } \quad g_{i}=\frac{\sum_{j \in \mathcal{S}} R_{j i} \bar{n}_{j}}{\sum_{j \in \mathcal{S}} R_{i j} \bar{n}_{j}}, \quad i \notin \mathcal{S} .
$$

This approximation is reminiscent of the Bogoliubov approximation [70] for the weakly interacting Bose gas, where interactions among noncondensed particles are neglected.

The set $\mathcal{S}$ has to be chosen such that physically meaningful occupations

$$
\bar{n}_{i} \geqslant 0
$$

are obtained for all $i$ [i.e., both for the selected states, whose relative occupations are determined by Eq. (60), and for the nonselected states, with the occupations given by Eq. (61)]. We prove in the following Sec. IV E that there exists a unique set $\mathcal{S}$ for which condition (62) is fulfilled. Thus, the problem to be solved does not simply consist of solving Eqs. (60) and (61) for a given set $\mathcal{S}$. It is rather the task of finding both the occupations $\bar{n}_{i}$ and the set $\mathcal{S}$, for which the relations (60), (61), and (62) are fulfilled.

By identifying the states of the set $\mathcal{S}$ with the selected states, we can now explain the major features of the results presented in Fig. 4. One observation is that for large $N$ the relative occupations among the selected states become independent of $N$. This is explained by the fact that these relative occupations are determined by the set of linear Eqs. (60), which does not depend on $N$. A second observation is that the occupations of the nonselected states saturate in the limit of large $N$. Such a behavior is predicted by Eq. (61), where the $g_{i}$ are determined by the $N$-independent relative occupations of the selected states. This implies also that the total occupation of the selected states,

$$
N_{S}=\sum_{i \in \mathcal{S}} \bar{n}_{i}=N-\sum_{i \notin \mathcal{S}} \frac{1}{g_{i}-1},
$$

grows linearly with $N$. Finally, we can estimate the characteristic particle number $N^{*}$ at which the crossover to Bose selection occurs to be given by the depletion of the selected states, i.e., by the total number of particles in nonselected states,

$$
N^{*}=\sum_{i \notin \mathcal{S}} \frac{1}{g_{i}-1} .
$$

The set of selected states is determined completely by the rate-asymmetry matrix $A_{i j}$. Namely, this matrix determines not only the relative occupations among the selected states via Eqs. (60), but also the sign of the occupations (61) of the nonselected states, which have to be positive. The latter can be seen by writing Eq. (61) as

$$
\bar{n}_{i}=\frac{1}{g_{i}-1}=-\frac{\sum_{j \in \mathcal{S}} R_{i j} \bar{n}_{j}}{\sum_{j \in \mathcal{S}} A_{i j} \bar{n}_{j}}, \quad i \notin \mathcal{S} .
$$

Here the numerator is always positive, since both the rates $R_{i j}$ and the occupations $\bar{n}_{j}$ are positive, and the sign of the denominator is determined by $A_{i j}$, since it depends on the relative occupations among the selected states, which are determined by $A_{i j}$ via Eqs. (60). The fact that the rate-asymmetry matrix $A_{i j}$, given by Eq. (8) or by Eq. (9), does not depend on the bath temperature(s) implies that the set of selected states $\mathcal{S}$ also does not depend on the bath temperature(s). However, the occupations (61) of the nonselected states are temperature dependent, as $R_{i j}$ appears on the right-hand side of Eq. (65). This implies that both the total number of particles in selected states $N_{S}$ [Eq. (63)] as well as the characteristic particle number $N^{*}$ [Eq. (64)] at which Bose selection sets in depend on the bath temperature(s).

Finding the set of selected states $\mathcal{S}$ is generally a nontrivial problem. A brute-force algorithm would go through all possible sets containing an odd number of single-particle states, whose number grows exponentially with the number of modes $M$, until the desired set $\mathcal{S}$ is found. An efficient algorithm for finding $\mathcal{S}$ is presented in Sec. IV G below. Already the question of how many states will be selected is not straightforward to answer, apart from the fact that (without fine tuning) it is always an odd number.

A special case is the scenario of having a single selected state $k$, corresponding to standard Bose condensation. Here the occupations of the nonselected states (61) reduce to the simple expression

$$
\bar{n}_{i}=\frac{1}{R_{k i} / R_{i k}-1}, \quad i \neq k .
$$

The fact that these occupations must be positive reveals that this scenario occurs when the state $k$ is ground-state-like in the sense that for all states $i$ the rate $R_{k i}$ from $i$ to $k$ is always larger than the backward rate $R_{i k}$,

$$
R_{k i}-R_{i k}=A_{k i}>0 \quad \forall i \neq k .
$$

The term "ground-state-like" refers to the situation of thermal equilibrium, where the relation (20) implies that the condition (67) is fulfilled for $k$ being the ground state. These arguments reveal why we find a single selected state for the tight-binding chain which is driven between two heat baths of different positive temperature [Fig. 4(b)]. In this situation the notion of the single-particle ground state still remains meaningful even away from equilibrium. This is generally different when the system is coupled to a population-inverted bath described by a negative temperature, like in Fig. 4(c), or in a periodically driven system, like in Fig. 4(d). In the former case the condition (67) cannot be expected to hold for $k$ being the ground state and in the latter case the ground state is not even defined (since quasienergies are determined modulo $\hbar \omega$ only).

We can compare our theory to the theory of equilibrium Bose condensation as it was reviewed in Sec. IV A. First of all, we would like to note that the equilibrium situation is contained in our asymptotic mean-field theory as a special case. Namely, the equilibrium expression (53) for the excited-state occupations is reproduced, when the relation (20) is plugged into Eq. (66). Generally, our Eq. (61) generalizes Eq. (53); likewise, Eqs. (63) and (64) are generalizations of Eqs. (55) and (56), respectively. However, the fact that the relative occupations among the selected states and, even more, also the set $\mathcal{S}$ of selected states have to be determined adds an additional layer of complexity to the theory of nonequilibrium Bose selection. 


\section{Systematic high-density expansion}

In this section we show that the asymptotic mean-field theory described in the previous subsection corresponds to the leading orders of a systematic expansion in the inverse total particle number $N^{-1}$. This implies that it correctly captures the mean-field result in the limit of large $N$.

Let us expand the mean occupations as a series in powers of the inverse particle number $N^{-1}$,

$$
\bar{n}_{i}=N v_{i}+v_{i}^{(1)}+N^{-1} v_{i}^{(2)}+N^{-2} v_{i}^{(3)}+\cdots,
$$

and require

$$
\sum_{i} v_{i}=1, \quad \sum_{i} v_{i}^{(r)}=0,
$$

for the leading order as well as for the corrections of order $r \geqslant 1$. These requirements ensure that the mean total particle number is given by $N$, when the series is truncated after a certain order $r$. Such an expansion is equivalent to an expansion in the inverse particle density $n^{-1}=M / N$. We can now plug the ansatz (68) into the mean-field Eqs. (57),

$$
\begin{aligned}
0= & v_{i} \sum_{j} A_{i j} v_{j} \\
& +\frac{1}{N} \sum_{j}\left[R_{i j} v_{j}-R_{j i} v_{i}+A_{i j}\left(v_{i} v_{j}^{(1)}+v_{i}^{(1)} v_{j}\right)\right] \\
& +\frac{1}{N^{2}} \sum_{j}\left[R_{i j} v_{j}^{(1)}-R_{j i} v_{i}^{(1)}\right. \\
& \left.+A_{i j}\left(v_{i}^{(2)} v_{j}+v_{i}^{(1)} v_{j}^{(1)}+v_{i} v_{j}^{(2)}\right)\right]+O\left(\frac{1}{N^{3}}\right)
\end{aligned}
$$

and ask that all terms that correspond to the same power of $N$ vanish independently. In this way we get a hierarchy of equations determining the coefficients of the expansion (68) order by order.

Collecting the terms of the leading order gives rise to a set of equations for the leading coefficients $v_{i}$. These equations take the form of Eqs. (59), but with $\bar{n}_{i}$ replaced with $v_{i}$,

$$
0=v_{i} \sum_{j} A_{i j} v_{j} .
$$

Repeating the arguments of the previous section we see that the leading-order coefficient is nonzero only for a (yet to be determined) set of selected states $\mathcal{S}$, so that

$$
v_{i}=0, \quad i \notin \mathcal{S},
$$

and

$$
0=\sum_{j \in \mathcal{S}} A_{i j} v_{j}, \quad i \in \mathcal{S} .
$$

The next order determines the coefficients $v_{i}^{(1)}$. Thanks to Eq. (72) the coefficients of the nonselected states are not coupled to each other and depend on the leading-order occupations of the selected states only, so that we arrive at the simple expression

$$
v_{i}^{(1)}=-\frac{\sum_{j \in \mathcal{S}} R_{i j} v_{j}}{\sum_{j \in \mathcal{S}} A_{i j} v_{j}}, \quad i \notin \mathcal{S} .
$$

This expression directly corresponds to Eq. (61), but with $\bar{n}_{i}$ replaced with $v_{i}$ for the selected and by $v_{i}^{(1)}$ for the nonselected states. The leading corrections to the occupations of the selected states appear in the same order and can be determined by solving the linear equations

$$
\begin{aligned}
\sum_{j \in \mathcal{S}} A_{i j} v_{i} v_{j}^{(1)}= & \sum_{j \in \mathcal{S}}\left(R_{j i} v_{i}-R_{i j} v_{j}\right) \\
& +v_{i} \sum_{j \notin \mathcal{S}}\left(R_{j i}-A_{i j} v_{j}^{(1)}\right), \quad i \in \mathcal{S},
\end{aligned}
$$

where we used $0=\sum_{j \in \mathcal{S}} A_{i j} v_{j}$ [Eqs. (71) and (72)] and taking Eq. (69) for $r=1$ into account. Higher orders in the expansion (70) can become relevant when some rates vanish, as discussed in Sec. IV H.

Truncating the $1 / N$ expansion after the first order, one obtains

$$
\bar{n}_{i} \simeq \begin{cases}v_{i} N+v_{i}^{(1)} & \text { for } i \in \mathcal{S}, \\ v_{i}^{(1)} & \text { for } i \notin \mathcal{S} .\end{cases}
$$

However, asymptotically in the limit of large $N$, it will be sufficient to take into account only the leading contributions, so that the mean occupations can be approximated as

$$
\bar{n}_{i} \simeq \begin{cases}v_{i} N & \text { for } i \in \mathcal{S}, \\ v_{i}^{(1)} & \text { for } i \notin \mathcal{S} .\end{cases}
$$

This corresponds to the approximation of the previous section, apart from the slight difference that, previously, we normalized the total occupation of the selected states $N_{S}$ to the firstorder result $N_{s}^{(1)}=\sum_{i \in \mathcal{S}}\left[v_{i} N+v_{i}^{(1)}\right]=N-\sum_{i \neq \mathcal{S}} v_{i}^{(1)}$. This is implicit in Eq. (63) and corresponds to the approximation

$$
\bar{n}_{i} \simeq \begin{cases}v_{i} N_{s}^{(1)} & \text { for } i \in \mathcal{S}, \\ v_{i}^{(1)} & \text { for } i \notin \mathcal{S} .\end{cases}
$$

This normalization, which for finite $N$ takes care of the fact that the leading contributions to the occupations of the selected and the nonselected states stem from different orders, is thus a compromise between Eq. (76) and Eq. (77). For large but finite $N$ it is better than Eq. (77), since it produces the correct total particle number, but it does not require to compute corrections $v_{i}^{(1)}$ for the selected states that enter Eq. (76). Therefore, we use Eq. (78), corresponding to the asymptotic theory as it was presented in the previous section, in the following. In the asymptotic limit $N \rightarrow \infty$ all three expressions (76), (77), and (78) are, of course, equivalent.

The requirement of having a positive particle number in the asymptotic limit of large $N$ is given by

$$
\begin{array}{ll}
v_{i}>0 & \text { for } i \in \mathcal{S}, \\
v_{i}^{(1)}>0 & \text { for } i \notin \mathcal{S} .
\end{array}
$$

In order to find a compact formulation of finding an asymptotic solution obeying this condition it is convenient to introduce the numbers $\mu_{i}=\sum_{j} A_{i j} v_{j}$. According to Eq. (73) they vanish for $i \in \mathcal{S}$, while Eq. (74) tells us that they should be negative to ensure positive occupations of the nonselected states. The problem of finding an asymptotic mean-field solution can, 
therefore, be reduced to the problem of finding a set $\mathcal{S}$ of selected states and numbers $v_{i}$ and $\mu_{i}$ such that [23]

$$
\mu_{i}=\sum_{j} A_{i j} v_{j} \text { with }\left\{\begin{array}{l}
v_{i}>0 \text { and } \mu_{i}=0 \text { for } i \in \mathcal{S}, \\
v_{i}=0 \text { and } \mu_{i}<0 \text { for } i \notin \mathcal{S} .
\end{array}\right.
$$

The nongeneric situation with $v_{i}=\mu_{i}=0$ for some $i$ corresponds to transitions, which we discuss in the next section. Before we prove that a unique set $\mathcal{S}$ obeying the relations (80) exists, let us point out that these relations are valid only in the case of fully connected rate matrices. If we allow for zero rates $R_{i j}=0$, the set of selected states is not determined by the conditions (80) anymore, as we discuss in Sec. IV I below.

It is interesting to note that the conditions (80) that determine the selected states are equivalent to those determining the surviving species under the dynamics of the Lotka-Volterra equations given in footnote 5 [46,47]. Differences appear for non-fully-connected rate matrices (see discussion at the end of Sec. IV I).

\section{E. Existence and uniqueness of the set of selected states}

In this section we provide a proof for the uniqueness and the existence of the set of selected states for fully connected rate matrices (which we repeat for completeness from the Supplemental Material of Ref. [23].) In the following we use the vector and matrix notation, with $\boldsymbol{v}$ and $\boldsymbol{\mu}$ denoting the vectors with elements $v_{i}$ and $\mu_{i}$, respectively, and $R$ and $A$ denoting the rate matrix and the rate-asymmetry matrix with elements $R_{i j}$ and $A_{i j}$, respectively. Let us, furthermore, decompose $A$ like

$$
A=\left(\begin{array}{c|c}
A^{\mathcal{S}} & A^{\mathcal{S}} \overline{\mathcal{S}} \\
\hline A^{\mathcal{S}} & A^{\mathcal{S}}
\end{array}\right)
$$

wherein the submatrix $A^{\mathcal{S}}=\left\{A_{i j}\right\}_{i, j \in \mathcal{S}}$ denotes the rate asymmetries among selected states, $A^{\overline{\mathcal{S}} \mathcal{S}}=-\left(A^{\mathcal{S} \overline{\mathcal{S}}}\right)^{T}=$ $\left\{A_{i j}\right\}_{i \notin \mathcal{S}, j \in \mathcal{S}}$ the rate asymmetries among nonselected and selected states, and $A^{\overline{\mathcal{S}}}=\left\{A_{i j}\right\}_{i, j \notin \mathcal{S}}$ the rate asymmetries among nonselected states. The conditions (80) with $i \in \mathcal{S}$ require us to determine $\mathcal{S}$ such that $A^{\mathcal{S}}$ has a vanishing eigenvalue. Skewsymmetric matrices generically have a vanishing eigenvalue only if their dimension is odd. As the square submatrix $A^{\mathcal{S}}$ of $A$ is still skew symmetric, we can immediately conclude that the number $M_{S}$ of Bose selected states is odd. The conditions (80) stipulate, furthermore, that the corresponding eigenvector $v_{i}, i \in \mathcal{S}$ has positive components. Finally, the conditions for $i \notin \mathcal{S}$ tell us that this eigenvector should result in a vector with nonpositive components when it is multiplied with the submatrix $A^{\overline{\mathcal{S}} \mathcal{S}}$.

We now prove the uniqueness of the set $\mathcal{S}$. Assume first that there exist two different sets $\mathcal{S}_{1}$ and $\mathcal{S}_{2}$, both leading to physical solutions $\boldsymbol{v}_{1}$ and $\boldsymbol{v}_{2}$ with $\mu_{1}=A \boldsymbol{v}_{1}$ and $\mu_{2}=A \boldsymbol{v}_{2}$ obeying Eq. (80). Using

$$
\begin{aligned}
\boldsymbol{v}_{2}^{T} \boldsymbol{\mu}_{1} & =\boldsymbol{v}_{2}^{T} A \boldsymbol{v}_{1}=\left(\boldsymbol{v}_{2}^{T} A \boldsymbol{v}_{1}\right)^{T}=\boldsymbol{v}_{1}^{T} A^{T} \boldsymbol{v}_{2}=-\boldsymbol{v}_{1}^{T} A \boldsymbol{v}_{2} \\
& =-\boldsymbol{v}_{1}^{T} \boldsymbol{\mu}_{2},
\end{aligned}
$$

it then follows from Eq. (80) that

$$
0 \geqslant \boldsymbol{v}_{2}^{T} \boldsymbol{\mu}_{1}=-\boldsymbol{v}_{1}^{T} \boldsymbol{\mu}_{2} \geqslant 0 .
$$

This requires that both $\boldsymbol{v}_{2}^{T} \boldsymbol{\mu}_{1}=0$ and $\boldsymbol{v}_{1}^{T} \boldsymbol{\mu}_{2}=0$, such that $\mathcal{S}_{2} \subset \mathcal{S}_{1}$ and $\mathcal{S}_{1} \subset \mathcal{S}_{2}$, leading us to conclude that $\mathcal{S}_{1}=\mathcal{S}_{2} \equiv$ $\mathcal{S}$. Given the set $\mathcal{S}$, the homogeneous linear system for $\boldsymbol{v}$ generically has a single solution only. Therefore, the solution to the generic steady-state problem has to be unique.

In order to prove the existence of the set $\mathcal{S}$, we now restrict $\mathcal{S}$ to sets comprising an odd number $M_{S}$ of states, according to the generic conditions described above. Each choice of $\mathcal{S}$ gives rise to a (possibly nonphysical) solution $\boldsymbol{v}_{\mathcal{S}}$ with $\boldsymbol{\mu}_{\mathcal{S}}=A \boldsymbol{v}_{\mathcal{S}}$. The vector of signs $\sigma$ with

$$
\begin{aligned}
& \sigma_{i}=\operatorname{sgn}\left(v_{i}\right) \text { if } i \in \mathcal{S}, \\
& \sigma_{i}=-\operatorname{sgn}\left(\mu_{i}\right) \text { if } i \notin \mathcal{S},
\end{aligned}
$$

distinguishes physical solutions $\left(\sigma_{i}=1\right.$ for all $\left.i\right)$ from nonphysical solutions. Here we fix an overall sign due to the orientation of the vector $\boldsymbol{v}_{\mathcal{S}}$ by the convention $\sigma_{1}=1$. Now we observe the following. (i) Cycling through all odd-numbered subsets $\mathcal{S}$, each possible vector $\sigma$ occurs at most once. Namely, if $\mathcal{S}_{1}$ and $\mathcal{S}_{2}$ gave rise to the same vector $\sigma$, then the modified rate-imbalance matrix $\tilde{A}_{i j}=\sigma_{i} A_{i j} \sigma_{j}$ had two physical solutions with different selected sets $\mathcal{S}_{1}$ and $\mathcal{S}_{2}$, in contradiction to the previously established uniqueness of the solutions. (ii) The number $2^{M-1}$ of possible vectors $\sigma$ equals the number $\sum_{M_{S}=1,3, \ldots}\left(\begin{array}{c}M \\ M_{S}\end{array}\right)=2^{M-1}$ of possible sets $\mathcal{S}$. Therefore, each vector $\sigma$ occurs once. In particular, this includes the vector with $\sigma_{i}=1$ for all $i$, leading to the solution with positive macroscopic and microscopic occupations. This guarantees the existence of a physical solution.

\section{F. Transitions}

In this section we discuss transitions, where the set of selected states $\mathcal{S}$ changes in response to the variation of a parameter $p$. Examples for such transitions can be observed in Fig. 6. This figure shows the mean occupations versus the parameter $p$ for a model defined by the superposition of two random-rate matrices $R^{(1)}$ and $R^{(2)}$, with the relative weight controlled by $p, R(p)=(1-p) R^{(1)}+p R^{(2)}$. One can see that in a transition two states are exchanged between the set of selected states and the set of nonselected states, such that the number of selected states is odd before and after the transition. Approaching a transition from the left, the transition is found to be triggered by a state $i^{<}$. This state $i^{<}$can either be a selected state whose occupation drops until it becomes nonselected at the transition (case I) or a nonselected state whose occupation increases until it becomes selected at the transition (case II). Furthermore, one can observe that at the transition a second state $i^{>}$becomes involved abruptly that changes from the selected to nonselected (case A) or vice versa (case B). When approaching the transition from the right, the states $i^{<}$and $i^{>}$ change their role, so that the former partner state $i^{>}$plays the role of the triggering state.

The four combinations of cases I or II and A or B define four generic types of transitions that are depicted in Fig. 7. Type (I,A) and type (II,B), where the number $M_{\mathcal{S}}$ of selected states is lowered or raised by two, respectively, transform into each other when the transition is passed in opposite direction. Therefore, they form one class. In type (II,A) transitions, which are triggered by nonselected states from both side, and 


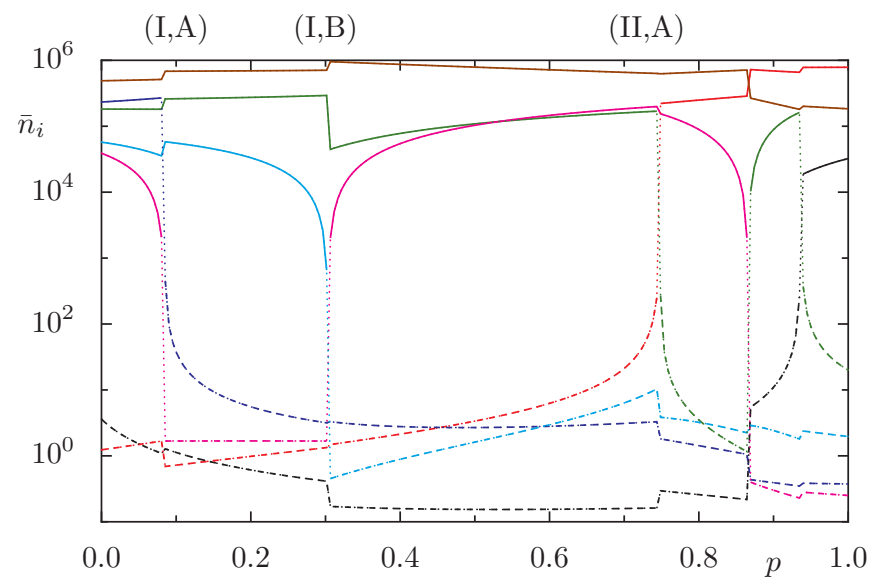

FIG. 6. (Color online) Mean occupations in response to the variation of a dimensionless parameter $p$, for a small system of $N=10^{6}$ bosons on $M=7$ states for the random-rate model. The rate matrix $R(p)$ is a superposition of two independently drawn rate matrices $R^{(1)}$ and $R^{(2)}$, with the relative weight controlled by $p, R(p)=(1-p) R^{(1)}+p R^{(2)}$. The results are obtained using mean-field theory (dotted lines) and asymptotic theory (solid lines for selected states and dashed lines for nonselected states). Each color refers to a specific state. At each transition two states are exchanged between the sets of selected and nonselected states.

type (I,B) transitions, which are triggered from selected states from both sides, the number $M_{\mathcal{S}}$ of selected states does not change. They define two distinct classes, since they cannot be transformed into each other.
1
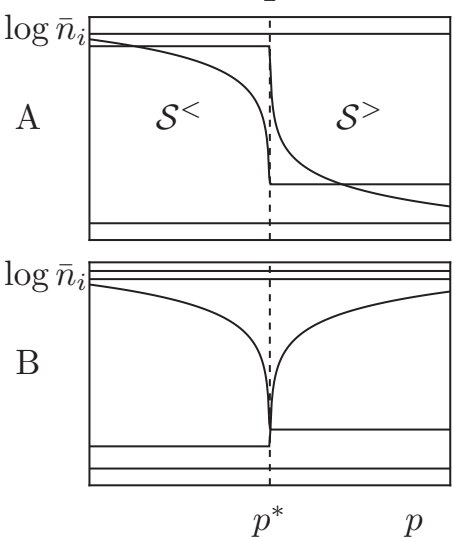

II
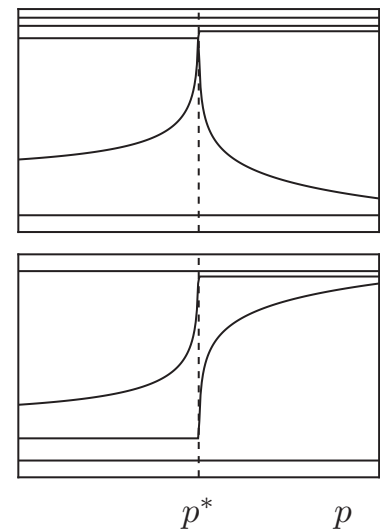

FIG. 7. Four generic types of transitions, where the set of selected states changes from $\mathcal{S}=\mathcal{S}^{<}$to $\mathcal{S}=\mathcal{S}^{>}$when a parameter $p$ reaches a critical value $p^{*}$. In each transition, two states are exchanged between the sets of selected and nonselected states, so that the number $M_{\mathcal{S}}$ of selected states remains odd. When approaching the transition from the left, it is triggered by a state $i^{<}$, either a selected state whose occupation drops until it becomes nonselected at the transition (case I) or a nonselected state whose occupation increases until it becomes selected at the transition (case II). A second state $i^{>}$becomes involved abruptly at the transition that changes from selected to nonselected (case A) or vice versa (case B). This state plays the role of the triggering state when the transition is approached from the right. Types (I,A) and (II,B) form one class, since they transform into each other when the transition is passed in opposite direction.
These observations based on Fig. 6 turn out to be generic. In the following we describe them within the asymptotic meanfield theory. We have already defined the left triggering state $i^{<}$and its partner state, the right triggering state $i^{>}$. Moreover, let $p^{*}$ be the critical parameter at which the transition occurs and $\mathcal{S}^{<}$and $\mathcal{S}^{>}$be the sets of selected state on the left-hand and the right-hand side of the transition, respectively (Fig. 7). Within the asymptotic theory a transition must occur when the occupation $\bar{n}_{i}$ of a state $i$ would change its sign at a critical parameter $p=p^{*}$. This state $i$ plays the role of the triggering state $i^{<}$. If $i^{<}$is a selected state (before the transition), the transition occurs when $v_{i}<$ drops to zero, so that in zeroth order the occupation of this state becomes zero. In that case the state $i^{<}$can, thus, be viewed as a nonselected state at the transition. If $i^{<}$is a nonselected state, the transition occurs when $\mu_{i}<$ becomes zero, so that in first order the occupation of this state diverges. In that case the state $i^{<}$must, therefore, be viewed as a selected state at the transition. Thus, at the transition $p=p^{*}$, which corresponds to a fine-tuned situation, the set of selected states contains an even number of states and is given by

$$
\mathcal{S}^{*}= \begin{cases}\mathcal{S}^{<} \cup\left\{i^{<}\right\} & \text {if } i^{<} \notin \mathcal{S}^{<}, \\ \mathcal{S}^{<} \backslash\left\{i^{<}\right\} & \text {if } i^{<} \in \mathcal{S}^{<} .\end{cases}
$$

As the number of Bose-selected states has to become odd after the transition, one further state $i^{>}$has to be involved. The set $\mathcal{S}^{*}$ can also be expressed in terms of this partner state,

$$
\mathcal{S}^{*}= \begin{cases}\mathcal{S}^{>} \cup\left\{i^{>}\right\} & \text {if } i^{>} \notin \mathcal{S}^{>}, \\ \mathcal{S}^{>} \backslash\left\{i^{>}\right\} & \text {if } i^{>} \in \mathcal{S}^{>} .\end{cases}
$$

In the following we describe how to determine this partner state in order to find the set $\mathcal{S}^{>}$of selected states on the other side of the transition.

The intricate details of the transition are encoded in the truncated matrix $A^{\mathcal{S}^{*}}$, obtained from $A^{*}=A\left(p^{*}\right)$ by removing all rows and columns corresponding to nonselected states $i \notin \mathcal{S}^{*}$ like in Eq. (81). According to the transition criteria, this matrix has at least one vanishing eigenvalue. As the matrix is even-dimensional and skew-symmetric, its eigenvalues are imaginary and come in pairs of opposite sign. Thus, one eigenvalue of zero implies another one, so that generically the kernel of $A^{\mathcal{S}^{*}}$ will be two-dimensional at the transition. One vector lying in the kernel of $A^{S^{*}}$ is given by the limiting occupations $v_{i}$ as one approaches $p^{*}$ from below. We denote this vector by $\boldsymbol{v}^{<}$(note that this is now truncated to the states of $\mathcal{S}^{*}$ ). Analogously, there is a second vector $\boldsymbol{v}^{>}$from the limiting occupations as one approaches $p^{*}$ from the right, which also lies in the kernel. We now establish a relation between both vectors $\boldsymbol{v}^{<}$and $\boldsymbol{v}^{>}$.

For that purpose, we introduce an interpolating vector $\boldsymbol{v}(a)=a \boldsymbol{v}^{<}+(1-a) \boldsymbol{v}^{\prime}$, where $\boldsymbol{v}^{\prime}$ is the element of the kernel of $A^{*}$ which is orthogonal to $\boldsymbol{v}^{<}$, while $a$ is an interpolation parameter. The occupations of the nonselected states (and their sign) is determined by the vector $\boldsymbol{\mu}$ given by Eq. (80). For the two possible solutions $\boldsymbol{v}^{<}$and $\boldsymbol{v}^{>}$, this vector reads $\boldsymbol{\mu}^{<}=A^{\overline{\mathcal{S}}^{*} \mathcal{S}^{*}} \boldsymbol{v}^{<}$and $\boldsymbol{\mu}^{>}=A^{\overline{\mathcal{S}}^{*} \mathcal{S}^{*}} \boldsymbol{v}^{>}$, respectively. Both vectors are connected by the interpolation $\boldsymbol{\mu}(a)=a \boldsymbol{\mu}^{<}+(1-a) \boldsymbol{\mu}^{\prime}$ with $\boldsymbol{\mu}^{\prime}=A^{\overline{\mathcal{S}}^{*} \mathcal{S}^{*}} \boldsymbol{v}^{\prime}$. Herein $A^{\overline{\mathcal{S}}^{*} \mathcal{S}^{*}}$ is obtained from $A^{*}=A\left(p^{*}\right)$ as described by Eq. (81). Due to the selection criterion Eq. (80), we require physical solutions 


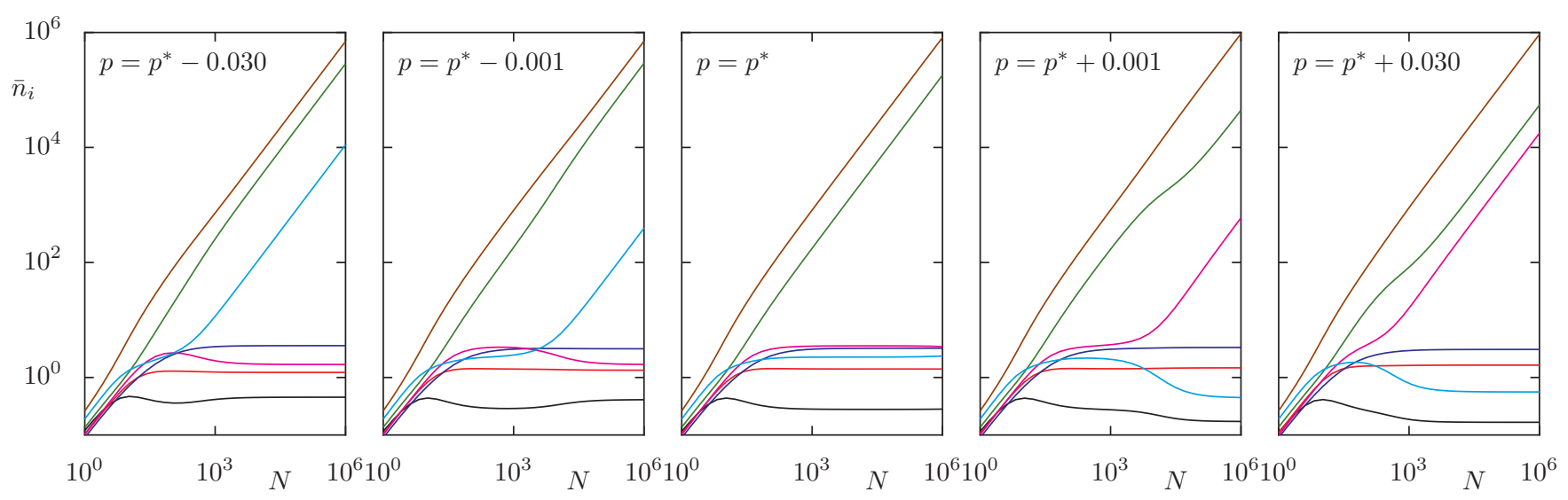

FIG. 8. (Color online) Occupations numbers versus total particle number $N$ close to a transition. The system is described by the same rate matrix $R(p)$ as that of Fig. 6. The parameters $p$ used in the different panels are chosen to be close (or at) the transition labeled (I,B) in Fig. 6, with $p^{*}=0.303179$ denoting the corresponding critical parameter.

$v_{i}(a) \geqslant 0 \forall i \in \mathcal{S}^{*}$ and $\mu_{i}(a) \leqslant 0 \forall i \notin \mathcal{S}^{*}$. Choosing the orientation of $\boldsymbol{v}^{\prime}$ conveniently, this is fulfilled for the finite interval $0<a<a^{>}$. The extremal point $a^{>}$is determined by ramping up $a$ until either an element of $\boldsymbol{v}(a)$ or $\boldsymbol{\mu}(a)$ becomes zero. The index of this element corresponds to the state $i^{>}$ and the extremal point $a^{>}$determines the solution $\boldsymbol{v}^{>}$, via $\boldsymbol{v}\left(a^{>}\right)=\boldsymbol{v}^{>}$.

Exactly at the transition, the interval $0<a<a^{>}$corresponds to physically meaningful solutions with positive occupation numbers. Its extremal points describe the solutions $\boldsymbol{v}^{<}$and $\boldsymbol{v}^{>}$found when approaching the transition from the leftand right-hand side, respectively. The narrower the interval, i.e., the smaller $\Delta a=a^{>}$, the more similar will both solutions $\boldsymbol{v}^{<}$and $\boldsymbol{v}^{>}$be. That means the smaller will be the discontinuous changes in the occupations of the states $i \notin\left\{i^{<}, i^{>}\right\}$that are not directly involved in the transition, as they are visible also in Fig. 6. The width $\Delta a$ associated with a typical transition must, moreover, be expected to shrink with the system size. Namely, each of the $M$ single-particle states of the system provides a constraint that potentially limits this interval, since the number of conditions Eq. (80) proliferates with $M$. So in large systems one cannot only expect more transitions to occur when a parameter is varied, but also that the discontinuous jumps, which the nonparticipating occupations undergo at each transition, become smaller.

Before moving on, let us briefly discuss the case of finite particle numbers $N$, where the sharp transition becomes a crossover of finite width. This can be observed in Fig. 8. Here we plot the mean occupations versus the total particle number $N$ for a system described by the same rate matrix $R(p)$ used in Fig. 6. The five panels of Fig. 8 are obtained for parameters $p$ close to (or at) the transition labeled (I,B) in Fig. 6, with the critical parameter denoted by $p^{*}$. The first panel corresponds to a parameter well on the left-hand side of the transition. Here, asymptotically, three states become selected. When coming closer to the transition, but still staying on its left-hand side (second panel), we can observe that a preasymptotic regime appears. Namely, at large, but finite $N$ the system approaches a state with two selected states, before eventually in the asymptotic limit $N \rightarrow \infty$, a third state becomes selected as well. This third state corresponds to the triggering state $i^{<}$. The two states that appear to be selected in this preasymptotic regime correspond to those two states that are selected at the transition (middle panel). The fourth panel corresponds to a parameter, where the transition has just been passed. Here (roughly) the same preasymptotic state is found, before asymptotically for $N \rightarrow \infty$ a third state joins the group of selected states. Now the third state is given by $i^{>}$. The fifth panel is, finally, obtained for a parameter well on the right-hand side of the transition. Here again no preasymptotic regime is found. The emergence of a preasymptotic regime close to the transition implies that the fine-tuned rate matrix $R\left(p^{*}\right)$, which gives rise to two selected states, provides an accurate description of the system within a finite interval of parameters near the transition.

\section{G. Efficient algorithm for finding the selected states}

In principle, finding the unique set $\mathcal{S}$ of Bose-selected states requires to sample all possible subsets, whose number grows exponentially with $M$, until one succeeds to satisfy the conditions (80). Testing all sets by brute force quickly becomes unpractical already for moderately large values of $M$. While the mean-field occupations and especially their dependence on the total particle number can provide some guidance, this method also quickly reaches its limits when $M$ is further increased. Here we describe an efficient algorithm for finding the set of selected states. It uses the theory of transitions that we presented in the previous section.

In order to solve the problem of finding the set of selected states for a given rate-imbalance matrix $A$, we construct the auxiliary rate-imbalance matrix

$$
\tilde{A}_{i j}(p)=A_{i j}+p B_{i j}
$$

by adding the real-valued skew-symmetric matrix $B$, weighted with the real parameter $p$, to the original one. The problem defined by the new matrix $\tilde{A}(p)$ will be solved by a set $\tilde{\mathcal{S}}(p)$ of selected states. The matrix $B$ is constructed as follows: It shall possess a crosslike structure, with nonzero elements only in the column and the line labeled by $k$,

$$
B_{i j}=\delta_{i k} b_{j}-\delta_{k j} b_{i}
$$


so that

$$
\tilde{A}_{k j}(1)=A_{k j}+b_{j}>0, \quad \forall j \neq k .
$$

This condition, which corresponds to the relation (67), ensures that for $p=1$ only the state $k$ will be selected, $\tilde{\mathcal{S}}(1)=\{k\}$. Relation (89) can be achieved with minimal effort by setting

$$
b_{i}= \begin{cases}+\left|A_{k i}\right|+\varepsilon_{i}>0 & \text { if } A_{k i} \leqslant 0, \\ 0 & \text { otherwise, }\end{cases}
$$

with arbitrary $\varepsilon_{i}>0$. Our strategy will now consist of ramping the parameter $p$ down from $p=1$, where the solution $\tilde{\mathcal{S}}(1)=$ $\{k\}$ is known by construction, to $p=0$, where we would like to know the solution $\tilde{\mathcal{S}}(0)=\mathcal{S}$. During this ramp, we monitor all transitions, i.e., changes of the set $\tilde{\mathcal{S}}(p)$, that are happening, so that at the end we arrive at the desired solution. For that purpose it seems favorable (though not necessarily required) to choose the state $k$ such that a minimum of the elements $b_{j}$ defined like (90) has to be nonzero, and to choose the $\varepsilon_{i}$ different from each other, $\varepsilon_{i} \neq \varepsilon_{j}$ for $i \neq j$, in order to separate the transitions when varying $p$.

In order to follow the state of the system during the parameter ramp, we take advantage of the specific way the matrix $\tilde{A}(p)$ depends on the parameter $p$. Namely, the cross structure (88) of the matrix $B$ implies that the occupations of the system change in a linear fashion unless a transition occurs: If the vector $\tilde{v}\left(p_{0}\right)$ solves the problem $(80)$ for $\tilde{A}\left(p_{0}\right)$, then one has

$$
\tilde{\boldsymbol{v}}(p)=C(p)\left[\tilde{\boldsymbol{v}}\left(p_{0}\right)+\tilde{\boldsymbol{v}}^{\prime}\left(p_{0}\right)\left(p-p_{0}\right)\right] \text { for } p_{a}<p<p_{b},
$$

with a global normalization factor $C(p)>0$ such that $\sum_{j \in \tilde{\mathcal{S}}(p)} \tilde{\boldsymbol{v}}(p)=1$. Here the limits $p_{a}$ and $p_{b}$ are given by those values of $p$, where the set of selected state changes away from $\tilde{\mathcal{S}}\left(p_{0}\right)$ in a transition. The proof of this statement is rather technical and delegated to Appendix E, where we also describe how to obtain $\tilde{\boldsymbol{v}}^{\prime}\left(p_{0}\right)$. Expression (91) can be employed to predict the positions $p_{a}$ and $p_{b}$ of the transitions as those points, where either an element $\tilde{\nu}_{i}(p)$ of $\tilde{\boldsymbol{v}}(p)$ or an element $\tilde{\mu}_{i}(p)$ of the associated vector $\tilde{\boldsymbol{\mu}}(p)=\tilde{A}(p) \tilde{\boldsymbol{v}}(p)$ would change sign. The label $i$ of this state corresponds to the state that triggers the transition.

With these ingredients, our algorithm works as follows: Start from $p=1$, where $\tilde{\mathcal{S}}(1)=\{k\}$, and evaluate where the next transition occurs when $p$ is lowered and by which state $i^{>}$it will be triggered. Next, employ the theory of transitions described in the previous section to determine the partner state $i^{<}$, which at the transition also changes between the sets of selected and nonselected states. In this way the new set of selected states solving $\tilde{A}(p)$ after the transition has been found. Then compute where the next transition occurs when $p$ is lowered further, iterating this procedure until $p=0$ is reached. The time needed to find the set of selected states in this way scales polynomial with the system size $M$. For the random-rate model, which constitutes a rather difficult problem since on average half of the states are selected [23], we find this time to scale as $\sim M^{\alpha}$ with $\alpha \approx 4$. This allows us to find the set of selected states for systems of up to $M=1000$ states. An alternative algorithm for solving the problem (80) has recently been presented in Ref. [47] and is based on linear programming.

\section{H. Small rates and preasymptotic regime}

So far we have assumed strictly positive rates, $R_{i j}>0$, within the asymptotic theory. This assumption is reasonable in the sense that exactly vanishing rates, $R_{i j}=0$, can be viewed as a fine-tuned situation. However, obviously, we can encounter situations where some rates are much smaller than others, e.g.,

$$
R_{i j}= \begin{cases}O(r) & \text { for }(i, j) \in G, \\ O(\varepsilon r) & \text { else, }\end{cases}
$$

with $G$ denoting the subset of pairs $(i, j)$ with large rates of order $r$ and $\varepsilon \ll 1$ quantifying the suppression of small rates of order $\varepsilon r$. Such rate matrices can result from a situation where some modes are coupled much more weakly to the environment than others. Having such a situation in mind, in the following discussion we consider a rate $R_{i j}$ to be small only when also its backward rate $R_{j i}$ is small too, so that also the corresponding rate asymmetry $\left|A_{i j}\right|$ is small. ${ }^{6}$

Having some rates much smaller than others, it appears reasonable to neglect the small rates in an approximation,

$$
R_{i j} \approx R_{i j}^{a}= \begin{cases}R_{i j} & \text { for }(i, j) \in G, \\ 0 & \text { else. }\end{cases}
$$

As we argue below, such an approximation will describe the system accurately, provided that the total particle number $N$ remains below a threshold $N_{\text {thr }}$ associated with the approximation. Thus, when increasing the particle number $N$, one might encounter the following scenario: First a preasymptotic state is approached, where the occupations are well described by the asymptotic theory based on the approximate rate matrix $R_{i j}^{a}$, before eventually the true asymptotic state of the full rate matrix $R$ is reached above the threshold. This scenario can be observed in Fig. 9, where we plot the occupations of a minimal three-state model versus $N$. In this model the rates are given by

$$
\begin{gathered}
R=r\left(\begin{array}{ccc}
0 & 1 & 2 \varepsilon \\
2 & 0 & 2 \\
1 \varepsilon & 4 & 0
\end{array}\right), \quad \varepsilon=10^{-3}, \\
R^{a}=r\left(\begin{array}{lll}
0 & 1 & 0 \\
2 & 0 & 2 \\
0 & 4 & 0
\end{array}\right) .
\end{gathered}
$$

This behavior resembles the preasymptotic behavior found near transitions that we discussed at the end of Sec. IV F. In both cases the preasymptotic state is described by a fine-tuned rate matrix, either characterized by the critical parameter or by setting several matrix element to zero. However, since setting several matrix elements to zero corresponds to the fine tuning of several parameters, the set of selected states of $R^{a}$ can be quite different from that of $R$.

The appearance of a preasymptotic regime described by the approximate rate matrix (95) at intermediate particle numbers

\footnotetext{
${ }^{6}$ There can also be small rates without small backward rates, e.g., between states with a large energy separation. Not considering those rates as small in the analysis below (i.e., not exploiting the fact that they are small) does not spoil its validity.
} 


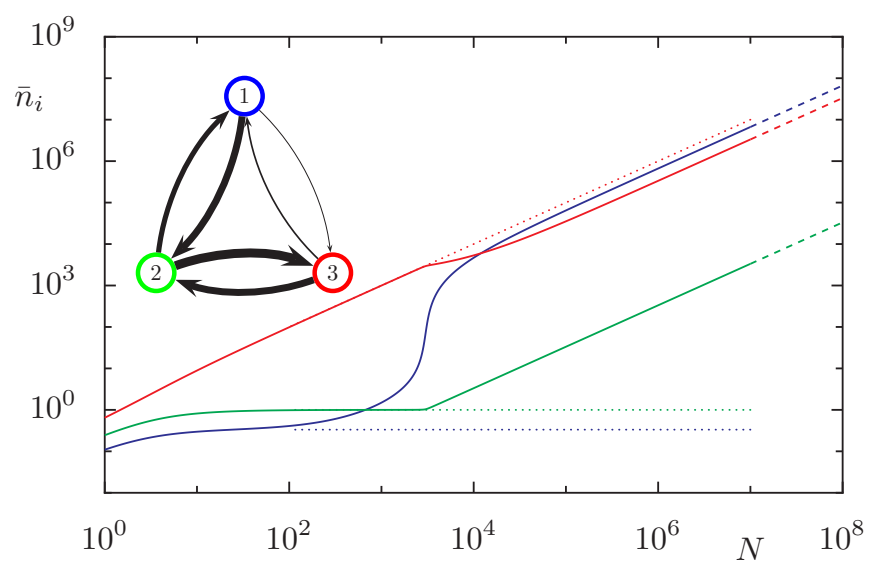

FIG. 9. (Color online) Effect of small rates for a minimal threestate model with rate matrix (94). Occupations $\bar{n}_{i}$ versus total particle number $N$ obtained using mean-field theory (solid lines) and asymptotic theory (dashed lines) for the rate matrix $R$ given by Eq. (94), which is visualized in the inset (line widths reflect rates). Furthermore, the dotted lines show occupations $\bar{n}_{i}$ obtained by the asymptotic theory for the approximate rate matrix $R^{a}$ given by Eq. (95), where the small rates have been neglected. Blue, green, and red lines describe $\bar{n}_{1}, \bar{n}_{2}$, and $\bar{n}_{3}$, respectively. Near $N \sim 10$ the system approaches a preasymptotic state with a single selected state, described by $R^{a}$, before above $N \sim 10^{3}$ the true asymptotic state is reached, where all states are selected.

$N$, as it is visible in Fig. 9 roughly for $10<N<10^{3}$, can be explained as follows. When applying the asymptotic theory, Sec. IV C, to the approximate rate matrix, where small rates are neglected, we find that the selected state 3 acquires an occupation $\sim N$, while the occupations of the nonselected states are $\sim 1$. Generally, the fact that the selected state(s) possesses an occupation much larger than the nonselected states justifies the $1 / N$ expansion (68), which underlies the asymptotic theory. This explains why the preasymptotic regime is reached near $N \sim 10$, when $N \gg 1$. However, as soon as the factor $N$ between the occupations of the selected and the nonselected states becomes comparable to the inverse suppression factor $\varepsilon^{-1} \sim 10^{3}$, the weak rates start to spoil the hierarchy of the $1 / N$ expansion based on the selected state of $R^{a}$. Namely, the product of a small rate with the occupation of a selected state $\sim r \varepsilon N$, which was neglected so far, can become comparable to the product of a large rate with the occupation of a nonselected state $\sim r$, which has been taken into account. This explains why for $N>N_{\mathrm{thr}} \sim \varepsilon^{-1}=10^{3}$ the system starts to deviate from the solution of the approximate rate matrix (describing the preasymptotic state) to approach the true asymptotic state determined by the full rate matrix.

Note that allowing for zero rates, i.e., rate matrices that are not fully connected like $R^{a}$, can have several consequences for the asymptotic theory. These are discussed in the following section.

\section{Zero rates: Not fully connected rate matrices}

So far we have assumed fully connected rate matrices within our asymptotic theory. What happens if we allow some rates to become zero? This question emerges, e.g., when computing the asymptotic state of an approximate rate matrix $R^{a}$ [Eq. (93)]. First of all, in case the rate matrix is disconnected, so that it is not possible anymore to reach every state $i$ from every other state $j$ in a sequence of quantum jumps (and vice versa), then the steady state of the system is not unique anymore [57] and will depend on the initial conditions. ${ }^{7}$ We exclude this scenario from the following discussion and focus on situations where the rate matrix is solved by a unique steady state.

In order to discuss the impact of zero rates, let us briefly recapitulate the situation where all states are coupled to all other states. In this case the coefficients $v_{i}$ and $v_{i}^{(r)}$ of the $1 / N$ expansion (68) are obtained as follows. First the leading coefficients $v_{i}$, and with that the set $\mathcal{S}$ of selected states, have to be determined by solving the problem (80). Then the subleading coefficients $v_{i}^{(r)}$ can be obtained iteratively from the hierarchy of equations that results from Eq. (70) by requiring the terms of each power of $N$ to vanish separately. If we denote the terms $\propto N^{-r}$ on the right-hand side of Eq. (70) by $I_{i}^{(r)}$, then this hierarchy of equations reads

$$
I_{i}^{(r)}\left(\boldsymbol{v}, \boldsymbol{v}^{(1)}, \ldots, \boldsymbol{v}^{(r)}\right)=0,
$$

for all $i$ and for $r=0,1,2, \ldots$ and with $\boldsymbol{v}^{(r)}$ denoting the vector of coefficients $v_{i}^{(r)}$. Now the $v_{i}^{(1)}$ are obtained by solving the set of linear equations $I_{i}^{(1)}\left(\boldsymbol{v}, v^{(1)}\right)$, with the already determined $v_{i}$ treated as parameters. Then the $v_{i}^{(2)}$ are obtained from the set of linear equations $I_{i}^{(2)}\left(\boldsymbol{v}, v^{(1)}, v^{(2)}\right)=0$, with the already determined coefficients $\nu_{i}$ and $v_{i}^{(1)}$ entering as parameters, and so on.

This procedure has to be modified for non-fully-connected rate matrices. In the following discussion we assume that $R_{i j}=0$ implies $R_{j i}=0$ and, thus, also $A_{i j}=0$, this is analogous to our assumption about the occurrence of small rates in the previous section. Let us start with the zeroth-order equation, $I_{i}^{(0)}=v_{i} \sum_{j} A_{i j} v_{j}=0$. As before, we conclude that the leading coefficients $v_{i}$ are nonzero only for a group of selected states $i \in \mathcal{S}$,

$$
\begin{gathered}
\sum_{j \in \mathcal{S}} A_{i j} v_{j}=0, \quad i \in \mathcal{S}, \\
\nu_{i}=0, \quad i \notin \mathcal{S},
\end{gathered}
$$

The set $\mathcal{S}$ of selected states has still to be determined from the requirement that the asymptotic occupations of both the selected and the nonselected states are positive. It can consist of $K$ uncoupled subsets $\mathcal{S}_{\alpha}$,

$$
\mathcal{S}=\mathcal{S}_{1} \cup \mathcal{S}_{2} \cup \cdots \cup \mathcal{S}_{K},
$$

with

$$
R_{i j}=0 \quad \text { for } \quad i \in \mathcal{S}_{\alpha}, j \in \mathcal{S}_{\beta}, \alpha \neq \beta,
$$

\footnotetext{
${ }^{7}$ If, by taking into account neglected rates of order $\varepsilon r$, the matrix is connected again, then for times longer than $1 /(\varepsilon r)$ the nonunique steady states associated with $R^{a}$ will eventually relax to the unique steady state of the full rate matrix.
} 
such that each subset $\mathcal{S}_{\alpha}$ fulfills Eqs. (97) individually,

$$
\sum_{j \in \mathcal{S}_{\alpha}} A_{i j} v_{j}=0, \quad \forall i \in \mathcal{S}_{\alpha}
$$

Without fine tuning, a solution of $\sum_{j \in \mathcal{S}} A_{i j} v_{j}=0$ is guaranteed as long as the number of states in each of the subsets $\mathcal{S}_{\alpha}$ is odd. However, the total number of selected states $M_{S}$ can now also be even. It is even (odd) if the number $K$ of uncoupled subsets $\mathcal{S}_{\alpha}$ is even (odd). In the case of fully connected rate matrices, the coefficients $v_{i}$ were determined uniquely by the set $\mathcal{S}$, Eqs. (97) and (98), as well as by the normalization condition (69). For $K>1$ this is not the case anymore. Here the relative occupation of a subset $\mathcal{S}_{\alpha}$, defined by $\nu_{\mathcal{S}_{\alpha}}=\sum_{i \in \mathcal{S}_{\alpha}} v_{i}$, is not fixed, since $v_{\mathcal{S}_{\alpha}} / v_{\mathcal{S}_{\beta}}$ with $\alpha \neq \beta$ is not determined by Eq. (97). Thus, one has $K-1$ parameters $v_{\mathcal{S}_{\alpha}}$ that yet have to be determined from the equations of higher order.

In order to investigate the first-order equations $I_{i}^{(1)}=0$ [see Eq. (70)], it is useful to define two groups of nonselected states,

$$
\overline{\mathcal{S}}=\overline{\mathcal{S}}^{\prime} \cup \overline{\mathcal{S}}^{\prime \prime},
$$

such that states that are directly coupled to selected states via nonzero rates form the set $\overline{\mathcal{S}}^{\prime}$ and states that are not coupled directly to any selected state form the set $\overline{\mathcal{S}}^{\prime \prime}$. For $i \in \overline{\mathcal{S}}^{\prime}$ they lead to the familiar result

$$
v_{i}^{(1)}=-\frac{\sum_{j \in \mathcal{S}} R_{i j} v_{j}}{\sum_{j \in \mathcal{S}} A_{i j} v_{j}}, \quad i \in \overline{\mathcal{S}}^{\prime} .
$$

Note that for states $i \in \overline{\mathcal{S}}^{\prime}$ that are coupled to selected states belonging to two subsets $\mathcal{S}_{\alpha}$ and $\mathcal{S}_{\beta}$ (or more), the right-hand side of Eq. (103) depends on the ratio $v_{\mathcal{S}_{\alpha}} / v_{\mathcal{S}_{\beta}}$, which is not determined yet. In that case the ratio $\nu_{\mathcal{S}_{\alpha}} / \nu_{\mathcal{S}_{\beta}}$ can be obtained from Eqs. (105) below. The coefficients $v_{i}^{(1)}$ with $i \in \overline{\mathcal{S}}^{\prime \prime}$ drop out of the first-order equations $\left(I_{i}^{(1)}=0\right.$ is fulfilled trivially) and must be determined from the second-order Eqs. (107) below. The first-order equations for the selected states $i \in \mathcal{S}_{\alpha}$ of a subset $\mathcal{S}_{\alpha}$ simplify [with Eq. (101)] to

$$
\begin{aligned}
0= & \sum_{j \in \mathcal{S}_{\alpha}}\left[R_{i j} v_{j}-R_{j i} v_{i}+A_{i j} v_{i} v_{j}^{(1)}\right] \\
& +\sum_{j \in \overline{\mathcal{S}}^{\prime}}\left[-R_{j i} v_{i}+A_{i j} v_{i} v_{j}^{(1)}\right], \quad i \in \mathcal{S}_{\alpha} .
\end{aligned}
$$

These equations determine the coefficients $v_{i}^{(1)}$ of the selected states $i$.

Further information can be obtained by summing Eqs. (104) over all states $i \in \mathcal{S}_{\alpha}$. This gives $0=\sum_{j \in \overline{\mathcal{S}}^{\prime}} \sum_{i \in \mathcal{S}_{\alpha}} v_{i}\left(R_{j i}+\right.$ $\left.A_{j i} v_{j}^{(1)}\right)$. Here all nonselected states $j \in \overline{\mathcal{S}}^{\prime}$ that couple only to selected states of the subset $\mathcal{S}_{\alpha}$ do not contribute to the sum, since according to Eq. (103) their occupations are given by $v_{j}^{(1)}=-\left(\sum_{i \in \mathcal{S}_{\alpha}} R_{j i} v_{i}\right) /\left(\sum_{i \in \mathcal{S}_{\alpha}} A_{j i} v_{i}\right)$. Thus, we obtain

$$
0=\sum_{j \in \overline{\mathcal{S}}_{\alpha+}} \sum_{i \in \mathcal{S}_{\alpha}} v_{i}\left(R_{j i}+A_{j i} v_{j}^{(1)}\right), \quad \forall \alpha,
$$

where $\overline{\mathcal{S}}_{\alpha+}$ denotes the set of nonselected states that couple to the subset $\mathcal{S}_{\alpha}$ and at least to one more selected state of a different subset $\mathcal{S}_{\beta}$ with $\beta \neq \alpha$. If this set $\overline{\mathcal{S}}_{\alpha+}$ is not empty, Eq. (105) can be used to determine missing relative occupations $v_{\mathcal{S}_{\alpha}} / \nu_{\mathcal{S}_{\beta}}$. We argue below that, in fact, all subsets of selected states must form a connected cluster, where two subsets $\mathcal{S}_{\alpha}$ and $\mathcal{S}_{\beta}$ are defined to be connected if they are coupled directly (via a single quantum jump of nonzero rate) to the same nonselected state(s). This guarantees that all relative occupations $v_{\mathcal{S}_{\alpha}} / \nu_{\mathcal{S}_{\beta}}$ can be determined from Eqs. (105) and (103), so that the $v_{i}$ can be determined completely.

From the second-order equations $I_{i}^{(2)}=0$, we obtain

$$
\begin{aligned}
0= & \sum_{j}\left[R_{i j} v_{j}^{(1)}-R_{j i} v_{i}^{(1)}\right. \\
& \left.+A_{i j}\left(v_{i}^{(2)} v_{j}+v_{i}^{(1)} v_{j}^{(1)}+v_{i} v_{j}^{(2)}\right)\right], \quad \forall i .
\end{aligned}
$$

These equations determine all the coefficients $v_{i}^{(1)}$ that have not been obtained yet, since all $v_{i}^{(1)}$ are coupled to each other (at least indirectly). For the missing coefficients $v_{i}^{(1)}$ of states $i \in \overline{\mathcal{S}}^{\prime \prime}$ they simplify further to

$$
0=\sum_{j \in \overline{\mathcal{S}}}\left(R_{i j} v_{j}^{(1)}-R_{j i} v_{i}^{(1)}+A_{i j} v_{i}^{(1)} v_{j}^{(1)}\right), \quad \forall i \in \overline{\mathcal{S}}^{\prime \prime},
$$

since the states $i \in \bar{S}^{\prime \prime}$ couple to nonselected states only. In these equations the coefficients $v_{j}^{(1)}$ for the states $j \in \overline{\mathcal{S}}^{\prime}$ are determined already by Eqs. (103).

The statement that all subsets of selected states must form a single connected cluster (in the sense described above) can now be shown by noting that the assumption of several mutually unconnected clusters $A, B, C, \ldots$ leads to a contradiction. Let us denote the set of nonselected states directly coupled to the selected states of cluster $X$ by $\overline{\mathcal{S}}_{X}^{\prime}$ and note that the mean particle current from one subset of nonselected states $\overline{\mathcal{S}}_{1}$ to another one $\overline{\mathcal{S}}_{2}$ is in leading order given by $J_{\overline{\mathcal{S}}_{2} \overline{\mathcal{S}}_{1}}=$ $\sum_{i \in \overline{\mathcal{S}}_{2}} \sum_{j \in \overline{\mathcal{S}}_{1}}\left(A_{i j} v_{i}^{(1)} v_{j}^{(1)}+R_{i j} v_{j}^{(1)}-R_{j i} v_{i}^{(1)}\right)$. The total current into $\overline{\mathcal{S}}^{\prime \prime}$ then reads $J_{\overline{\mathcal{S}}^{\prime \prime}}=J_{\overline{\mathcal{S}}^{\prime \prime} \overline{\mathcal{S}}^{\prime}}=J_{\overline{\mathcal{S}}^{\prime \prime}} \overline{\mathcal{S}}_{A}^{\prime}+J_{\overline{\mathcal{S}}^{\prime \prime} \overline{\mathcal{S}}_{B}^{\prime}}+\cdots$. It is directly given by summing the right-hand sides of Eqs. (107). Consequently, it vanishes in the steady state as it should, $J_{\overline{\mathcal{S}}^{\prime \prime}}=0$. The total current into cluster $A$ reads $J_{\overline{\mathcal{S}}_{A}^{\prime}}=J_{\overline{\mathcal{S}}_{A}^{\prime} \overline{\mathcal{S}}^{\prime \prime}}+J_{\overline{\mathcal{S}}_{A}^{\prime} \overline{\mathcal{S}}_{B}^{\prime}}+J_{\overline{\mathcal{S}}_{A}^{\prime} \overline{\mathcal{S}}_{C}^{\prime}}+\cdots$. Obviously, it should also vanish in the steady state. However, generically this is is not possible for more than a single cluster. Namely (without fine tuning), the individual terms $J_{\overline{\mathcal{S}}_{A}^{\prime} \overline{\mathcal{S}}^{\prime \prime}}, J_{\overline{\mathcal{S}}_{A}^{\prime} \overline{\mathcal{S}}_{B}^{\prime}}, \ldots$, containing the coefficients $v_{i}^{(1)}$ determined from Eqs. (103) and (107), can neither be expected to vanish individually nor to cancel each other. In contrast, for a single cluster, one has $J_{\overline{\mathcal{S}}_{A}^{\prime}}=J_{\overline{\mathcal{S}}_{A}^{\prime} \overline{\mathcal{S}}^{\prime \prime}}=$ $-J_{\overline{\mathcal{S}}^{\prime \prime}}=0$, as required.

From the rather technical discussion of the preceding paragraphs, we can now draw several important conclusions. First of all, Eqs. (97) and (98) imply that Bose selection is still predicted to occur, i.e., only a subset $\mathcal{S}$ of the single-particle states has occupations that grow with the total particle number

$$
\bar{n}_{i}=v_{i} N
$$

Second, the asymptotic occupations of the nonselected states are still determined by the first-order coefficient $v_{i}^{(1)}$, so that their occupations saturate for large $N$. (In contrast, if $v_{i}^{(2)}$ would describe the leading contribution to the occupations of a state $i$, it would become unpopulated in the limit of large particle 
numbers). This is true also for states contained in $\overline{\mathcal{S}}^{\prime \prime}$ that are not directly coupled to a selected state. Both conclusions, Bose selection and saturation, are confirmed by the preasymptotic state that can be observed in Fig. 9 for $10^{1} \lesssim N \lesssim 10^{3}$, which is approximately given by the asymptotic state of the rate matrix $R^{a}$ [Eq. (95)].

Finally, a third conclusion is that for rate matrices that are not fully connected the set of selected states $\mathcal{S}$ is not determined by the conditions (80) anymore. Namely, a negative $\mu_{i}$ guarantees a positive asymptotic occupation $v_{1}^{(1)}$ of a nonselected state $i \in \overline{\mathcal{S}}^{\prime}$, but not for a nonselected state $i \in \overline{\mathcal{S}}^{\prime \prime}$. This implies that we cannot apply the efficient algorithm presented in Sec. IV G in order to find the set of selected states [neither can the algorithm of Ref. [47] be used, which is also based on the conditions (80)]. It seems likely that the set of selected states of the mean-field equations is still unique and determined by the requirement of having positive occupations, as the full many-body master equation possesses a unique steady state. However, unlike in the case of fully connected rate matrices, we have no proof for this statement.

Let us illustrate the above reasoning using the minimal example given by the rate matrix $R^{a}$ defined in Eq. (95) of the previous section. The corresponding rate-asymmetry matrix reads

$$
A^{a}=r\left(\begin{array}{rrr}
0 & -1 & 0 \\
1 & 0 & -2 \\
0 & 2 & 0
\end{array}\right)
$$

Thus, if we were allowed to solve the problem (80) to find the set of selected states and the asymptotic occupations, we would find two disconnected clusters of selected states given by $\mathcal{S}_{1}=\{1\}$ and $\mathcal{S}_{2}=\{3\}$. Namely,

$$
\boldsymbol{\mu}=r\left(\begin{array}{rrr}
0 & -1 & 0 \\
1 & 0 & -2 \\
0 & 2 & 0
\end{array}\right)\left(\begin{array}{c}
v_{1} \\
0 \\
v_{3}
\end{array}\right)=\left(\begin{array}{c}
0 \\
r\left(v_{1}-2 v_{3}\right) \\
0
\end{array}\right)
$$

solves problem (80) nonuniquely for $0<v_{1}<2 / 3$ and $v_{3}=$ $1-v_{1}$. However, this is not the true solution. Namely, Eq. (105) for $\alpha=1$ simplifies to $0=v_{1}\left(R_{21}+A_{21} v_{2}^{(1)}\right)=$ $v_{1}\left(2+v_{2}^{(1)}\right)$ from which $v_{1}=0$ follows in contradiction to Eqs. (80). This demonstrates that Eq. (80) cannot be used in order to determine the selected states in the case of non-fully-connected rate matrices.

From Fig. 9, where $R^{a}$ describes the preasymptotic regime $\left(10^{1} \lesssim N \lesssim 10^{3}\right)$, one can infer that only state 3 will be selected. Let us, therefore, solve Eqs. (97), (98), (103), and (106) for the ansatz

$$
\mathcal{S}=\{3\}
$$

The zeroth-order equations (97) and (98) are solved trivially by

$$
v_{3}=1, \quad v_{1}=v_{2}=0 .
$$

Then $v_{2}^{(1)}$ is obtained from Eq. (103) and reads

$$
v_{2}^{(1)}=-\frac{R_{23}^{a}}{A_{23}^{a}}=1,
$$

while Eq. (105) is trivially fulfilled since $\overline{\mathcal{S}}_{\alpha+}$ is empty. Finally, $v_{1}^{(1)}$ results from Eq. (106) for $i=1$,

$$
v_{1}^{(1)}=\frac{R_{12}^{a} v_{2}^{(1)}}{R_{21}^{a}-A_{12}^{a} v_{2}^{(1)}}=\frac{1}{3},
$$

We can see that the initial assumption $\mathcal{S}=\{3\}$ is confirmed by the fact that we obtained meaningful positive occupation numbers. The just-obtained asymptotic occupations for the rate matrix $R^{a}$ are plotted as dotted lines in Fig. 9 and provide a good description of the preasymptotic state.

For completeness, we finally present a simple example for a situation where the set of selected states consists of two uncoupled subsets. It is given by a model of four states with rate matrix

$$
R=r\left(\begin{array}{llll}
0 & 2 & 0 & 1 \\
1 & 0 & 3 & 0 \\
0 & 1 & 0 & 4 \\
5 & 0 & 1 & 0
\end{array}\right)
$$

The occupations plotted in Fig. 10 show that the set of selected states contains the two uncoupled states 2 and 4 ,

$$
\mathcal{S}=\mathcal{S}_{1} \cup \mathcal{S}_{2}, \quad \text { with } \quad \mathcal{S}_{1}=\{2\} \text { and } \mathcal{S}_{2}=\{4\} .
$$

The case of zero rates has recently also been discussed by Knebel et al. for the Lotka-Volterra equations of motion $[46,47]$,

$$
\dot{\bar{n}}_{i}=\bar{n}_{i} \sum_{j} A_{i j} \bar{n}_{j}
$$

These equations correspond to the leading-order high-density approximation (59) of the mean-field equation (31), with $\sigma=1$ for bosons. These leading-order equations describe the dynamics of the Bose gas on an intermediate time scale, before eventually the subleading terms of Eq. (31), which are linear in the occupations, become relevant and determine the steady state. Knebel et al. show that under the evolution described by Eq. (117) the occupations of some states $i$ die out exponentially fast, while the other

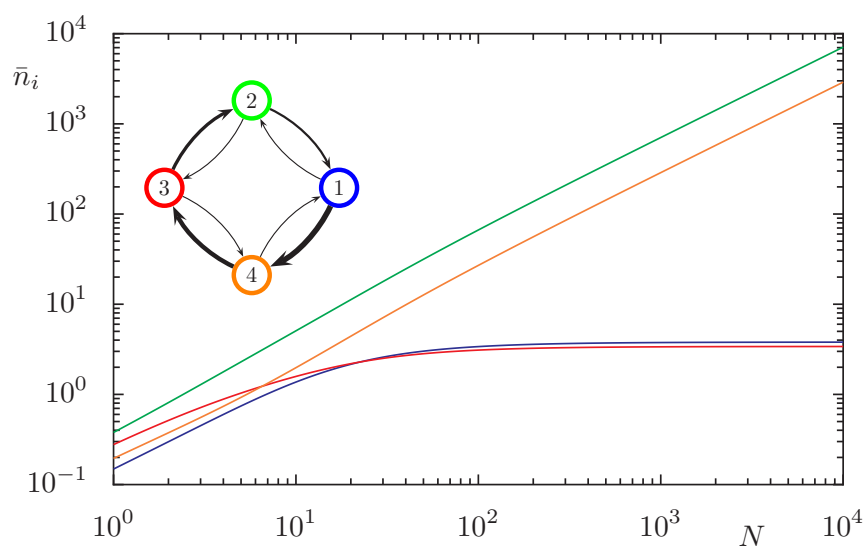

FIG. 10. (Color online) Example for Bose selection of two uncoupled states. Mean occupations obtained from mean-field theory ( $\bar{n}_{1}$ blue, $\bar{n}_{2}$ green, $\bar{n}_{3}$ red, $\bar{n}_{4}$ orange) vs the total particle number $N$ for the rate matrix (115), which is visualized in the inset (line widths reflect rates). The two selected states 2 and 4 are not coupled directly. 


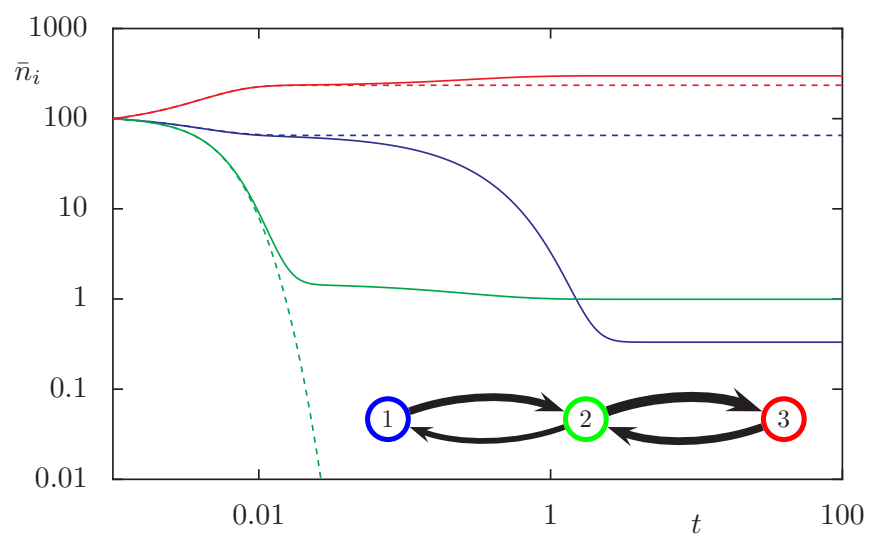

FIG. 11. (Color online) Comparison between the dynamics of the mean-field equations (31) (solid lines) and the Lotka-Volterra equations (117) (dashed lines) for the rate matrix (95), which is visualized in the inset (line widths reflect rates), for $N=300$ particles initially uniformly distributed. While in both cases the population in state 2 (green) decays on an intermediate time scale, the population in state 1 (blue) decays on a longer time scale in the mean-field equations only leading to a single condensate in state 3 (red).

states retain nonzero occupations. Interestingly, those states retaining nonzero occupations are determined by the very same condition (80) that we found to determine the selected states for fully connected rate matrices. That means in the case of fully connected rate matrices the selected states are determined already by the leading-order equation (117). Note the general difference between the mean-field equation on the one hand and the Lotka-Volterra equation on the other. While in the first case the nonselected states retain a small but nonzero occupation, they die out completely in the latter case.

In the case of non-fully-connected rate matrices Eqs. (80) still determine uniquely which occupations die out under the dynamics of Eq. (117) [47]. Thus, the conditions (80) still describe a dynamical selection mechanism happening on an intermediate time scale. However, in order to compute the (true) steady state approached in the long-time limit, also higher-order equations [Eqs. (103), (104), and (106)] have to be taken into account. As a result, the set of selected states in the steady state can be different from that obtained from conditions (80).

Let us illustrate the above reasoning using the example of the not fully connected rate matrix (95). Figure 11 shows the different dynamics of this system for both the full mean-field equations (31) and the Lotka-Volterra equations of motion (59). In the limit of large $N$ the population of state 2 decays on the intermediate time scale, because the conditions (80) predict an extinction of occupations $n_{2}$ on the level of the Lotka-Volterra equations [see Eq. (110)]. Eventually, however, when higher-order terms become relevant in the full mean-field equations of motion, also the occupation of state 1 decays so that only state 3 is selected as predicted in Eq. (111). This is contrasted by the Lotka-Volterra system, which remains in the situation with two condensates in the states 1 and 3 .

\section{J. Asymptotic theory beyond mean field}

Our theoretical description of Bose selection has so far been based on mean-field theory. The data presented in Fig. 4 for a tight-binding chain in and out of equilibrium suggests that mean-field theory provides a rather good approximation to the mean occupations. Namely, deviations between the mean-field results (thick solid lines) and the exact Monte Carlo data (crosses) are visible only for nonselected states. Where visible deviations occur, they are still rather small and captured by the augmented mean-field theory (thin solid lines) introduced in Sec. III C. Such good agreement can generally not be expected for the number fluctuations of macroscopically occupied selected modes, since mean-field theory does not comply with the conservation of the total particle number.

In this section we investigate corrections to mean-field theory in the asymptotic limit of large total particle number $N$, as they are described by the augmented mean-field theory. For simplicity, we consider the case of fully connected rate matrices. We explain why mean-field theory accurately describes the occupations of the selected states and that their correlations, such as number fluctuations, deviate from mean-field theory in a universal fashion. Moreover, we argue that the set of selected states is well described by a Gaussian state projected to the space of sharp particle number $N$.

Within the augmented mean-field theory (Sec. III C) the state of the system is described not only by the mean occupations $\bar{n}_{i}=\left\langle\hat{n}_{i}\right\rangle$, like in mean-field theory, but also in terms of the nontrivial two-particle correlations $\zeta_{i j}=\left\langle\hat{n}_{i} \hat{n}_{j}\right\rangle-$ $\left\langle\hat{n}_{i}\right\rangle\left\langle\hat{n}_{j}\right\rangle$. In order to derive an augmented mean-field theory for the asymptotic limit of large total particle numbers $N$, we do not only expand the mean occupations with respect to the inverse particle number, but at the same time also the nontrivial two-particle correlations,

$$
\begin{gathered}
\bar{n}_{i}=N v_{i}+v_{i}^{(1)}+N^{-1} v_{i}^{(2)}+N^{-2} v_{i}^{(3)}+\cdots, \\
\zeta_{k i}=N^{2} \xi_{k i}+N \xi_{k i}^{(1)}+\xi_{k i}^{(2)}+N^{-1} \xi_{k i}^{(3)} \cdots .
\end{gathered}
$$

Moreover, we choose again the normalization conditions

$$
\sum_{i} v_{i}=1, \quad \sum_{i} v_{i}^{(r)}=0,
$$

which fix the total particle number $N=\sum_{i} \bar{n}_{i}$ in leading order, as well as the conditions

$$
\sum_{i j} \xi_{i j}=0, \quad \sum_{i j} \xi_{i j}^{(r)}=0,
$$

ensuring that the fluctuations of the total particle number $\Delta N=\sum_{i j} \zeta_{i j}$ vanish.

We now insert the expansions (118) and (119) into the augmented mean-field equations (45) and (46), with $\sigma=1$ for bosons and with the left-hand side set to zero in order to obtain the steady state. In the resulting equations we ask that all terms belonging to a certain power of $N$ vanish independently. In this way, we obtain the set of coupled nonlinear equations,

$$
\begin{gathered}
0=\sum_{j} A_{i j}\left[v_{i} v_{j}+\xi_{i j}\right], \\
0=\sum_{j}\left[A_{k j} v_{k} \xi_{i j}+A_{i j} v_{i} \xi_{k j}+\left(A_{k j}+A_{i j}\right) v_{j} \xi_{k i}\right],
\end{gathered}
$$

for the leading order. 
Remarkably, we can solve these equations by making the simple ansatz

$$
\xi_{k i}=x\left(\delta_{k i} v_{k}-v_{k} v_{i}\right)
$$

for the leading nontrivial correlations $\xi_{i j}$, with $x$ being a free parameter. The relative weight of both terms in the bracket is chosen such that the condition (121) is obeyed. Entering the ansatz (124) into Eqs. (122) and (123) reduces these equations to the much simpler conditions

$$
v_{i} \sum_{j} A_{i j} v_{j}=0 .
$$

These equations are identical to the leading-order conditions (71) of the asymptotic mean-field theory. Using the same arguments as in the conventional asymptotic mean-field theory, we have to conclude that the solution must be of the form

$$
\begin{gathered}
\sum_{j \in \mathcal{S}} A_{i j} v_{j}=0, \quad i \in \mathcal{S}, \\
v_{i}=0, \quad i \notin \mathcal{S} .
\end{gathered}
$$

Equations (126) and (127) imply Bose selection. Only a subset $\mathcal{S}$ of selected states have nonvanishing occupations in leading order. The set $\mathcal{S}$ has to be determined by the requirement to have positive occupations both for selected and nonselected states. The asymptotic occupations of the latter are given by $v_{i}^{(1)}$ and have to be determined in the next order. Note that the set $\mathcal{S}$ obtained within the augmented theory can be different from the one obtained within mean-field theory. Namely, the occupations of the nonselected states differ in both theories, so that in the augmented theory, e.g., a transition where $\mathcal{S}$ changes might be shifted away from the critical mean-field parameter. However, as long as the set of selected states is the same in both theories, the mean-field result for the asymptotic occupations of the selected states is not corrected anymore. This explains the excellent agreement between mean-field theory, augmented mean-field theory, and Monte Carlo results for the selected-state occupations in Fig. 4.

According to the ansatz (124), we find the asymptotic correlations among the selected states to be given by

$$
\left\langle\hat{n}_{i} \hat{n}_{j}\right\rangle=(1-x) \bar{n}_{i} \bar{n}_{j}+x \bar{n}_{i} \delta_{i j}, \quad i, j \in \mathcal{S} .
$$

This is an intriguing result. It implies that the correlations and fluctuations are determined solely by the mean occupations and a single parameter $x$. The scaled two-particle correlations for particles in different selected states,

$$
g_{i j}=\frac{\left\langle\hat{n}_{i} \hat{n}_{j}\right\rangle}{\left\langle\hat{n}_{i}\right\rangle\left\langle\hat{n}_{j}\right\rangle}=1-x, \quad i, j \in \mathcal{S}, i \neq j,
$$

asymptotically approach all the same value, which is reduced by $x$ with respect to the mean-field result.

This very same parameter $x$ also determines the asymptotic number fluctuations of the Bose-selected modes,

$$
\Delta n_{i}^{2} \equiv \zeta_{i i}=x N^{2}\left(1-v_{i}\right) v_{i}=x\left(N-\bar{n}_{i}\right) \bar{n}_{i}, i \in \mathcal{S} .
$$

This equation implies that (in leading order) the number fluctuations vanish if we have a single condensate in the state $i=k$, so that $v_{k}=1$. This is a consequence of the conservation of the total particle number that is incorporated in the augmented mean-field theory. It contrasts with the Gaussian result (35) obtained within the non-number-conserving meanfield theory, which for bosons $(\sigma=1)$ reads $\Delta n_{i}^{2}=\bar{n}_{i}+\bar{n}_{i}^{2}=$ $N^{2}\left(v_{i}+1 / N\right) v_{i}$. Note, however, that as soon as a system features several condensates (macroscopically occupied selected states), their number fluctuations (130) will typically be of the order of the total particle number. This reflects the fact that each condensate is effectively in contact with a particle reservoir given by the other ones.

The requirement $\Delta n_{i}^{2}>0$ tells us that $x$ is positive. Moreover, it is reasonable to assume that the number fluctuations will not be much larger than those obtained within the non-number-conserving mean-field theory, so that $\Delta n_{i}^{2} \lesssim \bar{n}_{i}^{2}$ for all $i \in \mathcal{S}$. Thus, an estimate for an upper bound for $x$ is determined by the selected state $i$ with the smallest occupation $\bar{n}_{i}=v_{i} N$. Therefore,

$$
0<x \lesssim \frac{v_{\min }}{1-v_{\min }}, \quad v_{\min }=\min _{i \in \mathcal{S}} v_{i}
$$

The precise value of $x$ has to be obtained, however, from the first-order equations. These equations are rather involved and we do not discuss them here. They also describe small beyond-mean-field corrections for the asymptotic occupations, correlations, and fluctuations of the nonselected states.

In Fig. 12 we compare the augmented theory (solid lines) with Monte Carlo results (crosses with error bars), ordinary mean-field theory (dotted lines), and the asymptotic prediction (128) for the selected states (dashed lines), using the model system of Fig. 4(c). The comparison with the Monte Carlo data shows that the augmented mean-field theory provides an excellent approximation for the mean occupations $\bar{n}_{i}$ [panel (a)], where the ordinary mean-field theory shows small deviations for the nonselected states [see Fig. 4(c)]. For the two-particle correlations $\left\langle\hat{n}_{i} \hat{n}_{j}\right\rangle$ shown in Figs. 12(b)-12(f), the augmented mean-field theory still provides a rather good description, though small systematic deviations with respect to the exact Monte Carlo results are now visible, while mean-field theory is not reliable anymore.

The relative number fluctuations $\Delta n_{i}^{2} / \bar{n}_{i}^{2}=\zeta_{i i} / \bar{n}_{i}^{2}$ for the selected states [panel (c)] show strong deviations from meanfield theory, once Bose selection sets in near $N=10^{2}$ [see panel (a)] so that the selected modes acquire "extensive" occupations. This agrees with our expectation that mean-field theory is not able to describe the condensate fluctuations for a system with sharp particle number. The condensate fluctuations are found to be consistent with the asymptotic prediction (130) for $x \approx 0.018$. Note that the selected state with the smallest occupation (roughly 4\%) has asymptotic number fluctuations that are only half as large as the mean-field prediction, even though the other two condensates are large enough to serve as a reservoir. Thus, $x$ is roughly given by $v_{\min } / 2$ in agreement with the estimate (131).

The other quantities displayed in Fig. 12 are not expected to exhibit such drastic deviations of orders of magnitude from mean-field theory, as we observed them for the condensate fluctuations. Panel (e) shows the scaled correlations (129) among the selected states. The augmented theory asymptotically approaches the universal value $1-x$, with $x \approx 0.018$. Noticeable deviations of up to $30 \%$ occur before reaching the asymptotic regime, whereas the deviation from the mean-field result 1 become rather small asymptotically since $x \ll 1$. 

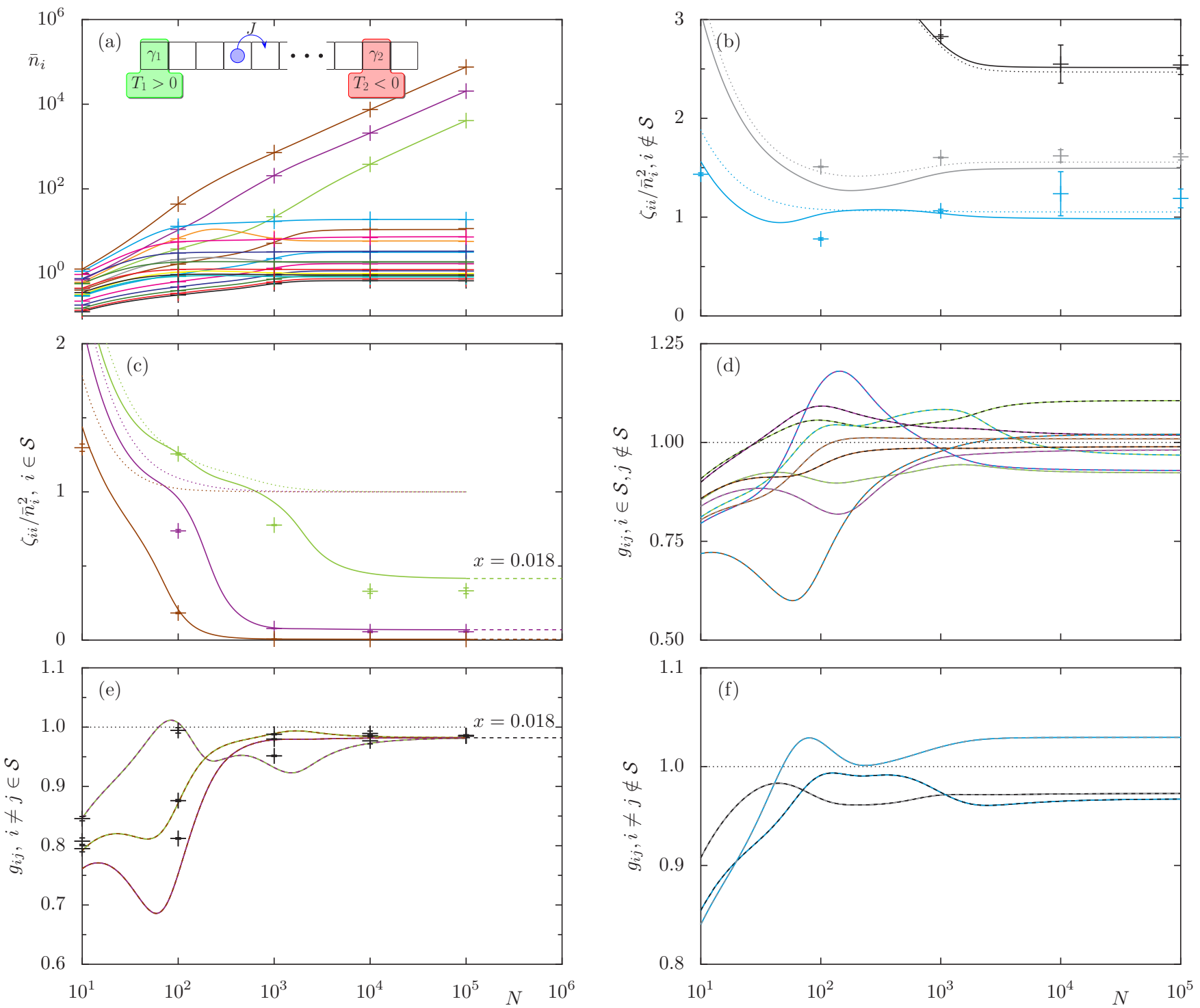

FIG. 12. (Color online) Augmented mean-field theory (solid lines) versus mean-field theory (dotted lines) and Monte Carlo simulations (crosses with error bars) for the tight-binding chain with parameters as in Fig. 4(c). All colors are consistent with panel (a) (and also Fig. 4); lines describing correlations between two states have alternating color. (a) Mean occupations [corresponding to thin solid lines in Fig. 4(c)]. (b) Relative number fluctuations $\Delta n_{i}^{2} / \bar{n}_{i}^{2}=\zeta_{i i} / \bar{n}_{i}^{2}$ for three exemplary nonselected states. (c) Relative number fluctuations $\Delta n_{i}^{2} / \bar{n}_{i}^{2}=\zeta_{i i} / \bar{n}_{i}^{2}$ of the selected states. (d) Correlations $g_{i j}$ between the selected and three exemplary nonselected states. (e) Correlations $g_{i j}$ among selected states $i \neq j$. (f) Correlations $g_{i j}$ among exemplary nonselected states $i \neq j$.

Similar behavior, i.e., larger deviations of up to a few tens of a percent for small particle numbers that are reduced slightly in the asymptotic regime, can be observed also in the remaining plots of the figure. Panel (b) displays the relative number fluctuations $\Delta n_{i}^{2} / \bar{n}_{i}^{2}$ for three exemplary nonselected states. Relative correlations $g_{i j}$ between selected states and exemplary nonselected states as well as among exemplary nonselected states are plotted in panels (d) and (f), respectively.

A deeper understanding of the findings presented so far in this section, can be gained by noting that the selected states are asymptotically described by a projected Gaussian state. This can be seen as follows. For bosons in the steady state, the full many-body rate equation (13) takes the form

$$
0=\sum_{i j}\left(1+n_{j}\right) n_{i}\left[R_{i j} p_{\boldsymbol{n}_{j i}}-R_{j i} p_{\boldsymbol{n}}\right],
$$

where $p_{\boldsymbol{n}}$ is the full occupation-number distribution. Let us accept that there is a group of selected states, whose occupations will grow with the total particle number $N$ while all other occupations saturate. Asymptotically, for $N \rightarrow \infty$, we can then neglect all nonselected states and safely approximate $\left(1+n_{j}\right) \approx n_{j}$, so that

$$
0=\sum_{i, j \in \mathcal{S}} n_{j} n_{i}\left[R_{i j} p_{\boldsymbol{n}_{j i}}-R_{j i} p_{\boldsymbol{n}}\right] .
$$

We can now show that this equation is solved by the projected Gaussian state (39). For this state one finds that $p_{\boldsymbol{n}_{j i}}=e^{\eta_{i}-\eta_{j}} p_{\boldsymbol{n}}$ for the probability of finding the system in the Fock state $\left|\boldsymbol{n}_{j i}\right\rangle$ obtained from $|\boldsymbol{n}\rangle$ by transferring one particle from $i$ to $j$. Moreover, according to Eq. (33) one has $e^{\eta_{i}}=1-1 / \bar{n}_{i} \simeq 1$. Here we have used that, asymptotically, 
the mean occupations of the projected Gaussian state become identical to that of the nonprojected Gaussian state. This implies the intuitive statement that for the projected Gaussian state, the probabilities for finding the system in the almost identical Fock states $\left|\boldsymbol{n}_{j i}\right\rangle$ and $|\boldsymbol{n}\rangle$ asymptotically become identical, $p_{\boldsymbol{n}_{j i}} \simeq p_{\boldsymbol{n}}$. Thus, plugging the projected Gaussian state into the right-hand side of Eq. (133), we obtain

$$
p_{\boldsymbol{n}} \sum_{i, j \in \mathcal{S}} n_{j} n_{i}\left[R_{i j} e^{\eta_{i}-\eta_{j}}-R_{j i}\right] \simeq p_{\boldsymbol{n}} \sum_{i, j \in \mathcal{S}} n_{j} n_{i} A_{i j}=0
$$

since $A_{i j}=-A_{j i}$. We have shown that asymptotically in the limit $N \rightarrow \infty$ the full number distribution of the selected states is given by a projected Gaussian state. An important consequence is that mean-field theory provides the exact asymptotic mean occupations of the selected states. Another consequence is that correlations $\left\langle\hat{n}_{i} \hat{n}_{j}\right\rangle$ with $i, j \in \mathcal{S}$ and, therefore, also the parameter $x$, must be determined completely by the asymptotic mean occupations $N v_{i}$ of the selected states.

\section{K. Heat flow through the system: The role of fragmented condensation and pseudotransitions}

Nonequilibrium steady states of a driven-dissipative quantum system typically feature a steady heat flow between the system and its bath(s). This heat flow is described by Eq. (11) in the case of an autonomous system and by Eq. (10) for a periodically driven system. For bosons $(\sigma=1)$ in a steady state, these equations read

$$
Q_{b}=\sum_{i j}\left(E_{i}-E_{j}\right) R_{j i}^{(b)}\left[\left\langle\hat{n}_{i}\right\rangle+\left\langle\hat{n}_{i} \hat{n}_{j}\right\rangle\right]
$$

for the heat flow from an autonomous system into bath $b$ and

$$
Q=\sum_{m} \sum_{i j}\left(\varepsilon_{i}-\varepsilon_{j}-m \hbar \omega\right) R_{j i}^{(m)}\left[\left\langle\hat{n}_{i}\right\rangle+\left\langle\hat{n}_{i} \hat{n}_{j}\right\rangle\right]
$$

for the heat flow from a Floquet system into a bath. In this section, we investigate such heat flow in the regime of Bose selection. The dominant processes contributing to the heat flow will be identified. They are found to be given by transitions between different selected states and, for the Floquet system, also by pseudotransitions [corresponding to terms with $i=j$ and $m \neq 0$ in Eq. (136)] associated with a selected state.

In Fig. 13 we present data obtained for a tight-binding chain that is driven between two heat baths, one of positive temperature and a population-inverted one modeled by a negative temperature. This system corresponds to the one of Fig. 4(c), but with the particle number fixed and with the relative coupling between both baths, $\gamma_{2} / \gamma_{1}$, varied. In panel (a) we plot the mean occupations versus the parameter $p=\left(1+\gamma_{1} / \gamma_{2}\right)^{-1}$, which increases with $\gamma_{2} / \gamma_{1}$. One can observe several transitions. For $p=0$, where the system is only coupled to bath $b=1$, a single state (the ground state) is selected as indicated by a large occupation. This corresponds to equilibrium Bose condensation. At a critical coupling to the second bath, near $p=0.2$, three states become selected. Increasing the coupling to the second bath further, various transitions occur, where the set of selected states changes. Eventually, roughly from $p=0.75$ on only the

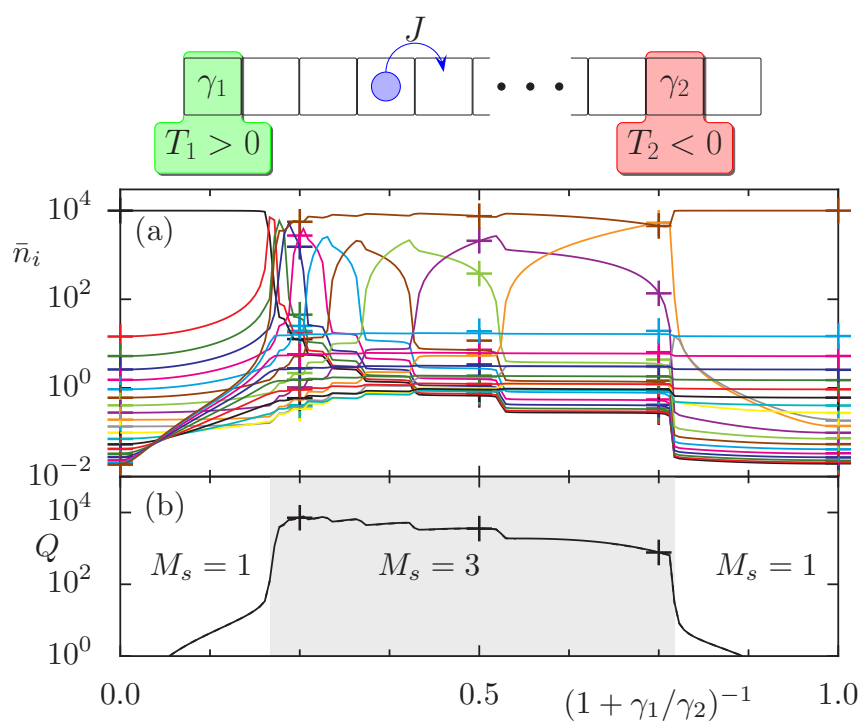

FIG. 13. (Color online) Tight-binding chain with $M=20$ sites coupled to two heat baths. Parameters as in Fig. 4(c), but for fixed $N=$ $10^{4}$ and versus relative coupling strength $\gamma_{2} / \gamma_{1}$. (a) Mean occupations obtained from mean-field theory (solid lines), augmented mean-field theory (dashed line, indistinguishable from mean-field result), and Monte Carlo simulations (crosses). Color code like in Fig. 4, on the left-hand (right-hand) side the occupation decreases (increases) with energy. (b) Heat flow through the system from the hotter negativetemperature bath into the positive-temperature bath.

most excited state will be selected, corresponding to the equilibrium situation at $p=1$, where the system is coupled to the population-inverted bath 2 only. In panel (b) we plot the heat flow from the hotter population-inverted bath through the system into the cooler positive-temperature bath versus $p$. We can clearly see that the heat flow increases dramatically (by more than two orders of magnitude), when fragmented Bose condensation with more than just one selected state occurs.

This effect, which has been reported already in Ref. [23], can be understood intuitively. Namely, in order to exchange energy with the system, the bath has to drive transitions between states $i$ and $j$ in the system. The larger the occupations of $i$ and $j$, the larger will be the rate of the corresponding transition. Therefore, the most effective way of exchanging energy with the system is to drive transitions between two largely occupied states. And this is possible only if more than just one state is selected. This effect might be employed to control the heat conductivity of a bosonic system by switching between one and three selected states.

In Fig. 14 we show results for a periodically driven tightbinding chain coupled to a heat bath. This system corresponds to the one of Fig. 4(d), but with the particle number fixed and with the dimensionless driving strength $\gamma_{\omega}$ varied. From the mean occupations plotted in panel (a), we can observe that for small $\gamma_{\omega}$ a single-particle Floquet state is selected, which is connected adiabatically to the ground state of the undriven system with $\gamma_{\omega}=0$. Roughly at $\gamma_{\omega}=0.25$ and $\gamma_{\omega}=1.5$ the selected state changes in transitions, but still only a single state is selected. Only for a driving strength of about $\gamma_{\omega}=2$, a parameter window is reached, where three states become selected and acquire large occupations. 


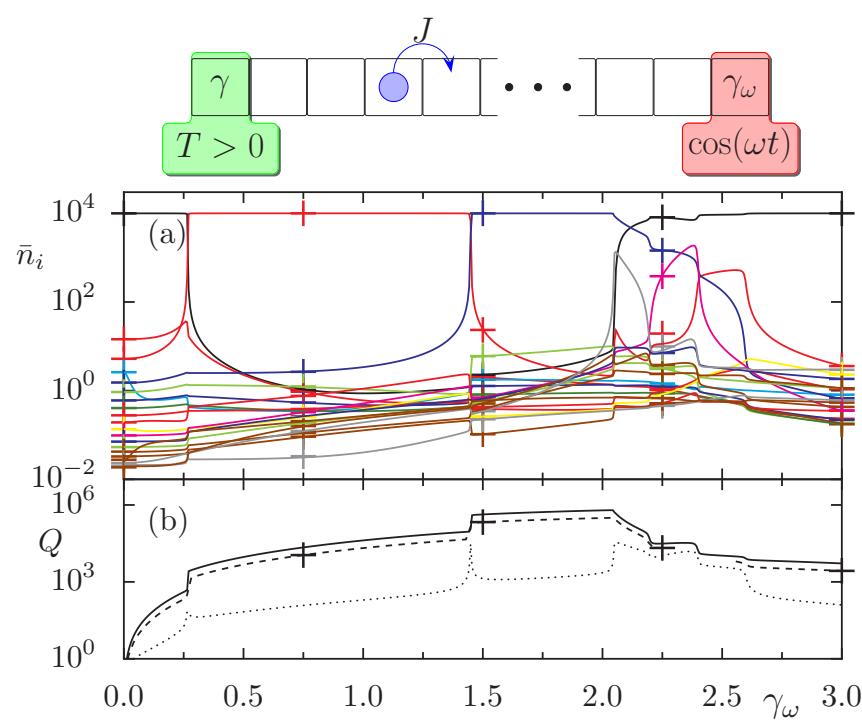

FIG. 14. (Color online) Periodically driven tight-binding chain with $M=20$ sites coupled to a heat bath. Parameters as in Fig. 4(d), but for fixed $N=10^{4}$ and versus dimensionless driving strength $\gamma_{\omega}$. (a) Mean occupations obtained from mean-field theory (solid lines) and Monte Carlo simulations (crosses). Color code like in Fig. 4; on the left-hand side the occupation decreases (increases) with energy. (b) Heat flow from the driven system into the bath obtained from mean-field theory (solid line), augmented mean-field theory (dashed line), and Monte Carlo simulations (crosses). The dotted line is the mean-field heat flow without the contribution from pseudotransitions.

The heat flow from the system into the bath is plotted in panel (b) of Fig. 14. In contrast to the autonomous chain, we can observe that the heat flow grows strongly, despite the fact that we have only a single selected state. This effect can be attributed to pseudotransitions [58] associated with rates $R_{i j}^{(m)}$ with $i=j$ and $m \neq 0$. In these processes the bath energy changes by $m \hbar \omega$, while the system's state is not altered. Thus, the bath can effectively exchange energy with the system by driving pseudotransitions for a single strongly occupied (Bose selected) Floquet mode. This interpretation is supported by the dotted line, showing the share $Q^{\prime}$ of the heat flow not related to pseudotransitions,

$$
Q^{\prime}=\sum_{m} \sum_{i, j(i \neq j)}\left(\varepsilon_{i}-\varepsilon_{j}+m \hbar \omega\right) R_{j i}^{(m)}\left[\left\langle\hat{n}_{i}\right\rangle+\left\langle\hat{n}_{i} \hat{n}_{j}\right\rangle\right] .
$$

Away from the undriven limit $\gamma_{\omega}=0$ and as long as only one Floquet mode $i$ acquires a large occupation, $Q^{\prime}$ is typically two orders of magnitude smaller than the full heat flow and, thus, negligible. That means that practically all the heat flow is based on pseudotransitions; the double sum in Eq. (136) is dominated by the terms with $i=j . Q^{\prime}$ becomes significant only when several states have a large occupation. As one can clearly observe in Fig. 14(b), this happens both near transitions, where two states are selected (see Sec. IV F), and for $2 \lesssim \gamma_{\omega} \lesssim 2.6$, where three states are selected. Here an efficient heat exchange with the bath can be achieved by driving transitions between these largely occupied states, like for the autonomous system.

In Fig. 14(b), we can also observe a noticeable difference between the heat flow obtained from mean-field theory (solid line) and augmented mean-field theory (dashed line), in contrast to the autonomous system where both theories show very good agreement [on the logarithmic scale of Fig. 14(b) both lines overlap]. This is also a consequence of the strong impact of pseudotransitions in the condensate mode, which are determined by the condensate fluctuations, a quantity that is overestimated by mean-field theory. This confirms our conclusion that, thanks to pseudotransitions not present in autonomous systems, a bosonic Floquet system can be a good heat conductor even when most of its particles form a single Bose condensate.

In conclusion, departing from equilibrium offers interesting possibilities to control the heat conductivity of a bosonic quantum system, which might be relevant for technological applications.

\section{IDEAL FERMI GASES}

In this section, we will briefly demonstrate that the theory of Sec. II and the methods presented in Sec. III can also be employed to describe the properties of ideal Fermi gases. As a motivation, we note that the physics of such drivendissipative Fermi gases will have to play an important role, for example, for the realization of Floquet topological insulators. These systems are based on lattice potentials that are forced periodically in time such that they possess a topologically nontrivial quasienergy band structure giving rise to a quantized (spin) Hall conductivity, when one band is filled completely. Proposals for Floquet topological insulators consider irradiated electronic systems like graphene [7] and semiconductor heterostructures [53]; conceptually different schemes for the Floquet engineering of topological band structures have been, moreover, proposed in the context of ultracold atomic quantum gases in optical lattices [71,72]. First experimental evidence of a (quantized) Hall conductivity in such systems has been observed with ultracold atoms in optical lattices [10,11]. These systems are well isolated from their environment. However, achieving this goal in an electronic solid-state system, which cannot be viewed as isolated, is rather challenging. Namely, it cannot be expected that the periodically driven system in contact with the heat bath (given among others by phonons) will simply form a band-insulating state with one band filled completely. Thus, one either has to resort to bath engineering in order to enforce a band insulating state [26,27] or explore novel opportunities of tailoring interesting system properties related to nonthermal occupations of (quasi)energy bands. In this section we do not address the issue of Floquet topological insulators, but present simple examples that show how the general formalism of Secs. II and III can be applied to compute nonequilibrium steady states of driven-dissipative Fermi gases.

In Fig. 15 we plot the mean occupations of a periodically driven tight-binding chain of $M=10$ states that is coupled to a heat bath and occupied by $N$ spinless (i.e., spin-polarized) noninteracting fermions. The state is trivial not only for zero filling $(N / M=0)$, but as a consequence of Pauli exclusion also for unit filling $(N / M=1)$, corresponding to zero filling of holes. For intermediate filling $N / M$ we find occupation numbers whose exact values [obtained from solving the manybody rate equation (13)] are well described by mean-field theory. Residual deviations of the mean-field theory are cured within the augmented mean-field theory (Sec. III C). 


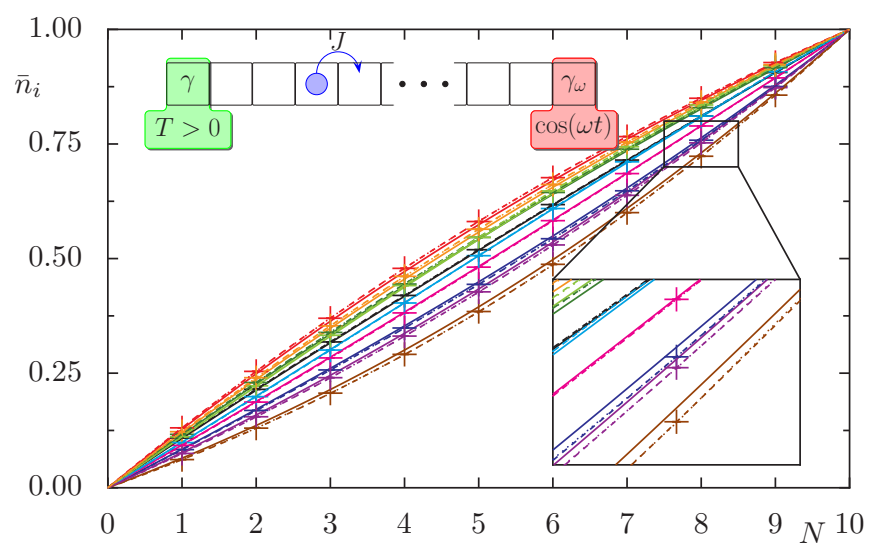

FIG. 15. (Color online) Mean occupations versus total number of fermions $N$ for a driven tight-binding chain with tunneling parameter $J$ and $M=10$ sites. The chain is coupled to a heat bath of temperature $T=J$ at the first site and it is driven away from equilibrium by a time-periodic potential modulation at the last site of frequency $\hbar \omega=$ $1.5 \mathrm{~J}$ and driving strength $\gamma_{\omega}=2.3$. Data obtained from mean-field theory (solid lines), augmented mean-field theory (dashed lines), and exact solution of the many-body rate equation (crosses).

As another example, we have computed steady states of a fermionic tight-binding chain of $M=100$ sites (see Sec. IID) and half filling $(N=M / 2)$. In Fig. 16 we plot the mean occupations of the single-particle states $i$ of the chain versus their energy $E_{i}=-2 J \cos \left(k_{i}\right)$, where $k_{i}$ is the wave number of state $i$. In panel (a) the equilibrium situation is shown, where the system is coupled to a single heat bath of intermediate temperature $T=J$. The nonequilibrium system coupled to two baths of different positive temperature $T_{1}=\mathrm{J}$ and $T_{2}=0.5 \mathrm{~J}$ shows qualitatively similar behavior, as can be seen from panel (b). In both situations (a) and (b) the occupations decrease with increasing energy. In striking contrast, the occupations depend in a nonmonotonous fashion on the energy, when the second heat bath is population inverted and described by a negative temperature. This can be seen in panels (c) and (d). Moreover, the distribution of occupations depends sensitively on the structure of the system-bath coupling. Depending on whether bath 1 is coupled to the first site [panel (c)] or to the third site [panel (d)] the occupation of the ground state assumes either a local minimum or a local maximum. Thus, like in the bosonic case, already the ideal Fermi gas offers many possibilities of dissipative state engineering far from equilibrium. Exploring these possibilities is, however, beyond the scope of the present paper.

\section{CONCLUSIONS AND OUTLOOK}

In this paper, we describe several aspects of nonequilibrium steady states of driven-dissipative ideal quantum gases. We focus on systems of sharp particle number that are driven away from equilibrium either by the coupling to two heat baths of different temperature or by time-periodic driving in combination with the coupling to a heat bath. We describe analytical and numerical methods for treating these systems within the framework of (Floquet-)Born-Markov theory and apply them both to bosonic and fermionic quantum gases.
On that basis, we work out a theory of Bose selection, a nonequilibrium generalization of Bose condensation, where multiple states can acquire large occupations. Also, the possibility of bath engineering in a fermionic lattice system is pointed out. Our results demonstrate that already ideal quantum gases give rise to intriguing and unexpected behavior, when they are driven into a steady state far from equilibrium. In the future it will be interesting to find applications for dissipative quantum engineering, e.g., in order to control the heat conductivity of a system in a robust fashion. On a theoretical level, it will be interesting to extend the formalism to systems exchanging particles with their environment and to include the effect of interactions.

\section{ACKNOWLEDGMENTS}

We thank Erwin Frey, Johannes Knebel, Alexander Leymann, Markus Weber, Jan Wiersig, and, in particular, Alexander Schnell for valuable discussions. Support through DFG Forschergruppe 760 "Scattering Systems with Complex Dynamics" is acknowledged. D.V. is grateful for the support from the Studienstiftung des Deutschen Volkes.

\section{APPENDIX A: MANY-BODY RATE EQUATION FROM LINDBLAD MASTER EQUATION}

Here we derive the equations of motion for the many-body occupation probabilities $p_{\boldsymbol{n}}=\langle\boldsymbol{n}|\rho| \boldsymbol{n}\rangle$, based on the Markovian master equation with the Liouvillian, Eq. (1). Replacing the single-particle operators $|i\rangle\langle j|$ with their representation in Fock space $\hat{a}_{i}^{\dagger} \hat{a}_{j}$ the equations of motion for the diagonal elements of the density operator take the form

$$
\begin{aligned}
\dot{p}_{\boldsymbol{n}}(t)= & \langle\boldsymbol{n}|\hat{\rho}(t)| \boldsymbol{n}\rangle \\
= & \sum_{i, j=1}^{M} \boldsymbol{R}_{i j}\left[\left\langle\boldsymbol{n}\left|\hat{a}_{i}^{\dagger} \hat{a}_{j} \hat{\rho}(t) \hat{a}_{j}^{\dagger} \hat{a}_{i}\right| \boldsymbol{n}\right\rangle\right. \\
& \left.-\frac{1}{2}\left\langle\boldsymbol{n}\left|\left\{\hat{\rho}(t), \hat{a}_{j}^{\dagger} \hat{a}_{i} \hat{a}_{i}^{\dagger} \hat{a}_{j}\right\}\right| \boldsymbol{n}\right\rangle\right] .
\end{aligned}
$$

For $i=j$ both terms inside the bracket cancel each other. For $i \neq j$, we have $\hat{a}_{j}^{\dagger} \hat{a}_{i}|\boldsymbol{n}\rangle=\sqrt{n_{i}\left(1 \pm n_{j}\right)}\left|\boldsymbol{n}_{j i}\right\rangle$ and $\hat{a}_{j}^{\dagger} \hat{a}_{i} \hat{a}_{i}^{\dagger} \hat{a}_{j}|\boldsymbol{n}\rangle=n_{j}\left(1 \pm n_{i}\right)|\boldsymbol{n}\rangle$, where the upper (lower) sign applies to bosons (fermions). Thus, the master equation simplifies to

$$
\begin{aligned}
\dot{p}_{\boldsymbol{n}}(t) & =\sum_{i, j=1}^{M} R_{i j}\left[n_{i}\left(1 \pm n_{j}\right) p_{\boldsymbol{n}_{j i}}(t)-n_{j}\left(1 \pm n_{i}\right) p_{\boldsymbol{n}}(t)\right] \\
& =\sum_{i, j=1}^{M}\left(1 \pm n_{j}\right) n_{i}\left[R_{i j} p_{\boldsymbol{n}_{j i}}(t)-R_{j i} p_{\boldsymbol{n}}(t)\right]
\end{aligned}
$$

wherein $\boldsymbol{n}_{j i}=\left(n_{1}, \ldots, n_{i}-1, \ldots, n_{j}+1, \ldots\right)$ denotes the occupation numbers obtained from $\boldsymbol{n}$ by transferring one particle from $i$ to $j$. We have not explicitly excluded the $i=j$ terms, since they still cancel. The second line was obtained by exchanging $i$ and $j$ in the second term. 

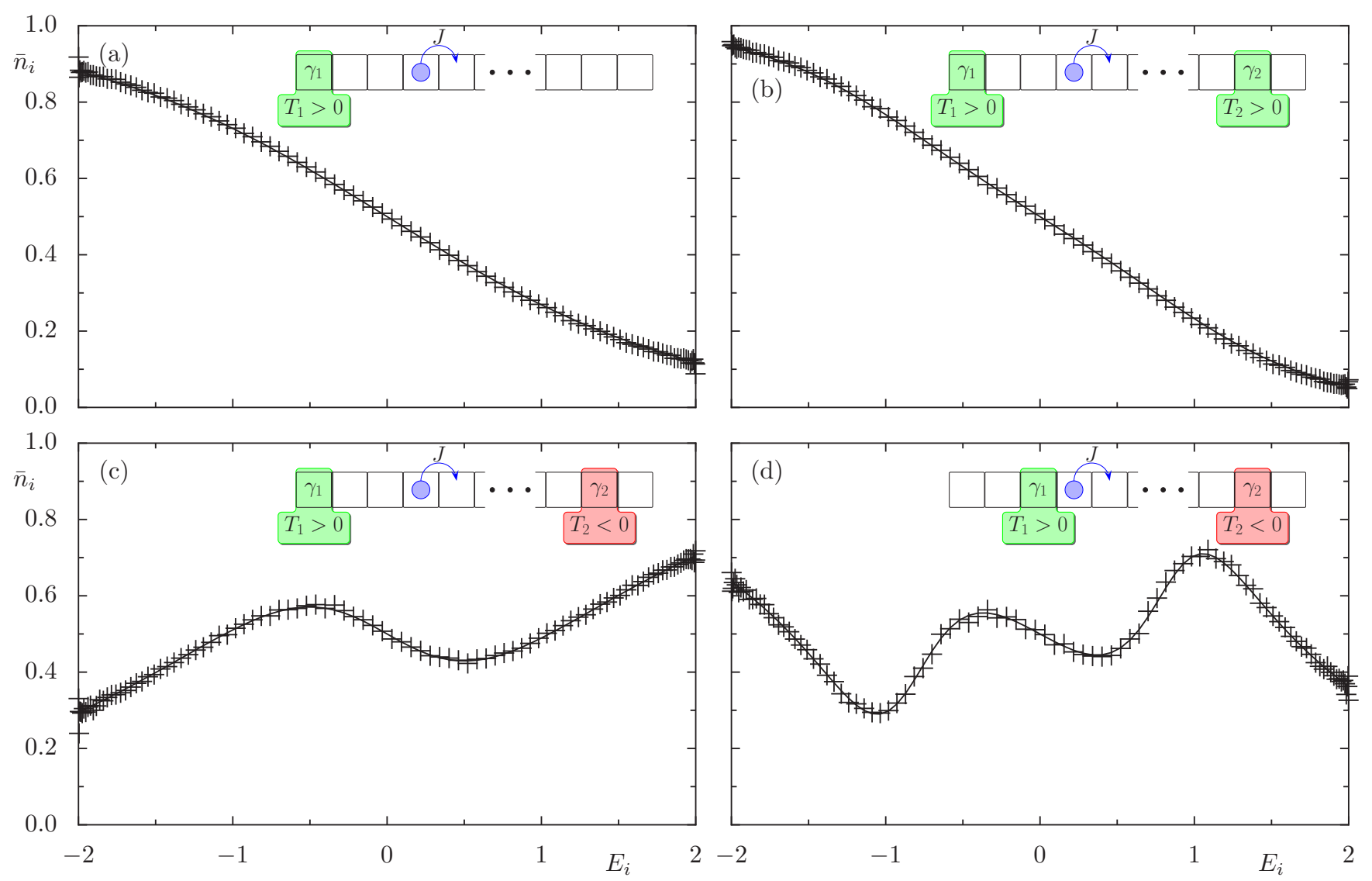

FIG. 16. (Color online) Mean-occupations of the single-particle energy eigenstates in a tight-binding chain of $M=100$ sites occupied by $N=M / 2$ spinless (i.e., spin-polarized) fermions versus the energy (in units of the tunneling parameter $J$ ). Data obtained from mean-field theory (solid lines) and exact Monte Carlo simulations (crosses). (a) Equilibrium situation where the chain is coupled to one bath of temperature $T=1 J$. (b) The chain is driven away from equilibrium by two heat baths of different positive temperature $\left(T_{1}=J\right.$ and $\left.T_{2}=0.5 \mathrm{~J}\right)$, coupled to the first and the next-to-last site with $\gamma_{1}=\gamma_{2}$. (c) Same as in (b), but now the second bath is population inverted and described by the negative temperature $T_{2}=-J$. (d) Like in (c), but now the first bath is coupled to the third site.

\section{APPENDIX B: EQUATIONS OF MOTION FOR MEAN OCCUPATIONS}

The equations of motion for the mean occupations read

$$
\begin{aligned}
\frac{d}{d t} \bar{n}_{k}(t)= & \operatorname{tr}\left[\hat{n}_{k} \frac{d}{d t} \hat{\rho}(t)\right]=\sum_{i, j} R_{i j} \operatorname{tr}\left[\hat{n}_{k} \hat{a}_{i}^{\dagger} \hat{a}_{j} \hat{\rho}(t) \hat{a}_{j}^{\dagger} \hat{a}_{i}\right. \\
& \left.-\frac{1}{2} \hat{n}_{k}\left\{\hat{a}_{j}^{\dagger} \hat{a}_{i} \hat{a}_{i}^{\dagger} \hat{a}_{j}, \hat{\rho}(t)\right\}\right],
\end{aligned}
$$

where we have employed Eq. (1) with the jump operators given by Eq. (12). The first term of the sum can be written like

$$
\begin{aligned}
\operatorname{tr}\left[\hat{n}_{k} \hat{a}_{i}^{\dagger} \hat{a}_{j} \hat{\rho}(t) \hat{a}_{j}^{\dagger} \hat{a}_{i}\right]= & \operatorname{tr}\left[\hat{n}_{k} \hat{a}_{j}^{\dagger} \hat{a}_{i} \hat{a}_{i}^{\dagger} \hat{a}_{j} \hat{\rho}(t)\right] \\
& +\left(\delta_{i k}-\delta_{j k}\right) \operatorname{tr}\left[\hat{a}_{j}^{\dagger} \hat{a}_{i} \hat{a}_{i}^{\dagger} \hat{a}_{j} \hat{\rho}(t)\right] .
\end{aligned}
$$

Here we have used the invariance of cyclic permutations under the trace as well as the relation

$$
\left[\hat{a}_{j}^{\dagger} \hat{a}_{i}, \hat{n}_{k}\right]=\hat{a}_{j}^{\dagger} \hat{a}_{i}\left(\delta_{i k}-\delta_{j k}\right)
$$

This relation is valid for particles of either statistics, as it can be obtained both by employing either the commutation relation $\left[\hat{a}_{i}, \hat{a}_{j}^{\dagger}\right]=\delta_{i j}$ for bosons or the anticommutation relation $\left\{\hat{a}_{i}, \hat{a}_{j}^{\dagger}\right\}=\delta_{i j}$ for fermions. We can now use

$$
\hat{a}_{j}^{\dagger} \hat{a}_{i} \hat{a}_{i}^{\dagger} \hat{a}_{j}=\hat{n}_{j}\left(1 \pm \hat{n}_{i}\right) \mp \delta_{i j} \hat{n}_{i},
$$

with the upper (lower) sign referring to bosons (fermions), to arrive at

$$
\begin{aligned}
\frac{d}{d t} \bar{n}_{k}(t)= & \sum_{i, j} R_{i j}\left(\delta_{i k}-\delta_{j k}\right) \operatorname{tr}\left[\hat{n}_{j}\left(1 \pm \hat{n}_{i}\right) \hat{\rho}(t)\right] \\
= & \sum_{j=1}^{M}\left\{R_{k j}\left[\bar{n}_{j}(t) \pm\left\langle\hat{n}_{k} \hat{n}_{j}\right\rangle(t)\right]\right. \\
& \left.-R_{j k}\left[\bar{n}_{k}(t) \pm\left\langle\hat{n}_{k} \hat{n}_{j}\right\rangle(t)\right]\right\} .
\end{aligned}
$$

\section{APPENDIX C: MEAN OCCUPATION AND CORRELATION IN PROJECTED GAUSSIAN STATE}

Calculating expectation values, like mean occupation or second-order correlations, for the projected Gaussian state,

$$
\hat{\rho}_{\text {proj }} \propto \hat{P}_{N} \hat{\rho}_{g} \hat{P}_{N},
$$

with

$$
\hat{P}_{N}=\sum_{\boldsymbol{n} \mid \sum_{i} \hat{n}_{i}=N}|\boldsymbol{n}\rangle\langle\boldsymbol{n}|,
$$


is a nontrivial problem. This is why, already in equilibrium it is typically much easier to treat a system in the grand-canonical rather than in the canonical ensemble. In this appendix we describe a method for computing expectation values

$$
\langle\hat{A}\rangle_{N}=\operatorname{tr}\left(\hat{A} \hat{\rho}_{\text {proj }}\right)=\frac{1}{Z} \sum_{n}^{N}\langle\boldsymbol{n}|\hat{A}| \boldsymbol{n}\rangle e^{-\sum_{k} \eta_{k} n_{k}}
$$

for projected Gaussian states numerically. Here the sum $\sum_{n}^{N}$ is constrained to Fock states of total particle number $N$ and $Z=\sum_{n}^{N} \exp \left(-\sum_{k} \eta_{k} n_{k}\right)$ denotes the partition function.

We focus on the mean occupations,

$$
\left\langle\hat{n}_{i}\right\rangle_{N}=\frac{1}{Z} \sum_{n}^{N} n_{i} e^{-\sum_{k} \eta_{k} n_{k}},
$$

and the two-particle correlations,

$$
\left\langle\hat{n}_{i} \hat{n}_{j}\right\rangle_{N}=\frac{1}{Z} \sum_{n}^{N} n_{i} n_{j} e^{-\sum_{k} \eta_{k} n_{k}} .
$$

The first expectation value can be written as

$$
\left\langle\hat{n}_{i}\right\rangle_{N}=\frac{1}{Z} \sum_{n_{i}} n_{i} e^{-\eta_{i} n_{i}} Z_{N-n_{i}}^{\backslash\{i\}}
$$

wherein

$$
Z_{N_{R}}^{\backslash_{R}}=\sum_{\left\{n_{k}\right\}_{k \notin S_{R}}}^{N_{R}} \exp \left(-\sum_{l \notin S_{R}} \eta_{l} n_{l}\right)
$$

is the partition function of fictitious system obtained by the original one by removing the states $S_{R}$ and filling it with $N_{R}$ particles only.

The second expectation value reads

$$
\left\langle\hat{n}_{i} \hat{n}_{j}\right\rangle_{N}=\frac{1}{Z} \sum_{n_{i}=0}^{N} \sum_{n_{j}=0}^{N-n_{i}} n_{i} n_{j} e^{-\eta_{i} n_{i}-\eta_{j} n_{j}} Z_{N-n_{i}-n_{j}}^{\backslash\{i, j\}} .
$$

The remaining partition functions can be calculated by exploiting the recursion formula [73]

$$
Z_{N}=\frac{1}{N} \sum_{k+1}^{N}( \pm 1)^{k+1} Z_{N-k}
$$

This enables the numerical treatment of systems with several thousands particles on $M=10$ states.

\section{APPENDIX D: EQUATIONS OF MOTION FOR TWO-PARTICLE CORRELATIONS}

In this appendix we derive the equations of motion for the two-particle correlations $\left\langle\hat{n}_{k} \hat{n}_{i}\right\rangle(t)$ [Eq. (42)] and rewrite this as equations of motion for the nontrivial correlations $\zeta_{k i}=\left\langle\hat{\zeta}_{k} \hat{\zeta}_{i}\right\rangle=\left\langle\hat{n}_{k} \hat{n}_{i}\right\rangle-\bar{n}_{k} \bar{n}_{i}$ [Eq. (46)]. Together with the equations of motion for the mean occupations $\bar{n}_{i}(t)$, Eqs. (45), they build the set of equation for the augmented mean-field theory described in Sec. III C. Hereby we close the hierarchy of equation by assuming the three-particle correlations to be trivial. For the sake of a simple notation we suppress the time argument in the following.

The exact equations of motion for $\left\langle\hat{n}_{k} \hat{n}_{i}\right\rangle$ are obtained from the many-body master equation in Lindblad form Eq. (1) by multiplying it by $\hat{n}_{k} \hat{n}_{i}$ from the left and taking the trace,

$$
\frac{d}{d t}\left\langle\hat{n}_{k} \hat{n}_{i}\right\rangle=\operatorname{tr}\left(\hat{n}_{k} \hat{n}_{i} \dot{\hat{\rho}}\right)=\sum_{j, l} R_{l j} \operatorname{tr}\left(\hat{n}_{k} \hat{n}_{i} \hat{a}_{l}^{\dagger} \hat{a}_{j} \hat{\rho} \hat{a}_{j}^{\dagger} \hat{a}_{l}-\frac{1}{2} \hat{n}_{k} \hat{n}_{i}\left\{\hat{\rho}, \hat{a}_{j}^{\dagger} \hat{a}_{l} \hat{a}_{l}^{\dagger} \hat{a}_{j}\right\}\right) .
$$

Invoking cyclic permutation under the trace and using Eq. (B3) we regroup the operators as

$$
\hat{a}_{j}^{\dagger} \hat{a}_{l} \hat{n}_{k} \hat{n}_{i}=\hat{n}_{k} \hat{n}_{i} \hat{a}_{j}^{\dagger} \hat{a}_{l}+\left[\left(\delta_{l i}-\delta_{j i}\right) \hat{n}_{k}+\left(\delta_{l k}-\delta_{j k}\right) \hat{n}_{i}+\left(\delta_{l i}-\delta_{j i}\right)\left(\delta_{l k}-\delta_{j k}\right)\right] \hat{a}_{j}^{\dagger} \hat{a}_{l} .
$$

Here the first term and the anticommutator in Eq. (D1) form a commutator, which vanishes under the $\operatorname{trace}, \operatorname{tr}\left(\rho\left[\hat{n}_{k} \hat{n}_{i}, \hat{a}_{j}^{\dagger} \hat{a}_{l} \hat{a}_{l}^{\dagger} \hat{a}_{j}\right]\right)=$ 0 . Applying also the operator relation Eq. (B4) we arrive at

$$
\frac{d}{d t}\left\langle\hat{n}_{k} \hat{n}_{i}\right\rangle=\sum_{j, l} R_{l j} \operatorname{tr}\left\{\left[\left(\delta_{l i}-\delta_{j i}\right) \hat{n}_{k}+\left(\delta_{l k}-\delta_{j k}\right) \hat{n}_{i}+\left(\delta_{l i}-\delta_{j i}\right)\left(\delta_{l k}-\delta_{j k}\right)\right]\left[\hat{n}_{j}\left(1 \pm \hat{n}_{l}\right) \mp \delta_{j l} \hat{n}_{j}\right] \hat{\rho}\right\} .
$$

The term $\delta_{j l} \hat{n}_{j}$ vanishes in combination with each of the $\delta$ prefactors, leaving

$$
\frac{d}{d t}\left\langle\hat{n}_{k} \hat{n}_{i}\right\rangle=\sum_{j, l} R_{l j}\left[\left(\delta_{l i}-\delta_{j i}\right)\left(\left\langle\hat{n}_{k} \hat{n}_{j}\right\rangle \pm\left\langle\hat{n}_{k} \hat{n}_{j} \hat{n}_{l}\right\rangle\right)+\left(\delta_{l k}-\delta_{j k}\right)\left(\left\langle\hat{n}_{i} \hat{n}_{j}\right\rangle \pm\left\langle\hat{n}_{i} \hat{n}_{j} \hat{n}_{l}\right\rangle\right)+\left(\delta_{l i}-\delta_{j i}\right)\left(\delta_{l k}-\delta_{j k}\right)\left(\bar{n}_{j} \pm\left\langle\hat{n}_{j} \hat{n}_{l}\right\rangle\right)\right]
$$

Evaluating one of the two sums, we arrive at

$$
\begin{aligned}
\frac{d}{d t}\left\langle\hat{n}_{k} \hat{n}_{i}\right\rangle= & \pm \sum_{j}\left(A_{k j}+A_{i j}\right)\left\langle\hat{n}_{k} \hat{n}_{i} \hat{n}_{j}\right\rangle+\sum_{j}\left[R_{k j}\left\langle\hat{n}_{i} \hat{n}_{j}\right\rangle-R_{j k}\left\langle\hat{n}_{i} \hat{n}_{k}\right\rangle+R_{i j}\left\langle\hat{n}_{k} \hat{n}_{j}\right\rangle-R_{j i}\left\langle\hat{n}_{k} \hat{n}_{i}\right\rangle\right] \\
& +\delta_{i k} \sum_{j}\left[R_{k j}\left(\bar{n}_{j} \pm\left\langle\hat{n}_{j} \hat{n}_{k}\right\rangle\right)+R_{j k}\left(\bar{n}_{k} \pm\left\langle\hat{n}_{k} \hat{n}_{j}\right\rangle\right)\right]-R_{i k}\left(\bar{n}_{k} \pm\left\langle\hat{n}_{k} \hat{n}_{i}\right\rangle\right)-R_{k i}\left(\bar{n}_{i} \pm\left\langle\hat{n}_{i} \hat{n}_{k}\right\rangle\right)
\end{aligned}
$$

which is identical to Eq. (42). We separate the number operators $\hat{n}_{k}$ into their mean part $\bar{n}_{k}$ and their fluctuations $\hat{\zeta}_{k}=\bar{n}_{k}-\hat{n}_{k}$. With that, the correlations read $\left\langle\hat{n}_{k} \hat{n}_{i}\right\rangle=\bar{n}_{k} \bar{n}_{i}+\zeta_{k i}$ with the nontrivial correlation $\zeta_{k i}=\left\langle\hat{\zeta}_{k} \hat{\zeta}_{i}\right\rangle$ and $\left\langle\hat{n}_{k} \hat{n}_{i} \hat{n}_{j}\right\rangle=\left\langle\hat{\zeta}_{k} \hat{\zeta}_{i} \hat{\zeta}_{j}\right\rangle+\bar{n}_{k} \zeta_{i j}+$ 
$\bar{n}_{i} \zeta_{k j}+\bar{n}_{j} \zeta_{k i}+\bar{n}_{k} \bar{n}_{i} \bar{n}_{j}$. Now Eq. (D5) can be rewritten as

$$
\begin{aligned}
\frac{d}{d t}\left\langle\hat{n}_{k} \hat{n}_{i}\right\rangle= & \pm \sum_{j}\left(A_{k j}+A_{i j}\right)\left[\left\langle\hat{\zeta}_{i} \hat{\zeta}_{k} \hat{\zeta}_{j}\right\rangle+\bar{n}_{i} \zeta_{k j}+\bar{n}_{k} \zeta_{i j}+\bar{n}_{j} \zeta_{i k}+\bar{n}_{k} \bar{n}_{i} \bar{n}_{j}\right] \\
& +\sum_{j}\left(R_{k j}\left[\bar{n}_{i} \bar{n}_{j}+\zeta_{i j}\right]-R_{j i}\left[\bar{n}_{i} \bar{n}_{k}+\zeta_{i k}\right]+R_{i j}\left[\bar{n}_{k} \bar{n}_{j}+\zeta_{k j}\right]-R_{j i}\left[\bar{n}_{k} \bar{n}_{i}+\zeta_{k i}\right]\right) \\
& +\delta_{k i} \sum_{j}\left\{ \pm\left(R_{j k}+R_{k j}\right)\left[\bar{n}_{j} \bar{n}_{k}+\zeta_{j k}\right]+\left(R_{k j} \bar{n}_{j}+R_{j k} \bar{n}_{k}\right)\right\} \mp\left(R_{k i}+R_{i k}\right)\left[\bar{n}_{k} \bar{n}_{i}+\zeta_{k i}\right]-\left(R_{i k} \bar{n}_{k}+R_{k i} \bar{n}_{i}\right)
\end{aligned}
$$

To obtain the equations of motion for the nontrivial correlations $\zeta_{k i}$, we subtract

$$
\frac{d}{d t}\left(\bar{n}_{k} \bar{n}_{i}\right)=\bar{n}_{k} \sum_{j}\left\{R_{i j}\left[\bar{n}_{j}\left(1 \pm \bar{n}_{i}\right) \pm \zeta_{i j}\right]-R_{j i}\left[\bar{n}_{i}\left(1 \pm \bar{n}_{j}\right) \pm \zeta_{i j}\right]\right\}+\bar{n}_{i} \sum_{j}\left\{R_{k j}\left[\bar{n}_{j}\left(1 \pm \bar{n}_{k}\right) \pm \zeta_{k j}\right]-R_{j k}\left[\bar{n}_{k}\left(1 \pm \bar{n}_{j}\right) \pm \zeta_{k j}\right]\right\}
$$

from Eq. (D6) to obtain

$$
\begin{aligned}
\frac{d \zeta_{k i}}{d t}= & \pm \sum_{j}\left[\left(A_{k j}+A_{i j}\right)\left(\left\langle\hat{\zeta}_{i} \hat{\zeta}_{k} \hat{\zeta}_{j}\right\rangle+\bar{n}_{j} \zeta_{i k}\right)+A_{k j} \bar{n}_{k} \zeta_{i j}+A_{i j} \bar{n}_{i} \zeta_{k j}\right]+\sum_{j}\left[R_{k j} \zeta_{j i}-R_{j k} \zeta_{k i}+R_{i j} \zeta_{k j}-R_{j i} \zeta_{k i}\right] \\
& +\delta_{k i} \sum_{j}\left[ \pm\left(R_{j k}+R_{k j}\right)\left(\bar{n}_{j} \bar{n}_{k}+\zeta_{j k}\right)+\left(R_{k j} \bar{n}_{j}+R_{j k} \bar{n}_{k}\right)\right] \mp\left(R_{k i}+R_{i k}\right)\left(\bar{n}_{k} \bar{n}_{i}+\zeta_{k i}\right)-\left(R_{i k} \bar{n}_{k}+R_{k i} \bar{n}_{i}\right) .
\end{aligned}
$$

Finally, neglecting nontrivial three-particle correlations, $\left\langle\hat{\zeta}_{k} \hat{\zeta}_{i} \hat{\zeta}_{j}\right\rangle \approx 0$, one arrives at the nonlinear set of equations (46), which defines together with Eqs. (45) the augmented mean-field theory.

\section{APPENDIX E: PARAMETER-DEPENDENT SOLUTION OF THE AUXILIARY MATRIX $\tilde{A}(p)$}

For the auxiliary rate-asymmetry matrix $\tilde{A}(p)$ given by Eqs. (87) and (88), the problem (80) takes the form

$$
\begin{aligned}
\tilde{\mu}_{i}(p)= & \sum_{j}\left(A_{i j}+p B_{i j}\right) \tilde{v}_{j}(p), \\
& \text { with }\left\{\begin{array}{l}
\tilde{v}_{i}>0 \text { and } \tilde{\mu}_{i}=0 \text { for } i \in \tilde{\mathcal{S}}(p), \\
\tilde{v}_{i}=0 \text { and } \tilde{\mu}_{i}<0 \text { for } i \notin \tilde{\mathcal{S}}(p) .
\end{array}\right.
\end{aligned}
$$

Together with Eq. (88) restricting $B$ to have a crosslike structure, this implies

$$
\sum_{j \in \tilde{\mathcal{S}}(p)}\left(A_{i j}+p \delta_{i k} b_{j}-p \delta_{k j} b_{i}\right) \tilde{v}_{j}(p)=0, \quad i \in \tilde{\mathcal{S}}(p)
$$

Let us now show that, unless a transition occurs where the set of selected states $\tilde{\mathcal{S}}(p)$ changes, the solution $\tilde{v}(p)$ varies, apart from a normalization factor, linearly with $p$ as written in Eq. (91).

For that purpose we decompose the solution $\tilde{v}_{i}(p)$ like

$$
\tilde{v}_{i}(p)=\tilde{v}_{i}^{(0)}+\Delta \tilde{\nu}_{i}(p), \quad i \in \tilde{\mathcal{S}}(p),
$$

where $\tilde{v}_{i}^{(0)}$ is defined to solve

$$
\sum_{i \in \tilde{\mathcal{S}}(p)} A_{i j} \tilde{v}_{i}^{(0)}=0, \quad i \in \tilde{\mathcal{S}}(p) .
$$

These equations possess a solution, since $A_{i j}$ is a skewsymmetric matrix acting in the odd-dimensional subspace spanned by the selected states. However, the $\tilde{v}_{i}^{(0)}$ can be negative, as $\tilde{\mathcal{S}}(p)$ contains the selected states for the matrix $\tilde{A}(p)$ and not for $A$.
We can now distinguish two cases. If the state $k$ is not contained in the set of selected states, $k \notin \tilde{\mathcal{S}}(k)$, Eqs. (E2) simply reduce to Eq. (E4), so that we find the trivial parameter dependence,

$$
\tilde{v}_{i}(p)=\tilde{v}_{i}^{(0)} \quad i \in \tilde{\mathcal{S}}(p),
$$

which complies with Eq. (91). If the state $k$ is contained in the set of selected states, $k \in \tilde{\mathcal{S}}(k)$, it is convenient to discard the normalization condition $\sum_{i \in \tilde{\mathcal{S}}(p)} \tilde{v}_{i}(p)=1$ for the moment, in favor of requiring

$$
\tilde{v}_{k}(p)=\tilde{v}_{k}^{(0)}
$$

i.e.,

$$
\Delta \tilde{\nu}_{k}(p)=0 .
$$

Note that this requires also to fix the solution of the homogeneous equations (E4) such that $\tilde{v}_{k}^{(0)}>0$, which we can always do. With that, all other states in $\tilde{\mathcal{S}}(p)$ obey

$$
\sum_{j \in \tilde{\mathcal{S}}(p) \backslash\{k\}} A_{i j} \Delta \tilde{\nu}_{j}(p)=p \tilde{v}_{k}^{(0)} b_{i}, \quad i \in \tilde{\mathcal{S}}(p) \backslash\{k\} .
$$

This set of inhomogeneous equations possesses a solution, since $A_{i j}$ is a skew-symmetric matrix acting in the evendimensional subspace spanned by the states of $\tilde{\mathcal{S}}(p) \backslash\{k\}$, which has no eigenvalue zero without fine tuning. The solution $\Delta \tilde{v}_{j}(p)$ will depend linearly on the parameter $p$. Therefore, one finds that the $\tilde{v}_{i}(p)$ depend linearly on the parameter $p$,

$$
\tilde{v}_{i}(p)=\tilde{v}_{i}^{(0)}+c_{i} p, \quad i \in \tilde{\mathcal{S}}(p) .
$$

In order to restore the normalization condition $\sum_{i \in \tilde{\mathcal{S}}(p)} \tilde{v}_{i}(p)=$ 1 , we can now redefine

$$
\tilde{v}_{i}(p)=\tilde{C}(p)\left[\tilde{v}_{i}^{(0)}+c_{i} p\right], \quad i \in \tilde{\mathcal{S}}(p),
$$


with normalization constant $\tilde{C}(p)>0$. One finds

$$
\tilde{C}^{-1}(p)=\sum_{i \in \tilde{\mathcal{S}}(p)}\left[\tilde{v}_{i}^{(0)}+c_{i} p\right]=1+p \sum_{i \in \tilde{\mathcal{S}}(p)} c_{i},
$$

where the second equality holds if we choose $\sum_{i \in \tilde{\mathcal{S}}(p)} \tilde{v}_{i}^{(0)}=1$, which we always can. Equation (E10) implies that Eq. (91) is fulfilled also if $k \in \tilde{\mathcal{S}}(k)$.
[1] M. Campisi, P. Hänggi, and P. Talkner, Rev. Mod. Phys. 83, 771 (2011).

[2] J. Dziarmaga, Adv. Phys. 59, 1063 (2010).

[3] A. Polkovnikov, K. Sengupta, A. Silva, and M. Vengalattore, Rev. Mod. Phys. 83, 863 (2011).

[4] D. M. Basko, I. L. Aleiner, and B. L. Altshuler, Ann. Phys. 321, 1126 (2006).

[5] D. A. Huse, R. Nandkishore, V. Oganesyan, A. Pal, and S. L. Sondhi, Phys. Rev. B 88, 014206 (2013).

[6] A. Eckardt, C. Weiss, and M. Holthaus, Phys. Rev. Lett. 95, 260404 (2005).

[7] T. Oka and H. Aoki, Phys. Rev. B 79, 081406 (2009).

[8] A. Zenesini, H. Lignier, D. Ciampini, O. Morsch, and E. Arimondo, Phys. Rev. Lett. 102, 100403 (2009).

[9] J. Struck, C. Ölschläger, R. Le Targat, P. Soltan-Panahi, A. Eckardt, M. Lewenstein, P. Windpassinger, and K. Sengstock, Science 333, 996 (2011).

[10] G. Jotzu, M. Messer, T. U. Rémi Desbuquois, Martin Lebrat, D. Greif, and T. Esslinger, Nature (London) 515, 237 (2014).

[11] M. Aidelsburger, M. Lohse, C. Schweizer, M. Atala, J. T. Barreiro, S. Nascimbène, N. R. Cooper, I. Bloch, and N. Goldman, Nat. Phys. 11, 162 (2015).

[12] D. Fausti, R. I. Tobey, N. Dean, S. Kaiser, A. Dienst, M. C. Hoffmann, S. Pyon, T. Takayama, H. Takagi, and A. Cavalleri, Science 331, 189 (2011).

[13] S. Lepri, R. Livi, and A. Politi, Phys. Rep. 377, 1 (2003).

[14] R. K. P. Zia and B. Schmittmann, J. Stat. Mech. (2007) P07012.

[15] Y. Dubi and M. Di Ventra, Rev. Mod. Phys. 83, 131 (2011).

[16] N. A. Zimbovskaya and M. R. Pederson, Phys. Rep. 509, 1 (2011).

[17] T. Prosen, Phys. Rev. Lett. 107, 137201 (2011).

[18] N. Li, J. Ren, L. Wang, G. Zhang, P. Hänggi, and B. Li, Rev. Mod. Phys. 84, 1045 (2012).

[19] M. Müller, S. Diehl, G. Pupillo, and P. Zoller, Adv. At. Mol. Opt. Phys. 61, 1 (2012).

[20] A. Dhar, K. Saito, and P. Hänggi, Phys. Rev. E 85, 011126 (2012).

[21] E. Lutz and F. Renzoni, Nat. Phys. 9, 615 (2013).

[22] T. Prosen, Phys. Rev. Lett. 112, 030603 (2014).

[23] D. Vorberg, W. Wustmann, R. Ketzmerick, and A. Eckardt, Phys. Rev. Lett. 111, 240405 (2013).

[24] T. Shirai, T. Mori, and S. Miyashita, Phys. Rev. E 91, 030101 (2015).

[25] C. Chen, J.-H. An, H.-G. Luo, C. P. Sun, and C. H. Oh, Phys. Rev. A 91, 052122 (2015).

[26] T. Iadecola, T. Neupert, and C. Chamon, Phys. Rev. B 91, 235133 (2015).

[27] K. I. Seetharam, C.-E. Bardyn, N. H. Lindner, M. S. Rudner, and R. Gil, arXiv:1502.02664.

[28] M. J. Hartmann, F. G. Brandao, and M. B. Plenio, Laser Photon. Rev. 2, 527 (2008).
[29] R. J. Schoelkopf and S. M. Girvin, Nature (London) 451, 664 (2008).

[30] J. Klaers, J. Schmitt, F. Vewinger, and M. Weitz, Nature (London) 468, 545 (2010).

[31] A. A. Houk, H. E. Türeci, and J. Koch, Nat. Phys. 8, 292 (2012).

[32] I. Carusotto and C. Ciuti, Rev. Mod. Phys. 85, 299 (2013).

[33] T. Byrnes, N. Y. Kim, and Y. Yamamoto, Nat. Phys. 10, 803 (2014).

[34] G. Bianconi and A.-L. Barabási, Phys. Rev. Lett. 86, 5632 (2001).

[35] Z. Burda, D. Johnston, J. Jurkiewicz, M. Kaminski, M. A. Nowak, G. Papp, and I. Zahed, Phys. Rev. E 65, 026102 (2002).

[36] D. Chowdhury, L. Santen, and A. Schadschneider, Phys. Rep. 329, 199 (2000).

[37] M. R. Evans and T. Hanney, J. Phys. A: Math. Gen. 38, R195 (2005).

[38] M. R. Evans, T. Hanney, and S. N. Majumdar, Phys. Rev. Lett. 97, 010602 (2006).

[39] Y. Schwarzkopf, M. R. Evans, and D. Mukamel, J. Phys. A 41, 205001 (2008).

[40] O. Hirschberg, D. Mukamel, and G. M. Schütz, Phys. Rev. Lett. 103, 090602 (2009).

[41] S.-W. Kim, J. Lee, and J. D. Noh, Phys. Rev. E 81, 051120 (2010).

[42] A. G. Thompson, J. Tailleur, M. E. Cates, and R. A. Blythe, J. Stat. Mech. (2010) P02013.

[43] A. Schadschneider, D. Chowdhury, and K. Nishinari, Stochastic Transport in Complex Systems: From Molecules to Vehicles (Elsevier, Amsterdam, 2010).

[44] S. Grosskinsky, F. Redig, and K. Vafayi, J. Stat. Phys. 142, 952 (2011).

[45] N. van Kampen, Stochastic Processes in Physics and Chemistry, 3rd ed. (Elsevier, Amsterdam, 2007).

[46] J. Knebel, T. Krüger, M. F. Weber, and E. Frey, Phys. Rev. Lett. 110, 168106 (2013).

[47] J. Knebel, M. F. Weber, T. Krüger, and E. Frey, Nat. Commun. 6, 6977 (2015).

[48] H. Breuer and F. Petruccione, The Theory of Open Quantum Systems (Oxford University Press, Oxford \& New York, 2002).

[49] R. Blümel, A. Buchleitner, R. Graham, L. Sirko, U. Smilansky, and H. Walther, Phys. Rev. A 44, 4521 (1991).

[50] S. Kohler, T. Dittrich, and P. Hänggi, Phys. Rev. E 55, 300 (1997).

[51] H.-P. Breuer, W. Huber, and F. Petruccione, Phys. Rev. E 61, 4883 (2000).

[52] D. W. Hone, R. Ketzmerick, and W. Kohn, Phys. Rev. E 79, 051129 (2009).

[53] N. H. Lindner, G. Refael, and V. Galitzki, Nat. Phys. 7, 490 (2012).

[54] J. H. Shirley, Phys. Rev. 138, B979 (1965).

[55] Y. B. Zel'dovich, J. Exp. Theor. Phys. 24, 1006 (1967). 
[56] H. Sambe, Phys. Rev. A 7, 2203 (1973).

[57] J. Schnakenberg, Rev. Mod. Phys. 48, 571 (1976).

[58] M. Langemeyer and M. Holthaus, Phys. Rev. E 89, 012101 (2014).

[59] W. Wustmann, Ph.D. thesis, Technische Universität Dresden, 2010.

[60] R. Ketzmerick and W. Wustmann, Phys. Rev. E 82, 021114 (2010).

[61] M. B. Plenio and P. L. Knight, Rev. Mod. Phys. 70, 101 (1998).

[62] K. Mølmer and Y. Castin, Quantum Opt. 8, 49 (1996).

[63] D. T. Gillespie, J. Comput. Phys. 22, 403 (1976).

[64] A. Gelman and D. B. Rubin, Stat. Sci. 7, 457 (1992).

[65] Y. Castin, J. Phys. IV (France) 116, 89 (2004).

[66] M. Holthaus, E. Kalinowski, and K. Kirsten, Ann. Phys. 270, 198 (1998).
[67] R. K. Pathria, Statistical Mechanics (Pergamon Press, Oxford, 1972).

[68] O. Penrose and L. Onsager, Phys. Rev. 104, 576 (1956).

[69] T. Chawanya and K. Tokita, J. Phys. Soc. Jpn. 71, 429 (2002).

[70] N. N. Bogoliubov, J. Phys. (USSR) 11, 23 (1947), reprinted in D. Pines, The Many-Body Problem (W. A. Benjamin, New York, 1961), p. 292.

[71] A. R. Kolovsky, Europhys. Lett. 93, 20003 (2011).

[72] P. Hauke, O. Tieleman, A. Celi, C. Ölschläger, J. Simonet, J. Struck, M. Weinberg, P. Windpassinger, K. Sengstock, M. Lewenstein, and A. Eckardt, Phys. Rev. Lett. 109, 145301 (2012).

[73] P. Borrmann and G. Franke, J. Chem. Phys. 98, 2484 (1993). 